\title{
Mobilizing prior knowledge in text processing : the selective-attention hypothesis versus the cognitive set-point hypothesis
}

Citation for published version (APA):

Machiels-Bongaerts, M. I. A. (1993). Mobilizing prior knowledge in text processing : the selective-attention hypothesis versus the cognitive set-point hypothesis. [Doctoral Thesis, Maastricht University]. Datawyse / Universitaire Pers Maastricht. https://doi.org/10.26481/dis.19931222mm

Document status and date:

Published: 01/01/1993

DOI:

10.26481/dis.19931222mm

Document Version:

Publisher's PDF, also known as Version of record

Please check the document version of this publication:

- A submitted manuscript is the version of the article upon submission and before peer-review. There can be important differences between the submitted version and the official published version of record.

People interested in the research are advised to contact the author for the final version of the publication, or visit the DOI to the publisher's website.

- The final author version and the galley proof are versions of the publication after peer review.

- The final published version features the final layout of the paper including the volume, issue and page numbers.

Link to publication

\footnotetext{
General rights rights.

- You may freely distribute the URL identifying the publication in the public portal. please follow below link for the End User Agreement:

www.umlib.nl/taverne-license

Take down policy

If you believe that this document breaches copyright please contact us at:

repository@maastrichtuniversity.nl

providing details and we will investigate your claim.
}

Copyright and moral rights for the publications made accessible in the public portal are retained by the authors and/or other copyright owners and it is a condition of accessing publications that users recognise and abide by the legal requirements associated with these

- Users may download and print one copy of any publication from the public portal for the purpose of private study or research.

- You may not further distribute the material or use it for any profit-making activity or commercial gain

If the publication is distributed under the terms of Article 25fa of the Dutch Copyright Act, indicated by the "Taverne" license above, 


\section{MOBILIZING PRIOR KNOWLEDGE IN TEXT PROCESSING}





\section{MOBILIZING PRIOR KNOWLEDGE IN TEXT PROCESSING}

\section{THE SELECTIVE-ATTENTION HYPOTHESIS VERSUS THE COGNITIVE SET-POINT HYPOTHESIS}

\section{PROEFSCHRIFT}

ter verkrijging van de graad van doctor aan de Rijksuniversiteit Limburg te Maastricht, op gezag van de Rector Magnificus, Prof. dr. H. Philipsen, volgens het besluit van het College van Dekanen, in het openbaar te verdedigen op woensdag,

22 december 1993 om 14.00 uur

door

Maureen Machiels-Bongaerts

geboren te Echt in 1962

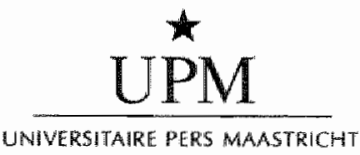




\section{PROMOTOR}

Prof. dr. H.G. Schmidt

\section{CO-PROMOTOR}

Dr. H.P.A. Boshuizen

\section{BEOORDELINGSCOMMISSIE}

Prof. dr. M.A. van den Hout (voorzitter)

Prof. dr. J. Jolles

Dr. A.J. Landeweend

Dr. I. Peeck

Dr. W. Vonk

Productie: Datawyse / Universitaire Pers Maastricht

CIP-DATA KONINKLI]KE BIBLIOTHEEK, DEN HAAG

Machiels-Bongaerts, Maureen

Mobilizing prior knowledge in text processing: the selective-attention hypothesis versus the cognitive set-point hypothesis / Maureen Machiels-Bongaerts. Maastricht: Universitaire Pers Maastricht. - III. Thesis Maastricht - With ref. - With summary in Dutch. ISBN 90-5278-108-7

Subject headings: prior knowledge / text processing. 
To my guys, Marcel and Bas 


\section{TABLE OF CONTENTS}

Preface

$x \mathrm{i}$

Chapter I The Impact of Prior Knowledge on Information Processing 1

Introduction

The advance organizer studies

The context effect studies

The role of domain knowledge

Perspective assignment

Prior knowledge mobilization

The selective-attention versus the cognitive set-point hypothesis

Divergent information processes

Research goals

Chapter II Investigating Two Conflicting Hypotheses: Pilot Study

Introduction

Pilot study

General discussion

Chapter III Effects of Prior Knowledge Activation on Study Time Allocation and Free Recall

Abstract

Introduction

Experiment 1

Experiment 2

Experiment 3 
Chapter IV Effects of Instructional Variation on Selective Attention 65

Introduction 65

Experiment 1 66

Experiment 2 71

Experiment $3 \quad 76$

$\begin{array}{ll}\text { General discussion } & 79\end{array}$

Chapter V Effects of Extending Relevant Information in Text on Selective Attention $\quad 81$

$\begin{array}{lr}\text { Introduction } & 81\end{array}$

Experiment $\quad 83$

General discussion $\quad 86$

Chapter VI Mobilizing Category Names: Studies on Free Recall and Study Time Allocation $\quad 89$

Abstract

Introduction

Experiment 1

Experiment 2

Experiment 3

General discussion

Chapter VII Summary and Implications

Introduction

Discussion and conclusions

Suggestions for further research

Educational implications

Samenvatting en Implicaties

References

Appendices

Curriculum Vitae 
PREFACE

Writing a thesis requires a huge amount of (cognitive) effort of a lot of people aside from the author whose name is printed on the cover. Here I want to express my gratitude to a number of colleagues, friends and other persons directly or indirectly involved in the process of finishing this thesis.

First, I would like to thank my "tutors", Henk Schmidt and Els Boshuizen, my promotor and co-promotor, who guided me through this process. Their suggestions, advice and corrections were very valuable to me. Despite their full schedules, there was always time and space to evaluate the progress of this thesis, albeit in the train to Amsterdam.

Conducting the experiments reported in this thesis would have been impossible without the help of Bart Reute, who constructed the computer programs for the study phases. Indispensable was the contribution of Taco Bisscheroux and Jacqueline Bongaerts, who both participated in the research reported as second raters of the recall protocols. Furthermore, I appreciated Gerard van Breukelen's advice with respect to the analyses conducted.

The materials applied in the studies presented could be construed thanks to information provided by the Dutch Ministry of Agriculture and Fisheries and the Portuguese Embassy, both located in The Hague. Furthermore, I would like to thank Jeannet Kalk for correcting the translation of the original Dutch text.

I would like to thank the Department of Educational Development and Research for providing the facilities to carry out the experiments. In this respect, Frans Ronteltap's contribution should be explicitly mentioned. In addition, Eugène Custers and Herco Fonteijn deserve credits for their comments on earlier versions of Chapter III and Petry Thiemann for checking the reference list. A special word of thank is addressed to Diana Dolmans, who was my room-mate at the Department for a number of years. She was always willing to discuss problems risen during the early phases of this thesis. 
Furthermore, the students who voluntarily participated in the studies reported ought to be thanked; without them, no data would have been available. This also refers to the Institute for Educational Research in the Netherlands (SVO); the studies described in this dissertation were enabled by grant $\# 7000$ from this institute.

Last but certainly not least I would like to thank Marcel and Bas for giving me the opportunity to "finish the job", although at the expense of many family hours. They were my home base to which I always could return to let off steam and relax. My solemn promise to them: I'll make it up to you. In addition, I would like to express my gratitude to my parents and in-laws who, aside from Marcel, took care of Bas, which allowed me to finish this thesis.

Maastricht-Echt, November 1993 
CHAPTER I

\section{THE IMPACT OF PRIOR KNOWLEDGE ON INFORMATION PROCESSING}

INTRODUCTION

Spilich, Vesonder, Chiesi and Voss (1979) presented two groups of subjects with a text about baseball on audiotape. The first subject group, the highknowledge group, consisted of experts in this domain, whereas the second group, the low-knowledge group, had no or little knowledge of baseball. After listening to the story, the subjects were asked to write down everything they could recall from the text. Subsequently, they were given a booklet containing a number of completion questions referring to the text. High-knowledge subjects recalled more of the text than low-knowledge subjects, specifically of information that was relevant to the topic, baseball. The two groups hardly differed in recall of information that was less relevant to the topic. The questionnaire provided similar results.

From this research as well as a number of other studies carried out in the last twenty years, it can be concluded that prior knowledge activation has a strong impact on information processing. Over the years, several hypotheses have been formulated trying to account for the effects of prior knowledge on information processing and recall. These hypotheses are quite divergent, probably because they were based on experiments that differ in the operationalization of prior knowledge.

In the next paragraphs of this chapter the evolution of psychological theory on the effects of prior knowledge on information processing will be chronologically described. From this historical review a transition will be 
made to the goals of the research presented in the next chapters of this dissertation.

\section{THE ADVANCE ORGANIZER STUDIES}

During the 1960s, the effects of prior knowledge activation were studied by means of so-called advance organizers (Ausubel, 1960, 1963, 1968). An advance organizer can be viewed as a learning aid to process and integrate new information in existing knowledge structures. The new study material is preceded by an advance organizer, containing familiar concepts from which the text can be understood at a more general, abstract level. The function of an advance organizer is "... to provide ideational scaffolding for the stable incorporation and retention of the more detailed and differentiated material that follows" (Ausubel, 1968, p. 148).

Ausubel and Youssef (1963) showed that information processing and recall can be facilitated by advance organizers. Students read a 2500 -words text about Buddhism. Prior to studying this text one group read an advance organizer, emphasizing the relationship between Buddhism and Christianity. The second group was provided with a historical introduction to the topic; this introduction could not be used as a framework from which the study text could be interpreted. Recall scores of the advance organizer group exceeded those of the second group. The advance organizer group was able to understand new concepts of Buddhism in light of Christianity terms. New information could be related to existing knowledge since these subjects had the disposal of "relevant proximate subsumers" (Ausubel, 1968, p. 148). Thus, according to Ausubel, the effects of advance organizers on information processing should be located at encoding. The advance organizer would influence the way in which new information is encoded in memory.

This encoding hypothesis, which is diametrically opposed to the socalled retrieval hypothesis, was challenged by Mayer and Bromage (1980). The retrieval hypothesis predicts facilitation effects of an advance organizer during information retrieval. According to Mayer and Bromage, the advance organizer may function as a recall aid. In order to resolve this dilemma, they presented two groups of subjects with a text about a new computer programming language. An advance organizer was presented to the first group before reading the text, whereas the second group did not receive the advance organizer until they had finished studying the text. After the study phase a recall test was carried out. The two subject groups appeared to differ in the type of information correctly recalled. The subjects who received an advance organizer prior to studying the text recalled more concepts and made more new inferences. The subjects who were provided with an advance organizer after studying the text exceeded in recall of technical ideas and vague sum- 
maries. From these findings, Mayer and Bromage concluded that the impact of advance organizers on recall is a strict encoding phenomenon. In other words, as a result of presenting an advance organizer prior to the study phase new information is processed, stored and retained in a different way. The retrieval hypothesis was rejected by Mayer and Bromage since differences in recall were found between two subject groups that strictly had the disposal of the same information: Both the study text and the advance organizer.

Mayer and Bromage (1980) considered these results as support for the assimilation encoding theory, which is related to Ausubel's (1968) subsumption theory. According to the assimilation encoding theory, the presentation of an advance organizer results in activating relevant prior knowledge which facilitates subsequent information processing. Due to an advance organizer new information can be integrated into the network of existing knowledge and experiences, resulting in restructuring the initial knowledge base.

Further support for the encoding hypothesis stems from Lambiotte and Dansereau (1990), who applied a different type of advance organizer that was presented during the study phase. Subjects whose knowledge of biology was extended and subjects with little knowledge in this particular domain attended two lectures that each lasted for about 20 minutes. Each of these subject groups was subdivided into three groups, resulting in six subgroups. One low-knowledge subgroup and one high-knowledge subgroup were provided with so-called semantic maps during the two lectures. A semantic map is a network of information parts that are mutually related. The subject-matter presented in the lectures was elucidated by these semantic maps. Two other subject groups, a low-knowledge and a high-knowledge subgroup, were shown a summary of the subject-matter during the two lectures, whereas the remaining low-knowledge and high-knowledge subgroups were presented with a list of key words while attending the lectures. Two days after studying the subject-matter a free recall task had to be carried out. The low-knowledge group that received a semantic map showed higher recall scores than the other two low-knowledge groups. Strikingly, the opposite was true for the high-knowledge groups. The subgroup that was presented with a list of key words recalled more of the subject-matter than the other two high-knowledge subgroups, whereas the lowest recall scores were obtained by the highknowledge subgroup that was provided with a semantic map.

According to Lambiotte and Dansereau, the information processing of low-knowledge subjects was facilitated as a result of the availability of a semantic map. This map provided them with a structure which could be used during encoding. High-knowledge subjects already possessed such a structure. Therefore, presenting a semantic map during information processing would not necessarily lead to a performance improvement for highknowledge subjects. On the contrary, this semantic map could possibly inter- 
fere with information processing. The way in which knowledge was structured in this map could differ from the structure the high-knowledge students had established themselves. Therefore, presenting a semantic map to subjects who were already familiar with the subject-matter could lead to confusions. Providing them with a list of key words, on the other hand, could have facilitation effects since these words may be expected to correspond with elements in the subjects' knowledge structure.

From Lambiotte and Dansereau's (1990) results, it could be concluded that low-knowledge subjects generally benefit more from advance organizers than high-knowledge subjects. Mayer (1976) already showed that the presentation of an advance organizer prior to studying a technical text resulted in larger profits for novices in a certain domain than for experts in that field.

Overall, it can be concluded that research by Ausubel $(1960,1963,1968)$ and others has shown that advance organizers can influence the learning process in a positive way. In other words, a relevant context is provided as a result of prior knowledge activation by means of an advance organizer, facilitating subsequent information processing. This appeared to be a strict encoding phenomenon.

However, Corkill, Glover, Bruning and Krug (1988) argued that the retrieval hypothesis cannot be rejected completely. They demonstrated that presenting an advance organizer after the study phase but preceding the recall phase also resulted in facilitation effects. These conclusions, however, only held when the interval between the study phase and the recall phase amounted to at least 24 hours. According to Corkill et al., these results provide support for the retrieval hypothesis, extended with the notions that (a) advance organizers can facilitate retrieval when recall is delayed, and (b) the recall phase is preceded by rereading the advance organizer. Rabinowitz and Craick (1986) share this point of view.

\section{THE CONTEXT EFFECTS STUDIES}

A variation on the use of an advance organizer is the presentation of a title or an illustration to provide a relevant context from which a text can be interpreted.

Bransford and Johnson (1972) presented subjects with a text that was difficult to understand if a title is lacking. An example of such a text is presented on the next page. This text was auditively presented to three subject groups. The first group was provided with an appropriate title for the text (i.e., "Washing clothes") prior to listening to the text. The second group received this elucidating title after the auditive presentation of the text. To the control group the title "Washing clothes" was not shown at all. 
The procedure is actually quite simple. First, you arrange items into different groups. Of course, one pile may be sufficient depending on how much there is to do. If you have to go somewhere else due to lack of facilities, that is the next step; otherwise, you are pretty well set. It is important not to overdo things. That is, it is better to do too few things at once than too many. In the short run this may not seem important but complications can easily arise. A mistake can be expensive as well. At first, the whole procedure will seem complicated. Soon, however, it will become just another facet of life. It is difficult to foresee any end to the necessity for this task in the immediate future, but then, one never can tell. After the procedure is completed one arranges the materials into different groups again. Then they can be put into their appropriate places. Eventually they will be used once more and the whole cycle will then have to be repeated. However, that is part of life.

The three subject groups had to judge the comprehensibility of the text on a 7point scale. Furthermore, each subject had to carry out a free recall test. The subjects who received the title "Washing clothes" prior to the presentation of the text showed higher scores on both tests than the two other subject groups. Thus, the subjects who were provided with the title immediately after the presentation of the text did not benefit from this contextual information at all; the recall performances of this group equaled the control group's scores. 1

In a second study of Bransford and Johnson (1972) an illustration was applied to create a relevant context. The text used in this study is printed on the next page. In this study again three subject groups participated: (a) The first subject group was, prior to listening to the text, provided with an illustration which created an appropriate context for the text; (b) the second group first listened to the text and was subsequently shown this picture that illuminated the text; and (c) the third, control, group received no congruent contextual information at all.

1 A criticism with respect to this study is put forward by Bransford (1979) himself. In the "Washing clothes" study, the so"called given-new contract (Haviland \& Clark, 1974) may" have been violated. In daily life, a kind of agreement exists between speakers and listeners. When the speaker, for instance, uses a definite article ("the") to refer to something introduced previously, this object indeed has to be mentioned in the preceding conversation. This contract was violated since in the text presented is referred to something that ought to be known ("Washing clothes") but that was not mentioned explicitly. Alba, Alexander", Hasher" and Caniglia (1981) take the same stand in this issue. Bransford (1979), however, considers. this violation as not detrimental to the value of the study since in daily life many conversations are incomplete and can only be correctly interpreted when the listener actively uses his or her prior knowledge to fill the gaps. From this perspective, he considers the study as ecological valid. 
If the balloons popped, the sound would not be able to carry since everything would be too far away from the correct floor. A closed window would also prevent the sound from carrying since most buildings tend to be well insulated. Since the whole operation depends on a steady flow of electricity, a break in the middle of the wire would also cause problems. Of course, the fellow could shout, but the human voice is not loud enough to carry that far. An additional problem is that a string could break on the instrument. Then there could be no accompaniment to the message. It is clear that the best situation involves less distance. Then there would be fewer potential problems. With face to face contact, the least number of things could go wrong.

The illustration providing an appropriate context for the text shows a modern-day scene from Shakespeare's Romeo and Juliet; the latter is leaning out of a window on the top floor of an apartment. Her lover on the ground floor serenades her on his guitar. To bring Juliet on cloud seven, she would have to be able to perceive Romeo's serenade; however, the distance between the lovers is too large. To resolve this problem, a loud-speaker, connected to Romeo's microphone, is lifted into the air by means of a number of balloons.

Subjects had to score the comprehensibility of the text on a 7-point scale, which was immediately followed by a free recall test. With respect to both tests, the subjects whom the illustration was presented before listening to the text outperformed the other two groups. When presented after hearing the text, the picture did not have any facilitating effect; the scores of this group on the comprehensibility test as well as on the recall test did not exceed the scores obtained by the control group.

Thus, prior knowledge activation by means of a clarifying title or an illuminating picture presented prior to the study task facilitated information processing. No facilitation effects were found when this kind of contextual inagreement with Mayer and Bromage's (1980) conclusions that the facilitative effects of providing a relevant context seems to be restrained to the encoding phase. Bransford (1979), therefore, concluded that prior knowledge should not just merely be available, but that this knowledge has to be actively applied during subsequent information processing. This view was subscribed by
Perfetto, Bransford and Franks (1983).

\section{THE ROLE OF DOMAIN KNOWLEDGE}

In the research reported in the previous paragraphs, it was shown that manipulating prior knowledge has a large impact on subsequent information 
processing. However, Anderson, Reynolds, Schallert and Goetz (1977) demonstrated that information processing is also influenced by existing knowledge structures without active interventions by the researchers. In this respect, two categories of experiments can largely be discerned: (a) Studies in which subjects possessed knowledge in different domains, and (b) studies in which subjects had limited versus extended knowledge in a particular domain (Chiesi, Spilich, \& Voss, 1979; Spilich et al., 1979).

An example of the first category of studies stems from Anderson et al. (1977). In their experiment, two texts were applied which could be interpreted in two different ways. One of these multi-interpretable texts is:

Rocky slowly got up from the mat, planning his escape. He hesitated a moment and thought. Things were not going well. What bothered him most was being held, especially since the charge against him had been weak. He considered his present situation. The lock that held him was strong but he thought he could break it. He knew, however, that his timing would have to be perfect. Rocky was aware that it was because of his early roughness that he had been penalized so severely - much too severely from his point of view. The situation was becoming frustrating; the pressure had been grinding on him for too long. He was being ridden unmercifully. Rocky was getting angry now. He felt he was ready to make his move. He knew that his success or failure would depend on what he did in the next few seconds.

This story could be perceived to be about a convict in his cell planning his escape, the text's primary reading according to Anderson et al. The text could also reflect the story of a wrestler, trying to break the hold of his opponent, which was labeled by Anderson et al. as the text's secondary meaning."

The second passage applied in the Anderson et al. (1977) study is presented on the next page. The primary meaning of this passage would reflect a social evening of cards; the secondary, less obvious reading would be a description of a woodwind ensemble's rehearsal.

The subjects participating in this study came from two different institutes. The first group of subjects were physical education students and the second group consisted of music school students. After reading the text, each subject carried out both a multiple-choice test and a free recall test. Scores on

1 It should be borne in mind that in 1977 the name "Rocky" was not yet associated with boxers, fighting, et cetera. Now, in 1993, after a number of "Rocky" films, this name would probably result in favoring the text's secondary meaning, irrespective whether or not the reader possessed specific domain knowledge. 
both tests revealed a strong relationship between the subjects" background and their interpretation of the text. Because of their domain knowledge, the physical education students had interpreted the Rocky text as being the reflection of a wrestling game, which was in agreement with the text's secondary meaning. "The music students, however, read that text in a different way. Both their answers on the multiple-choice test and their free recall protocols showed that these subjects implicitly had interpreted the text as a description of a prisoner in his cell, which the researchers labeled as the text's primary meaning. The opposite was found for the second story. Based on their background knowledge, the music students had chosen the text's secondary meaning, describing the rehearsal of a woodwind ensemble. On the other hand, this text passage was interpreted by the physical education students as a reflection of an evening of cards, which, according to the researchers, corresponded with the primary meaning of the text passage.

Every Saturday night four good friends get together. When Jerry, Mike, and Pat arrived, Karen was sitting in her living room writing some notes. She quickly gathered the cards and stood up to greet her friends at the door. They followed her into the living room but as usual they could not agree on exactly what to play. Jerry eventually took a stand and set things up. Finally, they began to play. Karen's recorder filled the room with soft and pleasant music. Early in the evening, Mike noticed Pat's hand and the many diamonds. As the night progressed the tempo of the play increased. Finally, a lull in the activities occurred. Taking advantage of this, Jerry pondered the arrangement in front of him. Mike interrupted Jerry's reverie and said, "Let's hear the score." They listened carefully and commented on their performance. When the comments were all heard, exhausted but happy, Karen's friends went home.

The studies outlined show that the nature of domain knowledge influences text interpretation and recall. Text recall is not only determined by the nature of domain knowledge (Chiesi et al., 1979), but it is also influenced by the amount of domain knowledge. Spilich et al. (1979), for example, auditively presented both subjects with little knowledge of baseball and subjects whose knowledge in this domain was extended with a half-inning account of a fictional baseball game. Afterwards, they had to write down as much as they could recall from the entire account; subsequently, 40 completion questions related to specific account information had to be answered. High-knowledge subjects not only recalled more of the game, but they also tended to integrate sequences of goal-related actions. Furthermore, they recalled more information in the appropriate order than low-knowledge subjects, who often omitted 
significant information. The questionnaire also showed better performances of the high-knowledge subjects, attributable to higher scores for questions referring to major game actions.

In summary, existing prior knowlledge appeared to have facilitative effects on subsequent information processing and recall.

\section{PERSPECTIVE ASSIGNMENT}

In 1977, Pichert and Anderson showed that facilitation effects could be found if prior knowledge was activated by perspective assignment; in other words, by telling subjects from which point of view a text should be read. The text applied in their study is the following:

The two boys ran until they came to the driveway. "See, I told you today was good for skipping school," said Mark. "Mom is never home on Thursday," he added. Tall hedges hid the house from the road so the pair strolled across the finely landscaped yard. "I never knew your place was so big," said Pete. "Yeah, but it"s nicer now than it used to be since Dad had the new stone siding put on and added the fireplace." There were front and back doors and a side door which led to the garage which was empty except for three parked 10-speed bikes. They went in the side door, Mark explaining that it was always open in case his younger sisters got home earlier than their mother. Pete wanted to see the house so Mark started with the living room. It, like the rest of downstairs, was newly painted. Mark turned on the stereo, the noise of which worried Pete. "Don't worry, the nearest house is a quarter of a mile away," Mark shouted. Pete felt more comfortable observing that no houses could be seen in any direction beyond the huge yard. The dining room, with all the china, silver and cut glass, was no place to play so the boys moved into the kitchen where they made sandwiches. Mark said they wouldn't go to the basement because it had been damp and musty ever since the new plumbing had been installed. "This is where my Dad keeps his famous paintings and his coin collection," Mark said as they peered into the den. Mark bragged that he could get spending money whenever he needed it since he d discovered that his Dad kept a lot in the desk drawer. There were three upstairs bedrooms. Mark showed Pete his mother's closet which was filled with furs and the locked box which held her jewels. His sister's' room was uninteresting except for the color TV which Mark carried to his room. Mark bragged that the bathroom in the hall was his since one had been added to his sisters' room for their use. The big highlight in his room, though, was a leak in the ceiling where the old roof had finally rotted. 
This text contains information that was relevant either from the perspective of a potential homebuyer or from a burglar's perspective. Subjects in this study were randomly assigned to one of three groups: (a) The first group was instructed to read the text from the perspective of a potential homebuyer; (b) the second group was told to read the text from the burglar perspective; and (c) the third group, the control group, did not read the story from a directed perspective. The study phase was followed by a free recall test, in which the subjects explicitly were requested to report everything they could recall from the text. Information that was relevant to the assigned perspecive was recalled better than information irrelevant to the directed perspective.

According to Pichert and Anderson (1977), these results support the socalled schema theory (Anderson, 1977; Rumelhart \& Ortony, 1977). Assigning a perspective from which a text should be read would result in the activation of a an appropriate schema, which guides subsequent information processing. A schema can be defined as an abstract knowledge structure containing information about frequently experienced situations, events, et cetera (Anderson, 1990). This knowledge structure contains slots (placeholders or variables) in which information can be incorporated. A schema characterizes the typical relationships among its elements. A schema is instantiated by filling its sllots with values. which can vary within a restricted range. Some of these slots can be filled with inferences (default assignments). ${ }^{1}$ From the assumptions of the schema theory, predictions can be formulated about the way in which information is processed and subsequently recalled. According to Anderson (1982), during information processing a distinction is made between information relevant to the schema previously activated and information that is irrelevant in this respect.

The question now arises whether or not this process of selecting slotfilling information requires extra processing time. According to the so-called selective-attention hypothesis, which is based on the assumptions of the schema theory, information corresponding with slots of the activated schema is recalled better as a result of the additional processing time spent on this information (Alba \& Hasher, 1983). On the other hand, the so-called slot-filling hypothesis provides an alternative explanation for the selection process emerging from schema activation. This hypothesis also predicts that information corresponding with the activated schema is recalled best; however, this would not necessarily involve higher reading times. Recall of perspective relevant information would simply be better because the schema contains slots for this information. No additional study time would be required to in-

1 Other terms reterring to knowledge representations describing common, stereotype situations are "rame" (Minsky, 1975), "script" (Schank \& Abelson, 1977) and "scenario" (Sanford \& Garrod, 1981). 
tegrate this information into the schema; on the contrary, it would be considered likely that processing slot-filling information would take less time.

Pichert and Anderson (1977) were not able to test these explanations for the selection process since in their research study times for each information type had not been registered. Data provided by Goetz, Schallert, Reynolds and Radin (1983) fulfilled this need. Pichert and Anderson's (1977) text was applied in their study. Each sentence of this text was presented on a computer screen. After studying each sentence, subjects pressed a key on the console. Automatically this sentence was erased and immediately, the next one appeared. Reading times for each sentence were registered. Prior knowledge was activated by assigning (burglar or homebuyer) perspectives. Again, information relevant to the assigned perspective was recalled better than information irrelevant in this respect. But what was more compelling, sentences containing perspective relevant information required more time than sentences containing perspective irrelevant information. Schema activation seems to result in directing the reader during text processing to information that is relevant regarding the assigned perspective, which was therefore studied longer. Consequently, this information was recalled best. These findings were replicated by Vonk (1986), applying an extended version of Pichert and Anderson's (1977) homebuyer-burglar text. Furthermore, Vonk found similar recall patterns for two new texts, which could also be read from two different perspectives: Slot-filling information was processed longer, resulting in higher recall scores for that information. 1

No confirming evidence for the selective-attention hypothesis was found by Grabe (1979) and Kardash, Royer and Greene (1988). In both studies, in which Pichert and Anderson's (1977) text was applied, prior knowledge was activated by assigning perspectives. Again, perspective relevant information was recalled better than irrelevant information, but perspective relevant information did not require more processing time. Therefore, the results of Grabe (1979) and Kardash et al. (1988) support the slot-filling hypothesis.

Thus, the controversy whether or not the selection process induced by schema activation requires additional processing time or not is still unresolved since support is provided for both the selective-attention hypothesis and the slot-filling hypothesis; strikingly, this evidence is acquired by applying the same study text (Pichert \& Anderson, 1977; Anderson \& Pichert, 1978).

\footnotetext{
1 Studies by Anderson and Pearson (1984), Reymolds, Standiford and Anderson (1979) and Reynolds and Anderson (1982) also support the selective-attention hypothesis. During text processing, subjects" attention was directed to information providing answers to a number of questions, which the subjects had received prior to text processing. Further research in which subjects were presented with questions prior or during the study phase, resulting in selecting information congruous with these questions can be derived from Voss, Tyler and Bisanz (1982).
} 
In summary, prior knowledge activation by means of assigning perspectives from which a text should be read is assumed to result in schema activation, instilling the selection of slot-filling information during subsequent information processing. Consequently, information corresponding with the knowledge previously activated would be recalled best.

\section{PRIOR KNOWLEDGE MOBILIZATION}

The outcomes of a number of recent studies are difficult to interpret within the schema-theoretical framework. These studies were conducted within the prior knowledge mobilization paradigm, which was independently developed by Peeck (1982) and Schmidt (1982a). Prior to reading a text, subjects are encouraged to bring to mind all knowledge they have in a certain domain. Subsequently, relevant new information is provided.

Peeck, Van den Bosch and Kreupeling (1982), for instance, required an experimental group of elementary school children to activate prior knowledge by generating in writing everything they knew about the appearance and habits of the fox. A control group conducted a similar activity with respect to farm animals and farm activities. Subsequently, a short text (125 words) about the appearance and habits of a fictional animal, the American heath fox, was read by both groups of subjects. Three information categories could be distinguished: (a) Information that was congruent with the pupils' knowledge of the fox (congruous information), (b) information that was in contradiction with their knowledge (incongruous information), and (c) neutral information not conflicting with the prior knowledge. The experimental group outperformed the control group in recall of both incongruous and neutral information; the recall scores of the experimental group did not surpass those of the control group with respect to congruous information. Thus, prior knowledge activation through mobilization resulted in higher recall scores for information for which no activation previously took place.

Schmidt (1982a) applied a somewhat different prior knowledge activation strategy. An experimental group, subdivided into three subgroups, discussed the following problem: "A red blood cell (a red blood corpuscle) is put into pure water under a microscope. The blood cell swells rapidly and eventually bursts. Another blood cell is placed in a solution of salt in water. It shrinks. How can these phenomena be explained?" Each subgroup had to come up with explanations for the problem described. The control group, also subdivided into three groups, analyzed in the same way a problem describing how airplanes seem to withdraw from the law of gravitation. After prior knowledge activation by means of problem-analysis, each subject studied a text about osmosis and diffusion ( 2200 words). These processes underlie the blood cell phenomena which the experimental group had to ex- 
plain. The study phase was pursued by a recognition test and a transfer test. The recognition test consisted of correct and incorrect items, concerning paraphrases and transformations of study text elements. The transfer test consisted of problems which could be solved by information in the text; this test also contained inferences (i.e., statements that are not explicitly mentioned in the text, but that can be inferred from it). The experimental group showed higher scores on both the recognition test and the transfer test. For a subsequent analysis, the items were split into items that were directly relevant to the prior knowledge previously activated during problem-analysis, and items that were not directly linked to the prior knowledge but that could be associated with this knowledge. This analysis revealed that the differences between the two groups of subjects could be attributed to higher scores of the experimental group for items that were not directly related to the prior knowledge activated in the problem previously analyzed.

Similar results were obtained by Peeck (1982). In Peeck's experiment prior knowledge was activated by means of generating names of either American presidents (presidents group) or states of the Union (states group). The control group had to mobilize as many names of animals as possible. Subsequently, each subject was presented with an item list consisting of 16 president names and 16 states of the Union. The subjects were instructed to try to recall as many items of the list as possible. A free recall test was administered either after 15 minutes or after 24 hours. In both conditions, the presidents group appeared to have recalled more president names than the control group. The states group outperformed the control group in recall of state items. With respect to the "unfamiliar" item category (i.e., the item category that had not been mobilized previously), no recall differences were found between the experimental groups on the one hand and the control group on the other hand. The higher recall performances of the two experimental groups appeared to result from better recall of items of the category previously activated but that were not generated during the mobilization phase.

How can these recall patterns be accounted for? Based on their findings, Peeck et al. (1982) concluded that prior knowledge mobilization had a broad "stage-setting" function (Bransford \& Franks, 1976), extending the effect of prior knowledge activation to non-mobilized information. Peeck et al. find their results consistent with the assimilation encoding theory (Mayer, 1979; Mayer \& Bromage, 1980), predicting higher recall scores if a rellevant context is available in memory which could be actively used during information processing in which the new information can be integrated. The prior knowledge mobilized in the Peeck et al. study would serve as such a context.

However, this explanation does not account for the fact that the experimental groups did not outperform the control groups in recall of information corresponding with the knowledge previously activated. The overall higher 
recall scores were solely attributable to better recall of information that had not been previously activated.

An alternative explanation stems from Peeck (1982), who assumed that subjects were attracted by the novelty of the information that had not been previously activated, resulting in higher recall scores for that information. Peeck subscribed Kintsch's (1980) statement: "It is change, incongruity, surprise that leads to new learning ... Misfits between the apperceptive mass and new information ... provide the right conditions for learning, which is now conceived of as a correction or addition to existing knowledge structures" (pp. $92-93$ ) "1

However, again the recall patterns obtained cannot be fully accounted for by the novelty of incongruous information since it can be presumed that this information is considered as deviant by the control group as well. Peeck (1982) already suggests that "the mobilization group appears to have been especially conducive to the detection of misfits and knowledge gaps by making the learner more aware of his or her initial existing knowledge structure" (p. 611). Conflicting information would thus only be processed to a deeper level when the subject is aware of the information's incongruity with his or her existing knowledge. As a consequence of activating prior knowledge the experimental group would have been aware of the contradiction between the incongruous information and the prior knowledge. This would apply to the control group in a lesser extent since this group did not mobilize knowledge related to the study text.

This assumption appears to fill gaps in Peeck's (1982) explanation for the recall patterns found. Yet, also the novelty hypothesis cannot explain all findings. In the study of Peeck et al. (1982), the experimental group not only showed higher recall scores for incongruous information, but also with respect to information that was neither congruous nor incongruous with the knowledge previously mobilized, but neutral to this knowledge. It does not seem likely that the experimental group would have been more aware of the neutrality of that information than the control group as a result of prior knowledge activation.

An explanation for the experimental groups' higher recall performances, which could largely be attributed to better recall of information for which no activation previously took place seeks Schmidt (1983a, 1984) in the way in which study time was allocated to the various parts of the study text. In order to explain the recall phenomenon, Schmidt proposed a cognitive set-point hypothesis. This hypothesis assumes that during information processing a

\footnotetext{
1 Peeck"s (1982) novelty hypothesis is supported by a number of studies, showing that information atypical to a certain script is recalled better than information that could be considered as script typical (Bower, Black, \& Turner, 1979; Graesser, Gordon, \& Sawyer, 1979; Pezdek, Whetstone, Reynolds, Askari, \& Dougherty, 1989).
} 
cognitive set-point is applied, indicating when sufficient study time has been spent on processing certain information in order to recall it at a later stage. The cognitive set-point is reached when a certain amount of time has been spent on processing the information. So, study time is a crucial factor in the cognitive set-point hypothesis. A description of an imaginary experiment may elucidate this hypothesis and, therefore, Schmidt's explanation for the recall patterns observed in the studies of Peeck (1982), Peeck et al. (1982) and Schmidt (1982a, 1982b).

Let's assume a study in which an experimental group mobilizes relevant prior knowledge and a control group who does not. In this study a text is presented of which a certain information part corresponds with the prior knowledge previously mobilized by the experimental group. Both groups have to study this text within a fixed amount of time. According to the cognitive setpoint hypothesis, the cognitive set-point dictates when sufficient processing time is spent on certain information in order to recall it. During mobilization the experimental group mobilized certain knowledge and thus, already started processing the information corresponding with this prior knowledge. If this information is subsequently encountered in the study text, relatively less processing time would be required to reach the cognitive set-point for this information type, compared to the control group that did not mobilize relevant prior knowledge. Thus, during the study phase relatively less processing time would be required to reach the cognitive set-point as a result of prior knowledge mobilization.

If the total amount of study time available for text processing is fixed which could freely be distributed over the various information parts of the text, like in this imaginary study, the experimental group would remain relatively more study time than the control group to process new information for which no activation took place as a result of activating relevant prior knowledge. Consequently, the experimental group would be able to reach the setpoint for a large part of this non-mobilized information too. Since the control group would have to spend more time on the information part in the text corresponding with the prior knowledge mobilized by the experimental group in order to reach the cognitive set-point for that information, this group would not remain sufficient processing time to reach the set-point for information that had not been mobilized by the experimental group. This information process would induce the recall patterns as observed in the studies of Schmidt (1982a, 1982b), Peeck (1982) and Peeck et al. (1982): The experimental group outperformed the control group in recall of non-activated information, whereas the two groups did not differ in recall of information corresponding with the prior knowledge mobilized by the experimental group.

In summary, the cognitive set-point hypothesis predicts two effects emerging from mobilizing relevant prior knowledge. First, since relatively 
more processing time could be spent on non-mobilized information the experimental group would be able to reach the cognitive set-point for this information; consequently, the experimental group is assumed to outperform the control group in recall of information for which no activation took place, resulting in overall higher recall scores. Second, given that total study time is fixed, the experimental group is expected to show a pattern of study time allocation that is divergent from that of the control group. Processing time for information corresponding with the prior knowledge activated would be shorter since the experimental group would be able reach the cognitive setpoint for this information sooner than the control group. Consequently, the experimental group could process new relevant information that had not been mobilized longer than the control group.

Based on these assumptions of the cognitive set-point hypothesis, different patterns of study time allocation and recall could be predicted if available total study time would not be limited in any way. Under this processing condition the experimental group would require less study time for text processing than the control group, which particularly would hold for information corresponding with the knowledge activated. The control group would also be able to reach the set-point for this information by studying this information longer. Consequently, the control group is assumed to show recall scores equal to those of the experimental group if total study time is free.

Data provided by Johnson and Kieras (1983) seem to support the latter predictions when total study time was free. In Johnson and Kieras' study, subjects were confronted twice with short texts. The sentences of each text were presented one by one on a computer screen. During the first presentation of a text, subjects had to indicate if the particular sentence contained information previously known; the second time, they had to study each sentence of the texts in order to recall it. Study times tended to be longer for sentences containing information that subjects had rated as previously unknown. No differences in recall were found between previously known information and previously unknown information. ${ }^{1}$ These results are in line with the predictions of the cognitive set-point hypothesis. Yet, it should be mentioned that the effects in Johnson and Kieras' study were rather small.

If information would be presented with a fixed rate, processing times, of course, would not differ. Moreover, no differences in recall would arise. The experimental group would no longer be able to benefit from its processing advantage stemming from prior knowledge mobilization since time spent on

\footnotetext{
1 Johnson and Kieras (1983) were aware that presenting subjects twice with the same study material could weaken the effects of prior knowledge; however, only low correlations were obtained between sentence rating and rating time, whereas sentence rating highly correlated with study time. Therefore, Johnson and Kieras concluded that sentences were not intentionally studied while rating them.
} 
information previously mobilized could not be reduced nor could more time be spend on non-mobilized information.

Thus, the cognitive set-point hypothesis would provide an explanation for the findings of Peeck (1982), Peeck et al. (1982) and Schmidt (1982a, $1982 \mathrm{~b})$. Although the recall patterns obtained in these studies support the cognitive set-point hypothesis under the condition that total study time is fixed which can be freely distributed over the various information parts of a text, a more conclusive test is needed since these studies do not provide information with regard to the allocation of study time.

\section{THE SELECTIVE-ATTENTION VERSUS THE COGNITIVE SET-POINT HYYPOTHESIS}

Strikingly, although both the selective-attention hypothesis and the cognitive set-point hypothesis predict facilitation effects of prior knowledge activation on subsequent information processing and recall, they differ in the way these facilitation effects emerge. According to the selective-attention hypothesis, prior knowledge activation results in better recall of information corresponding with the knowledge previously activated (Anderson, Pichert, \& Shirey, 1983; Berman, Read, \& Kenny, 1983; Cohen, 1981), whereas the cognitive set-point hypothesis claims that prior knowledge activation leads to higher recall performances for information for which no activation previously took place (Schmidt, 1983a, 1984).

What may be the reason for these discrepancies? First, the studies on which these hypotheses are based cannot be compared easily. For instance, the contradictory results obtained in these experiments could result from the different ways in which prior knowledge was activated. In studies providing support for the selective-attention hypothesis, prior knowledge was activated by means of assigning a perspective from which a text should be read. By means of this strategy, prior knowledge activation remains largely implicit. In research supporting the cognitive set-point hypothesis, subjects were $e x$ plicitly requested to mobilize all knowledge they possessed in a certain domain. These different techniques may have induced the different recall patterns predicted by the two hypotheses. Second, the nature of the knowledge previously activated may have been different in the two sets of experiments. Activating a burglar schema, as in Pichert and Anderson's (1977) study, seem to be rather deviant from the knowledge that was activated in, for instance, Schmidt's (1982a) research. The knowledge activated in Pichert and Anderson's (1977) study (a homebuyer or a burglar schema) would be familiar to almost everyone and largely consisted of elements shared by almost anybody. The study material suited this schematic knowledge. Subjects would not have to struggle to understand the texts applied. As a result of perspective 
assignment the appropriate schema was already present, which merely would have to be up-dated by filling its slots with matching information from the text. In Schmidt's (1982a) research, however, both the knowledge previously activated and the study material appear to be of a completely different nature. In this experiment, subjects knew relatively little of osmosis and diffusion. They did not possess a well-structured knowledge representation, such as a schema, which fitted the text presented. Therefore, information could not be simply integrated. On the contrary, in order to extend their limited knowledge in this domain they would have to actively relate this prior knowledge to the new information. In other words, subjects could not just up-date existing knowledge by filling slots in an existing schema, but they actually learned new information and extended in this way their knowledge in the particular domain. If prior knowledge is limited, and, thus, a readymade cognitive structure would be lacking, information processing can be viewed as laborious and non-automatic.

In summary, two reasons may exist accounting for the divergent findings providing support for either the cognitive set-point hypothesis or the selective-attention hypothesis: (a) Differences in the operationalization of prior knowledge activation; and (b) differences in the nature of the prior knowledge activated, which would subsequently trigger different ways of information processing (Anderson, 1983, 1987; Kintsch, 1988; Rumelhart \& Norman, 1978; Schmidt \& Boshuizen, 1992; Shuell, 1990). A short intermezzo depicting the way in which knowledge is acquired may enlighten the latter assumption.

\section{THE ACQUISITION OF KNOWLEDGE}

In the late 1970s, the dominant perspective was that comprehension of the world was identical with schema instantiation (Norman, Rumelhart, \& the LNR Research Group, 1975; Rumelhart \& Norman, 1978). A schema is activated by the situation encountered. During information processing, the slots of this schema are filled with vallues, stemming from the present situation. Thanks to the already existing schema the particular situation can be dealt with. However, if a schema appears not to be apt to fully cover the situation that activated it, the original schema has to be adjusted in order to account for this new situation. Rumelhart and Norman (1978) apply the term "accretion" to refer to this process in which the original schema is extended, although its initial structure is largely maintained. Rumelhart and Norman describe two other circumstances under which schemata can change, but in these cases the alterations go beyond merely extending the original schema. The expression "tuning" refers to changes of the original schema as a result of growing experience. The way in which these changes evolve largely depends 
on the way in which the experience is manifested. If the process of experience evolves vertically (i.e., a large number of experiences within the same range), specialization occurs. If this process is horizontally developed (i.e., the experiences also pertain to related aspects), generalization takes place. As a result of generalization the spectrum of values that schema slots can take becomes wider, whereas in the case of specialization this spectrum becomes more restricted. A more drastic modification of the initial schema is required when the activated schema cannot account for the situation in question at all. Rumelhart and Norman refer to this process with the term "restructuring".

In general, information comprehension is in this model viewed as a topdown, expectation-driven process. The existing knowledge determines the interpretation of information. The appropriate meaning of information filters through; the inappropriate meanings are suppressed by the existing knowledge. This way of information processing appears to be quite efficient. However, this model cannot account for every form of learning that actually takes place. The starting point of Rumelhart and Norman's (1978) model is the presence of a schema through which information can be understood. But what about non-prototypic situations for which no schemata exist? According to their model, learning something that one did not already know would be impossible, and yet, it (fortunately) happens all the time. In addition, schemata are far too rigid knowledge structures to account for the complexity of actual learning taking place (Schmidt \& Boshuizen, 1992).

This critique was also acknowledged by Kintsch (1988). In introducing his construction-integration model Kintsch takes the opposite stand since this model is based on bottom-up information processing. Existing knowledge of the world is represented by an associative network of nodes and interrelations, the so-called knowledge base. Such a network is far more flexible than a schema since its concepts (or propositions) are loosely interrelated. While studying a text, words or phrases in this text will activate corresponding nodes and activation will spread to related nodes. In this way, a pool of knowledge is activated that may or may not be relevant to the task of understanding the particular text. The irrelevant knowledge activated in this construction phase is constrained by more information from the text and, hence, activation of further concepts and interrelations. Thus, in this integration phase a coherent text representation is formed by integrating knowledge from earlier cycles of activation with that of later cycles, resulting in a so-called text base. In order to create such a coherent text base, the reader may have to produce bridging inferences and build macropropositions. Thus, the meaning of a text is an integration of existing prior knowledge and information from that text. In this way, the initial knowledge base is extended.

Theoretically, this expansion process of the original knowledge base, learning, may proceed for as long as new information within a certain do- 
main is encountered. Naturally, information processing would not be very efficient if such an immense knowledge network is activated. According to Anderson (1983), with growing experience, and thus knowledge, the existing knowledge representation can be transformed into a more efficient structure.

Anderson's $(1983,1987)$ ACT $^{*}$ theory is based on the assumption that human cognition is fundamentally a production system. In this system, knowledge is represented in an associative network consisting of declarative propositions, containing world knowledge, facts derived from study texts, et cetera. When this knowledge is applied for the first time, for instance, to solve a problem, mastering this task is difficult since an extensive search is required. With growing experience, this procedure will become more efficient; a proceduralization process emerges. While searching for the right solution for the problem at hand, the conditions under which it appeared to be appropriate are encoded in memory. Eventually, this will lead to the development of so-called production rules (or, condition-action rules), representing the knowledge required for adequate solving that particular type of problems. A second efficacy process can occur after another series of successfully applying this knowledge. For instance, if two production rules appeared to apply one after another, a single rule can be constructed in which the effects of the two original rules are combined (composition). Through these processes, building production rules (proceduralization) and composition, knowledge will become compiled. Knowledge compilation refers to a more efficacious way of information processing and problem solving (Boshuizen, 1989). Declarative knowledge has no longer to be retrieved in working memory to master a particular task; the essential products, resulting from many previous encounters is accessed, providing the correct solution (Holyoak, 1990).

Schmidt and Boshuizen (1992) do not make the assumption that knowledge can only compile when in a production system mode. They assume that associative nets consisting of declarative knowledge become encapsulated under the influence of experience. Knowledge encapsulation is a process analogous to Anderson's $(1983,1987)$ compilation. It is defined as "the subsumption or packaging of lower-level, detailed propositions, concepts and interrelations in an associative net, under a smaller number of higher-level propositions with the same explanatory power" (p. 3). As experience increases, the knowledge network elaborates much in the way described by Kintsch (1988). After many applications of this elaborate knowledge, encapsulation takes place; i.e., a (part of the) network can be captured in one higherlevel proposition, in which all the subordinate propositions are included. Information processing and problem solving would be more efficient if one has the disposal of knowledge structured in such a compact way.

Like Schmidt and Boshuizen (1992), Shuell (1990) describes learning as a process that in its earlier stages is predominantly characterized by bottom-up 
processing, whereas latter stages of learning may be more top-down in nature. According to Shuell (1990), the learner has to go through three phases of meaningfull learning to reach the ultimate phase, which is comparable with Anderson's (1983, 1987) knowledge compilation and Schmidt and Boshuizen's (1992) knowledge encapsulation. In the first phase of meaningful learning, the learner is not yet familiar with the knowledge domain. During information processing he acts like a novice since specific domain knowledge is still lacking. Isolated facts are memorized; existing knowledge structures of other domains are applied to interpret the new information, to identify similarities and contrasts, to find analogies, et cetera. The newly acquired information is bound to the specific context in which it was encountered. Gradually, an overview of the new domain's content arises. In this intermediate phase, relationships are built between the isolated information pieces, acquired in the initial phase of learning. Eventually, these relationships become more abstract. These higher-order structures can be used during subsequent information processing. The knowledge becomes more abstract since it is applied to all kinds of situations. Thanks to this generalization, the knowledge becomes less dependent on the context in wich it was obtained. However, automatic information processing is still impossible. 1 During the final phase the knowledge structures become more integrated and work more automatically; in other words, the state of schema has been reached. Mastering tasks (e.g., answering questions, solving problems) will be effortless and automatic. In this phase, learning consists of adding new facts to the existing cognitive structures or acquiring higher levels of interrelationships between elements of this cognitive structure.

Shuell (1990) provides an example of the latter form of learning, occurring in the terminal phase of meaningful learning. Increasingly higher levels of interrelationships evolve when a schema no longer consists of facts filling the schema slots, but when these slots are filled with new schemata. Such a higher-level schema incorporates general, abstract information, applying to a large number of situations, whereas the schemata filling the slots of this higher-level schema apply to specific situations. Thus, in this terminal phase of meaningful learning, increasing experience results in the creation of highlevel cognitive structures, dominating subordinate structures; the relationships between elements of these structures are more or less fixed.

Evidence for this efficient way of information processing can be derived from experts mastering tasks. Hardly any effort is required, and their reac-

1 On the contrary, since the knowledge is applied to a huge variety of situations, it can be used in inappropriate situations, causing a number of processing problems (misconceptions). Naturally, knowledge abstraction can also be established by reducing the number of adequate application situations. However, this way of imformation processing would still require cognitive effort. 
tions come promptly. Their domain-specific knowledge has evolved into an efficient cognitive structure through compilation (Anderson, 1983) or encapsulation (Schmidt \& Boshuizen, 1992). The problem activates this network as one unit, from which the answer can be derived. Experts often provide the correct solution without being able to indicate precisely how it was achieved. The exact steps of this process cannot be explained; they just know. 1

Experts in various domains appeared to apply these encapsulated knowledge structures while solving problems or answering questions. For instance, experts in geometry used fixed cognitive structures (diagram configurations) to solve geometrical problems (Koedinger \& Anderson, 1990). Diagnostic abilities of prospective doctors were exceeded by the diagnostic performances of their more experienced colleagues since the latter possessed certain knowledge structures (illness scripts), obtained through innumerable patient visits (Hobus, Hofstra, Boshuizen, \& Schmidt, 1988). Robertson (1990) demonstrated that experts in solving physics problems applied fixed cognitive structures, resulting from years of experience.

\section{DIVERGENT INFORMATION PROCESSES}

The intermezzo depicted may show that contradictory models exist about the process of knowledge acquisition. The model of Rumelhart and Norman (1978) is strictly top-down and expectation-based, whereas the constructionintegration model of Kintsch (1988), stemming from his criticism on schemadriven information processing, is solely bottom-up. These conceptions about the way of information processing seem to be diametrically opposed. However, it could be assumed that both visions reflect two different phases in the process of learning as suggested by Schmidt and Boshuizen (1992) and Shuell (1990). Bottom-up information processing would occur if one's knowledge in a particular domain is (still) rather limited; information processing could then be compared to the way in which a novice in a certain domain solves problems: Laborious and requiring much cognitive effort. Top-down information processing, on the other hand, would take place if one's knowledge in a particular domain is extended; information processing would then resemble an expert in a particular domain solving problems: Smooth and almost automatic.

In the studies providing support for the selective-attention hypothesis (Anderson \& Pichert, 1978; Goetz et al., 1983; Lintsen, 1984; Pichert \& Anderson, 1977; Vonk, 1986), subjects acted like experts to some extent. As a result

1 This does not imply that lower-level knowledge has disappeared completely. On the contrary, if this knowledge is required (e.g., to solve an atypical problem), it can still be tracked
down. 
of the knowledge previously activated, a schema was activated, which provided a ready-made cognitive structure that could be used during subsequent information processing. ${ }^{1}$ Knowledge that is assumed to be common to almost anybody through films, newspapers, games, et cetera is represented in such a cognitive structure, e.g., the homebuyer or burglar schemata. A burglar schema would almost automatically induce aspects like "ways to enter the premises", "the loot", "routes to escape", et cetera. Information processing is presumed to be rather simple if such a schema is activated since the slots of the schema already exist; these slots would only have to be filled with appropriate values during information processing. Thus, the activated schema would merely have to be up-dated by filling the pre-existing slots with values selected during information processing. Activating a burglar schema would require a specification of the slot "loot" with values in the text like "furs" or "coin collection". Selection of this slot-filling information and incorporation of it into the schema previously activated would result in better recall of this information, which reflects the predictions of the selective-attention hypothesis.

On the other hand, in research providing support for the cognitive setpoint hypothesis (Peeck, 1982; Peeck et al., 1982; Schmidt, 1982a, 1983a) the way of information processing that would result from the prior knowledge previously activated would be typical for a novice in a certain domain. Prior knowledge was usually activated by means of mobilization. This task mostly referred to topics of which the subjects' knowledge was limited. They had not encountered this particular topic frequently; therefore, no elaborate or even encapsulated cognitive structures had yet emerged (Schmidt \& Boshuizen, 1992). In Schmidt's (1982a) study, for instance, the subjects' knowledge of osmosis and diffusion was quite limited. Therefore, information with respect to osmosis and diffusion would be processed like a novice in this particular domain. During subsequent information processing, the new information would have to be interpreted by means of the limited prior knowledge in the particular domain or by means of prior knowledge in other domains; links would have to be actively established between the new information and the pre-existing knowledge to extend the limited knowledge in that field. This way of information processing would require cognitive effort and is thus quite divergent from the smooth, almost automatic way of information processing, typical for an expert in a certain domain, which would be induced by schema

II should be pointed out explicitly that from now on schemata are viewed as examples of fixed cognitive structures. So, a schema can reflect the domain-specific knowledge of, for instance, a doctor or refer to knowledge common to almost anybody (e.g., a burglar or homebuyer schema). These concepts are treated identically since they are assumed to instill similar ways of information processing. Chi, Feltovich and Glaser (1981) also proclaimed that experts develop schemata for identifying categories of problems from which solutions were deduced. 
activation. The main aim of this laborious way of information processing is to extend the limited knowledge in that particular area. Therefore, new and important information would be focused during new information processing, which would result in the recall patterns as found in studies supporting the cognitive set-point hypothesis: Overall higher recall scores than a control group, which could largely be attributed to better recall of information that had not been activated previously.

In summary, it is conjectured that in studies supporting the selectiveattention hypothesis information processing did not result in learning in the traditional sense of the word. Prior knowledge was activated by assigning perspectives from which a text should be read, resulting in schema activation. The activated schema would merely have to be up-dated by filling its slots with appropriate information during subsequent information processing. In studies providing support for the cognitive set-point hypothesis, on the other hand, the aim of information processing would be extending the existing prior knowledge; in other words, to learn something new. Activating limited knowledge would therefore result in actively integrating new relevant information into the existing knowledge network in order to extend this knowledge.

\section{RESEARCH GOALS}

The purpose of the research presented in this thesis is to clarify the way in which information processing is influenced by knowledge previously activated. An analysis of the literature resulted in the hypothesis that the nature of the knowledge previously activated could induce different information processes. Activation of highly encapsulated schemata would induce merely updating these knowledge structures, whereas activation of rather limited knowledge for which no ready-made cognitive structure (yet) exists would lead to knowledge extension. In turn, this conjecture could account for the divergent recall patterns as predicted by the selective-attention hypothesis and the cognitive set-point hypothesis. In the case of limited knowledge to start from, the cognitive set-point hypothesis may apply. According to this hypothesis, prior knowledge activation will result in overall higher recall performances. Rellatively less study time would be required to reach the cognitive set-point for information corresponding with the activated knowledge. Consequently, given the condition in which the total amount of study time is fixed, relatively more study time would be available for processing new, nonactivated information. The selective-attention hypothesis predicts better recall of information corresponding with a schema previously activated since most study time would be spent on selecting and processing slot-filling information. Thus, the two hypotheses, both accounting for facilitation effects of prior knowledge activation on subsequent information processing and re- 
call, would not be diametrically opposed; they would merely reflect different ways of information processing, resulting in different recall patterns.

The hypothesis that the nature of the prior knowledge previously activated determines the way of information processing, and thus, the recall pattern will be investigated in a number of experiments, described in the next chapters. Since the (apparently) mutually exclusive results obtained in studies providing support for either the selective-attention hypothesis or the cognitive set-point hypothesis could also have resulted from different operationalizations of prior knowledge activation (e.g., assigning perspectives versus knowledge mobilization), prior knowledge will be activated by means of one technique only: Mobilization. Thus, mobilization is applied to activate generic knowledge, represented in a schema as well as limited knowledge not (yet) represented in a schema.

A preliminary investigation of the postulated hypothesis will be reported in Chapter II. The main goal of this pilot study is to test whether the text constructed met certain requirements. Furthermore, it will be investigated in this pilot study whether a fixed cognitive structure, a schema, as well as knowledge that is not represented yet in such a crystallized cognitive structure can be activated by means of the mobilization technique. Thus, one group of subjects mobilizes all knowledge about a domain that is rather unfamiliar to them for which no ready-made cognitive structure exists. A second group of subjects mobilizes all knowledge they have within a highly familiar domain, resulting in the activation of a "common to anybody" schema similar to the homebuyer or burglar schema (Pichert \& Anderson, 1977).

In Chapter III, the hypothesis that both the selective-attention hypothesis and the cognitive set-point hypothesis may account for facilitation effects emerging from prior knowledge activation on subsequent information processing and recall will be tested in three experiments. From these two hypotheses, predictions can be deducted about the way in which study time is distributed over a text as a result of prior knowledge activation. Therefore, in these experiments in which available study time will be manipulated both recall scores and patterns of study time allocation will be registered.

In Chapters IV and $V$, two more sets of experiments will be reported in which hypotheses are tested, resulting from the findings described in Chapter III. Again, effects of prior knowledge activation on free recall and study time allocation will be presented.

In Chapter $V$, the cognitive set-point hypothesis will be investigated in more detail. The starting point of the cognitive set-point hypothesis is extending the still limited prior knowledge for which no fixed cognitive structure yet exists. This situation is common to most learning. It will be tested whether the predictions of the cognitive set-point hypothesis for recall and allocation of study time also apply for a different type of study material, items 
of categories. If these predictions hold for this more or less isolated study material as well as for a coherent study text, the impact of the cognitive setpoint hypothesis would be enlarged; the assumptions of this hypothesis would also apply to other learning settings.

In the final chapter of this thesis the results obtained will be summarized. Furthermore, conclusions with respect to theories about learning and knowledge representations will be drawn from the reported studies. Finally, suggestions for further research as well as some educational implications will be provided. 
CHAPTER II

\section{INVESTIGATING TWO CONFLICTING HYPOTHESES : PILOT STUDY ${ }^{1}$}

\section{INTRODUCTION}

What a student already knows about a certain topic largely determines the way in which new information is encoded and retrieved from memory (Anderson, 1977, 1978; Brewer \& Nakamura, 1984; Vosniadou \& Brewer, 1987). Ausubel (1960) already indicated that merely possessing prior knowledge is not sufficient. This knowledge should be actively used during information processing to establish relationships between the existing knowledge and the new information. The requirements for effective learning have been met when the student has the disposal of a relevant context in which the new information can be integrated during information processing (Mayer, 1979). In this way, prior knowledge, provided that it is activated, can facilitate information processing. If prior knowledge is not activated, information processing is hampered (Dooling \& Lachman, 1971; Gardner \& Schumacher, 1977).

In Chapter I, it was concluded that prior knowledge activation can facilitate information processing and recall. Two explanations for these effects were provided, the selective-attention hypothesis and the cognitive set-point hypothesis. The predictions derived from these hypotheses differed in the way in which these facilitation effects are manifested.

1. This research has been previously presented in M. Machiels-Bongaerts, H.G. Schmidt and H.P.A. Boshuizen. (1990, April). The Effect of Prior Knowledge Actioation on Text Recall: An Investigation of Two Conflicting Hypotheses. (Report No. TM 015 023). Paper presented at the AERA meeting in Boston, MA. (ERIC Document Reproduction Service No. ED 320925 ). 
It was not yet possible to decide on the respective likelihood of these hypotheses since the studies supporting them differ in operationalization of prior knowledge activation. A closer scrutiny of these studies led to the conjecture that both hypotheses may not necessarily be mutually exclusive. Each of them might apply under different circumstances, depending on the nature of the prior knowledge activated (Schmidt \& Boshuizen, 1992; Shuell, 1990). Activating knowledge represented in different ways would lead to different information processes, which in turn would result in the different recall patterns as predicted by the cognitive set-point and the selective-attention hypothesis.

In this chapter, the assumption is (preliminary) tested whether under appropriate conditions both the cognitive set-point and the selective-attention hypothesis could apply. The predictions of these hypotheses are assumed to be reflected by two experimental conditions. In one condition, a subject group is expected to show the recall pattern predicted by the set-point hypothesis: Better recall of information that had not been activated previously. According to the postulated assumption, this recall pattern would stem from activating knowledge in a certain domain that is quite limited. In order to extend this network, new and relevant information would be focused during information processing. By actively linking the pre-existing knowledge and this new information the initial knowledge could be extended, resulting in better recall of non-mobilized information. In the second condition, represented by a second group, the predictions of the selective-attention hypothesis are tested: Better recall of information corresponding with the activated knowledge. It was hypothesized that this recall pattern would emerge if the activated prior knowledge was represented in a ready-made cognitive structure. During subsequent text processing, merely information would have to be selected that filled slots of this schema with appropriate values. Since most attention would be paid to slot-filling information, this information would be recalled best.

These two conditions reflecting the two apparently mutually exclusive hypotheses were tested in a (pilot) study. The first group, the EEC group, was assumed to confirm the predictions of the cognitive set-point hypothesis. The EEC subjects had to mobilize all their knowledge about the EEC fishery policies, which was very much "in the news" at the time of testing (e.g.r protection of fish stock, fishery quota, penalties); subjects' knowledge in this domain was expected to be quite limited. The tourism group was expected to show the predictions of the selective-attention hypothesis. This group had to mobilize general factors that could be important for a tour operator who had to select new holiday locations (e.g., climate, surroundings, recreational activities); it was assumed that this mobilization strategy would result in the activation of a "common to everyone" schema.

To study the information processes of these two groups, representing the two hypotheses, the study text had to meet a number of requirements. First, 
the text had to contain information corresponding with the EEC group's limited prior knowledge, the so-called EEC information. Since during mobilization the EEC group quasi started processing this information, the cognitive set-point hypothesis assumes that little attention would have to be paid to this information to reach the cognitive set-point. Second, the set-point hypothesis predicts that the EEC subjects would try to extend their limited knowledge by actively integrating new relevant information; consequently, the text must contain information that could be related to the EEC group's prior knowledge, the so-called fish-farm information. This information category would be focused during subsequent information processing, resulting in better recall of this information. Third, the text had to comprise information filling the slots of the schema activated by the tourism group. Thus, the text had to provide specific information ("couleur locale") about an imaginary village, the socalled tourism information. During subsequent information processing, the tourism group was expected to select this information. Information that did not fill slots of the schema activated would be ignored, irrespective of its importance in the text itself. The selection process, stemming from schema activation, would be demonstrated more clearly if the slot-filling information would be rather redundant in the text itself.

To test whether the text indeed matched the prior knowledge activated, subjects had to mark text elements that were important with respect to their instruction during text processing. The EEC information was assumed to be relevant for the EEC group and the tourism information for the tourism group. This task was designed to test whether mobilization was an effective strategy to activate prior knowledge.

\section{PILOT STUDY}

The goal of this pilot study was twofold. First, it was tested whether by means of one technique both generic knowledge, represented in a schema and limited specific knowledge, that is not represented in a schema can be activated. Second, it was tested whether the text indeed contained information matching the prior knowledge of the two groups.

\section{MATERIAL}

To test these assumptions a text was developed, entitled "The consequences of the EEC fishery policies for Crando Douro" (Appendix I). This text was divided into propositions, resulting in a text base. According to Schmidt (1982a), propositions can be defined as "... combinations of subject and predicate, expressing one idea. Propositions can be identified in texts by marking 
conjunctions, adverbs, relative pronouns, periods and semi-colons. (...) Sometimes propositions are separated by comma's or brackets" (p. 40). The following example, showing the first text part, will illustrate this technique.

Since 1986 the EEC fishery policies are in force for Portugal too, / which led to an unemployment rise in many small fishing-villages. / Due to the fish catch limitations the income dropped, / (which increased unemployment.) $)^{1}$

The text's hierarchical structure, presented in Appendix $\mathrm{II}_{r}$ is based on Meyrer's (1975) system, which is comparable with Grimes' (1975) model to describe the organization of a text. The text's content structure is depicted by a so-called tree, showing the way in which text parts are linked (co-ordinate or subordinate). The text content is hierarchically arranged; the most important information appears in the top of the tree.

The text, consisting of 1372 words, contained 57 sentences. ${ }^{2}$ Four different information categories could be identified in this text:

\section{a. EEC information}

This text passage contains information about the backgrounds of the EEC fishery policies, which had immense consequences for the imaginary fishing-village in Portugal. For instance, unemployment rose due to the EEC measures. This important information category (mean level of 4.23 in the text hierarchy) was expected to correspond with the EEC group's prior knowledge. The EEC information, divided into 22 propositions, consisted of ten sentences, which contained approximately equal numbers of words, syilables and characters.

b. Fish-farm information

In this text passage an alternative income source is pointed out to resolve the unemployment in the Portuguese fishing-village, resulting from the EEC policies. This information category could be related to the EEC infor-

1 This text part is placed between brackets since it is identical to the second propiosition. It is not considered a new proposition but merely a paraphrazed repetition of the second propo-
sition.

2 The original text was written in Dutch since Dutch students participated in the studies reported. It was strived for to construct information parts approximately equal in length (numbers of words, syllables and characters). Naturally, the text's translation resulted in deviant numbers. Appendix II contains, besides the hierarchical text structure, the mean numbers of words, syllables and characters for the EEC information, the fish-farm information and the tourism information, referring to the original Dutch version. 
mation. The fish-farm information was completely fictitious; so it could be assumed that it was new and unknown to all subjects. This information was as important as the EEC information in the text (mean level of 4.23 in the text hierarchy). This category also consisted of ten sentences or 22 propositions.

c. Tourism information

This information category concerned rather tourist information about the village in question ("couleur locale"). It contained descriptions of the population, surroundings, traditions, ways to relax, climate, et cetera. This information was considered to correspond with slots of the schema previously activated by the tourism group. In the text itself the tourism information was relatively unimportant; therefore, it occupied at a rather low level in the hierarchical text structure (mean level of 6.63). This information category also consisted of ten sentences, which could be divided into 22 propositions. The sentences of the tourism information were scattered all over the text. Selective attention would be particularly demonstrated since (a) the slot-filling information had to be gathered from all over the text, and (b) the slot-filling information played an inferior role in the text itself. ${ }^{1}$

d. Residual information

In order to build a coherent text 27 (short) filler sentences were added to the three information categories. These sentences could be divided into 34 propositions (mean level of 5.17 in the text hierarchy).

\section{SUBJECTS}

In this study, 20 subjects participated, all students at the University of Limburg, who were randomly assigned to the EEC group or the tourism group. Their average age amounted to 19 years and 5 months (ranging from 18 to 23 years). For participating, they received a financial compensation.

\section{PROCEDURE}

This pilot study consisted of four elements which each subject carried out individually. The experiment was conducted in a separate room to prevent noise

\footnotetext{
1 Normally, the text structure, showing the importance of the various parts of information, determines which information category is recalled best. Important information (i.e. on a high level in the text structure) is recalled better than less important (low-level) information. The expression "levels effect" refers to this phenomenon (Britton, Meyer, Simpson, Holdredge, \& Curry, 1979; Meyer, 1975; Meyer, Brandt \& Bluth, 1980; Meyer \& McConkie, 1973; Meyer \& Rice, 1984; Thomdyke, 1977). Additional information with respect to the lev els effect is provided by Hustinx (1985).
} 
pollution as much as possible, which could break the subject's concentration while mastering the tasks.

The EEC subjects were told that lately much attention is paid to the EEC fishery policies in the media. They were requested to tell everything they knew about the EEC fishery policies. It was expected that information like overfishing, protection of fish stock, protection of fishery industry, quota and penalties by the government (General Inspection Service) would be activated. If the initial question did not induce these aspects, additional questions were asked to still activate this knowledge, like "What are the functions of the EEC fishery policies?", "What would happen to the fish stock if no fishery policies existed?", "In what way are the EEC regulations preserved?", et cetera. By means of this task, the EEC group orally mobilized knowledge that was assumed to be relevant to the EEC information in the text. The tourism group was requested to mention as many factors as possible that would be of importance for a tour operator who had to decide whether a location would be apt as a new holiday resort. Subjects had to keep in mind that a tour operator's clients differ in preferences, so they should not only mention personal criteria. They should indicate the impact of each factor per category of clients. It was assumed that this instruction would activate factors like climate, costs, recreational activities, surroundings and atmosphere. If subjects failed to mobilize one of these factors, which rarely happened, this factor was used as key word. By presenting this key word, subjects had to describe the factor's impact on the various categories of clients. By means of this task, a holiday schema would be activated. During the study phase, the schema slots could be filled with tourism information. Prior knowledge mobilized by each subject was recorded on tape.

After mobilizing prior knowledge, each subject in the EEC group and the tourism group read once the same text, entitled "The consequences of the EEC fishery policies for Crando Douro" (see Appendix I). In order to check whether the prior knowledge mobilized by the EEC group and the tourism group corresponded with the EEC information and the tourism information respectively, all subjects were instructed to mark while studying the text all elements that were important in light of their instruction. Thus, this task was designed to explore whether the mobilization technique was an effective strategy to activate prior knowledge that was either represented in an associative network or in a crystallized cognitive structure. Subjects were not limited in the amount of time required to carry out this task. The text could be studied just once. Reading the text and marking all relevant text elements took approximately ten minutes. In this respect, the two groups of subjects hardly
differed.

Subsequently, to divert the subjects from the literal wordings of the text, a number of (medical) questions had to be answered in writing (e.g." "Describe 
as many embolism processes as possible taking into account the relationship between the clinical manifestations and anatomy or physiology"). During 20 minutes, a maximum of four questions had to be answered. Keenan and Kintsch (1974) showed that such a distraction task clears the literal wordings of a text from memory.

A free recall test completed the experiment. Subjects were asked to recall orally everything they could remember from the text. Subjects" free recall was recorded on tape.

\section{RESULTS}

The recall protocols were literally transcribed. For each category of information, both the number of propositions marked and the number of propositions correctly recalled were calculated for each subject.

In order to estimate the reliability of this procedure, 6 of the 20 recall protocols were randomly selected. Two raters scored the number of correspondences (literal or paraphrazed) between the text base and each recall protocol. The mean interrater agreement was $88 \%$ (ranging from $82 \%$ to $91 \%$ ). Since this sample resulted in a relatively high interrater agreement, it was presumed that the two raters would agree to the same extent for the remaining 14 protocols.

The results of the two subject groups were compared by means of (twoway) analyses of variance. Subsequently, Newman-Keuls comparisons were used to compare the two groups' scores for each information category. T-tests were applied to examine whether each group differed in numbers of propositions marked or recalled per information category. To permit comparisons between the four information categories, the scores for residual information were transformed to a scale with a minimum of 0 and a maximum of 22 since this information category originally consisted of more propositions than the three remaining categories.

To test whether the text met the requirements, subjects had to mark relevant information during text processing. The EEC group mobilized knowledge about the functions and consequences of the EEC fishery policies. Therefore, this group was assumed to mainly mark EEC information. The tourism group was expected to mainly mark tourism information since this information would fill slots of the activated schema. Table 2.1 presents per information category the mean numbers of propositions marked by the two groups during reading.

The two groups of subjects differed in the total number of propositions marked while processing the text $\left(F(1,18)=7.614, p<.05, M S_{e}=9.54\right)$. The numbers of propositions marked per information category differed, $F(3,54)=$ $47.703, p<.0001, M S_{e}=2.47$. Furthermore, the interaction of treatment and 
information category appeared to be significant, $F(3,54)=201.768, p<.0001$. $M S_{e}=2.47$; in other words, the number of propositions marked per information category was not identicall for the two groups.

Table 2.1

Mean number of propositions per information category marked during text studying as a function of mobilization treatment. The figures between brackets refer to the standard deviations.

Information category

$\begin{array}{lccccc}\begin{array}{l}\text { Mobilization } \\ \text { treatment }\end{array} & \text { EEC } & \text { Fish-farm } & \text { Tourism } & \text { Residual* } & \text { Totals } \\ \text { EEC group } & 12.2(2.9) & 4.1(3.1) & 2.1(2.2) & 7.0(1.5) & 25.4(8.2) \\ \text { Tourism group } & 0.2(0.4) & 0.4(1.0) & 14.0(2.2) & 3.2(1.7) & 17.8(3.1)\end{array}$

* Scores for residual information (34 propositions) have been transformed.

Newman-Keuls comparisons (alpha set at .01) showed that the EEC group marked more propositions of each information category than the tourism group; except for tourism information, the latter group showed higher scores.

The EEC group mainly marked $\mathbb{E E C}$ information during reading. The number of propositions marked of this category exceeded the numbers of propositions marked of the fish-farm information $(t(9)=7.727, p<.0001)$, the tourism information $(t(9)=14.982, p<.0001)$ or the residual information $(t(9)$ $=8.711, p<.0001) .1$ The tourism information was most relevant for the tourism group since of this category the largest number of propositions had been marked. This number exceeded the numbers of propositions marked of the EEC information $(t(9)=18.225, p<.0001)$, the fish-farm information $(t(9)=$ $20.299, p<.0001$ ) or the residual information $(t(9)=12.099, p<.0001) .^{2}$

In order to produce a (prelliminary) reply to the question whether both the cognitive set-point and the selective-attention hypothesis could account for the facilitation effects emerging from prior knowledge activation, the recall scores of the two groups were compared, which are presented in Table 2.2 .

1 The EEC group also marked more propositions of the residual information than of the fishfarm information $(t(9)=4.061, p<.01)$ or the tourism information $(t(9)=10.088, p<.0001)$.

2 The tourism group also marked more propositions of the residual information than of the EEC information $(t(9)=5.524, p<.001$ ) or the fish-farm information $(t(9)=5.56, p<.001)$. 
Table 2.2

Mean number of propositions per information category correctly recalled as a function of mobilization treatment. The figures between brackets refer to the standard deviations.

Information category

\begin{tabular}{lccccc}
$\begin{array}{l}\text { Mobilization } \\
\text { treatment }\end{array}$ & EEC & Fish-farm & Tourism & Residual & Totals \\
\hline EEC group & $12.0(5.3)$ & $16.8(4.9)$ & $5.9(3.6)$ & $10.9(3.4)$ & $45.6(11.8)$ \\
Tourism group & $1.9(2.7)$ & $3.8(2.3)$ & $12.9(4.5)$ & $5.1(2.2)$ & $23.7(9.8)$
\end{tabular}

* Recall scores for residual information (34 propositions) have been transformed.

The two groups differed in the total recall scores, $F(1,18)=20.475, p<.001$, $M S_{e}=29.4$. Different numbers of propositions were recalled per information category $\left(F(3,54)=4.768, p<.01, M S_{e}=9.25\right)$. Furthermore, a significant interaction of the factors mobilization treatment and information category was found, $F(3,54)=42.138, p<.0001, M S_{e}=9.25$.

Newman-Keuls comparisons (at the .01 level) showed that the EEC group outperformed the tourism group in recall of each category, except for tourism information; in this respect, the latter group obtained higher recall scores.

The EEC group excelled in recall of fish-farm information. This recall score was significantly higher than the recall scores obtained for EEC information $(t(9)=3.398, p<.01)$, tourism information $(t(9)=6.17, p<.0001)$ or residual information $(t(9)=3.128, p<.01)$. Although the EEC group had mainly marked $E E C$ information, the non-activated fish-farm information was recalled best. The tourism group, on the other hand, excelled in recall of tourism information. This score exceeded the recall scores for EEC information $(t(9)=10.436, p<.0001)$, fish-farm information $(t(9)=8.957, p<.0001)$ or residual information $(t(9)=6.753, p<.0001$ ).

The numbers of propositions marked and recalled by the EEC group for each information category are presented in Figure 2.1.

Figure 2.1 illustrates that the EEC group mainly recalled new important fish-farm information. The number of propositions of the fish-farm information marked during reading was only 4.1; for recall, however, this number quadrupled (16.8). Apparently, this new relevant information had been focused during information processing, although mainly EEC information matching the activated knowledge had been marked. By integrating the important fish-farm information in the prior knowledge activated, this network 
could become extended. Despite this increase in recall of fish-farm information $(t(9)=7.918, p<.0001$ ), recall of EEC information was not impaired as the number of propositions recalled was almost equal to the number of propositions marked $(t(9)<1)$. Furthermore, the EEC group showed an increase in recall of both tourism information $(t(9)=7.425, p<.01$ ) and residual information $(t(9)=8.043, p<.01)$, compared to the number of propositions marked.

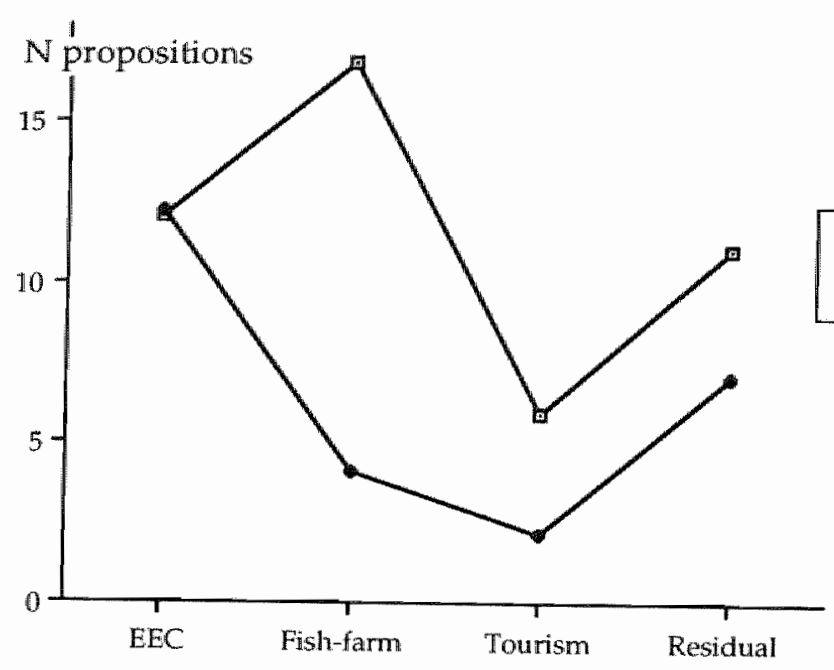

Information category

Figure 2.1 Mean number of propositions per information category marked and recalled by the EEC group

Figure 2.2 illustrates that the tourism group did not show such a large rise in recall, compared to the numbers of propositions marked. This group appeared to retain to text elements that filled slots of the activated schema. For this tourism information, the number of propositions marked did not differ from the number of propositions recalled $(t(9)<1)$. With respect to recall of the remaining information categories, no differences were expected compared to the number of propositions marked during reading. For the tourism group, the number of propositions marked during reading and the number of propositions recalled was almost identical for the EEC information $(t(9)=1.899, p=$ .09). A slight rise in recall was found for residual information, but the difference between the numbers of propositions marked and recalled did not reach the significance threshold $(t(9)=2.144, p=.06$ ). Significant differences in this respect were only found for the fish-farm information $(t(9)=4.456, p<.01$ ). 


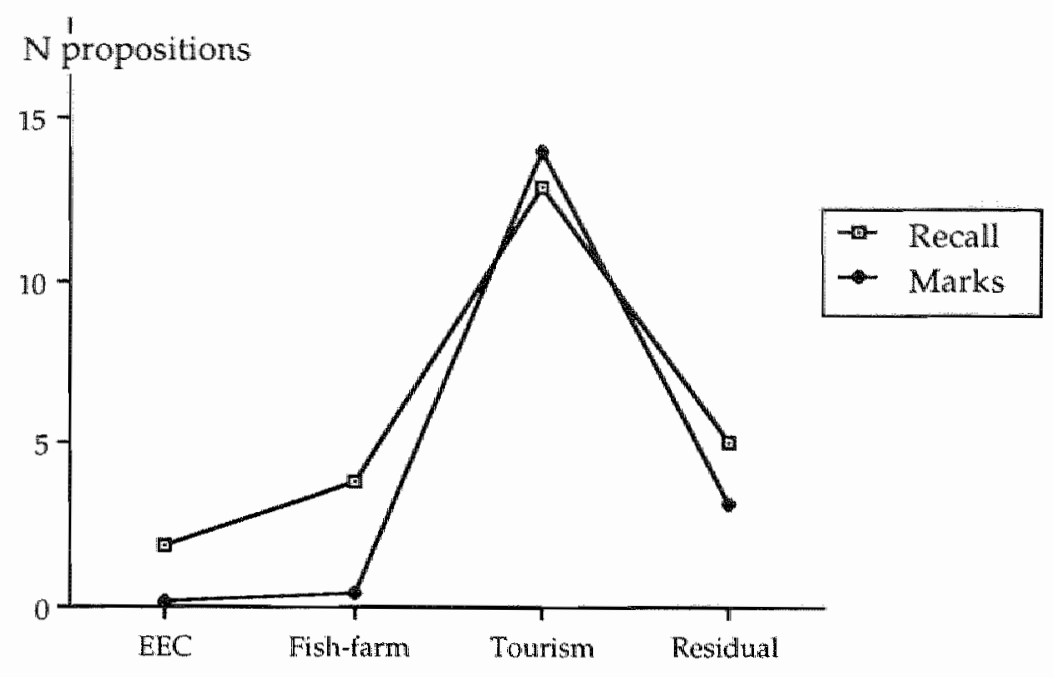

Information category

Figure 2.2 Mean numbers of propositions for each information category marked and recalled by the tourism group

GENERAL DISCUSSION

The results of this pilot study suggest that both the selective-attention and the cognitive set-point hypothesis may account for the facilitation effects emerging from prior knowledge activation. The tourism group and the EEC group showed different recall patterns, corresponding with the predictions of the selective-attention and the cognitive set-point hypothesis respectively. The tourism group excelled in recall of tourism information; all the other information categories, which were more important in the text itself, were recalled worse. According to the selective-attention hypothesis, this recall pattern resulted from the information process induced by schema activation. The tourism group had mobilized knowledge that was assumed to be represented in a crystallized cognitive structure. This schema would direct the tourism subjects to select tourism information, filling the slots of this schema. The remaining information did not meet this criterion, which would be largely ignored. Consequently, this information could not be retrieved from memory during free recall. This would explain why schema activation results in better recall of information corresponding with the knowledge previously activated.

Compared to the number of propositions marked during reading, the EEC group showed a huge increase in the number of propositions recalled of the fish-farm information which had not been activated previously. This rise 
in recall of important new information did not result in a deterioration of previously activated EEC imformation since the number of propositions recalled equaled the number of propositions marked. The EEC group's recall pattern captures the predictions of the cognitive set-point hypothesis. Activating prior knowledge would lead to a reduction of the amount of study time required to reach the set-point of information corresponding with this knowledge. Relatively more study time would remain to process new non-activated information. By integrating this new information, the original limited knowledge could become extended. Therefore, an increase in overall recall was predicted, as was shown by the EEC group. Strikingly, the predicted rise in recall did not only emerge for the new important fish-farm information, but also for residual information and tourism information. Apparently, enough time was left that could be spent on these less important information categories, resulting in an increase in recall for these categories as well.1

The results obtained in the pilot study demonstrated that mobilization appears to be an appropriate technique to activate generic prior knowledge represented in a schema or limited specific knowledge not represented in such a ready-made cognitive structure. The text constructed indeed contained information categories that largely corresponded with the knowledge previously activated by the two groups. The EEC group had mainly marked EEC information, that was designed to correspond with the knowledge previously mobilized by this group. The tourism group, on the other hand, had mainly marked tourism information while text processing; this information was expected to fill slots of the schema previously activated by the tourism group.

However, based on these results no ultimate statements can be made about the hypothesis postulated, i.e., that the selective-attention hypothesis and the cognitive set-point hypothesis only appear to be diametrically opposed, since they were obtained by means of a paradigm (marking relevant information during reading) that did not allow an on-line observation of the information processes as predicted by the two hypotheses under study. These predictions could be tested more adequately if the amounts of study time spent on the various information categories would be registered. From the assumptions of the two hypotheses, predictions about patterns of study time allocation can be deduced. However, in this study the amount of total time required to study the text had not been registered precisely. Furthermore, data about the way in which study time was distributed over the four information categories of the text is lacking. These shortcomings will be provided for in
Chapter III.

1 Even more strikingly, the EEC group did not show a rise in recall of EEC information, compared to the number of propositions marked. This information was, apart from the fishfarm information, the most important information category in the text. Yet, the EEC group's
recall score was merely 12.0 (maximum recall score per information category was 22). 
CHAPTER III

\title{
EFFECTS OF PRIOR KNOWLEDGE ACTIVATION ON STUDY TIME ALLOCATION AND FREE RECALL ${ }^{1}$
}

\begin{abstract}
Effects of mobilizing prior knowledge on information processing were studied. The cognitive set-point and the selective-attention hypothesis predict different patterns of recall and study time allocation as a result of mobilizing prior knowledge. It is hypothesized that these cognitive frameworks are not mutually exclusive, but that they may apply to different learning situations. The selective-attention hypothesis would apply to situations where the subject already possesses an extended knowledge base, represented in a schema. Schema activation would lead to up-dating this ready-made cognitive structure during information processing. The cognitive set-point hypothesis may apply when no such elaborated preconceived framework (yet) exists; knowledge extension would then be the goal of text processing. These different information processes would result in different recall patterns.

The assumption that both hypotheses may apply were tested in three studies. Available study time was manipulated since different predictions regarding patterns of study time and recall can be derived from the two hypotheses.
\end{abstract}

1 Parts of the research reported have been previously presented in M. Machiels-Bongaerts, H.G. Schmidt and H.P.A. Boshuizen, (1991, April). The Effects of Prior Knowledge Activation on Free Recall and Study Time Allocation. (Report No. TM 019 127). Paper presented at the AERA meeting in Chicago, IL. (ERIC Document Reproduction Service No. ED 350 349). A. copy of this chapter has been submitted for publication under the title "Selective-Attention versus Cognitive Set-Point Hypothesis in Text Processing: Studies on Free Recall and Allocation of Study Time' by M. Machiels-Bongaerts, H.G. Schmidt and H.P.A. Boshuizen. 
The results show that mobilizing prior knowledge can result in different patterns of recall and study time allocation, supporting the assumption that both the cognitive set-point hypothesis and the selective-attention hypothesis may apply, depending on the nature of the prior knowledge previously mobilized.

\section{INTRODUCTION}

The past fifteen years, the study of text comprehension has been dominated by the schema-theoretic approach (Anderson, 1977; Rumelhart \& Ortony, 1977; Schank \& Abelson, 1977). Reading a text is assumed to activate a relevant schema, which, in turn, guides text processing in a top-down fashion. A schema can be described as a cognitive structure, containing information abstracted from situations or events frequently experienced (Anderson, 1990). These knowledge structures contain variables (or, slots or placeholders) in which information from real world experiences or from their textual representations can be inserted. Comprehension is, therefore, in the schema-theoretic view primarily a process of instantiating an appropriate schema.

Take, for instance, a Dutch tourist planning a holiday in the US and reading the Wall Street Journal about a newly developed holiday resort in Florida. It is assumed that this tourist will activate a tourism schema and particularly process information fitting into that schema. He will pay attention to the weather conditions in the area, the availability of beaches and other tourist attractions, hotel facilities and the US dollar's current value. This information is attended to and stored into what becomes an instantiation of the tourism schema. A Dutch investor, on the other hand, may pay attention to quite different aspects of the same article. He may be interested in growth potential of the new development, the organization's cash-flow position, the quality of the management and the US interest rates. In the investor's case, an investment schema is activated in which the new information is stored. This instantiation may form the basis of future investment decisions.

Schema theory assumes that during information processing a distinction is made between information relevant to the active schema and irrelevant information, which would largely be discarded (Anderson, 1982). This process of selective attention would lead to better recall of information corresponding with the schema and relatively poor recall of schema-irrelevant information. The seminal perspective-taking studies of the Center for the Study of Reading at the University of Illinois (Anderson \& Pichert, 1978; Pichert \& Anderson, 1977) were based on precisely this assumption. Pichert and Anderson (1977), for instance, required subjects to read a story about a house and its contents from one of two perspectives: A burglar or a homebuyer perspective. Subjects mainly recalled information relevant to the perspective previously ascribed. This differential recall phenomenon is assumed to emerge because subjects 
spend more attention, expressed as more study time, on the relevant text parts. Indeed, Goetz. et al (1983) showed that 20 to $50 \%$ more time was spent on sentences relevant to the active schema than on irrelevant sentences. Hence, the instruction to take a particular perspective seems to activate appropriate prior knowledge, leading subjects to selectively attend to slot-filling information. This, in turn, results in better recall of information corresponding with the prior knowledge activated (Alba \& Hasher, 1983).

But what if the subject does not have a specific relevant schema available for text processing? Imagine a first-year Dutch economy student who uses the Wall Street Journal article to acquire an initial understanding of US economy. He may have some general notions about economy available to guide him through the text but his recall may be quite different from the tourist's or the investor's recall. In the student's case, comprehension may not result in instantiating and up-dating an already existing schema simply because no such schema exists. The question is, then, what happens when no readymade schema is available for comprehending new information ${ }^{1}{ }^{1}$ It will be demonstrated in this chapter that, when pre-existing schemata are not (yet) available, the reader tries to extend his knowledge base rather than up-dating it. Thus, schema activation would lead to selecting information corresponding with this knowledge, whereas activating limited prior knowledge would result in focusing new, important information during subsequent text processing in order to extend the initial prior knowledge.

Evidence for this kind of extension of a knowledge base beyond what is already known was found in a study by Peeck et al. (1982). They required primary-school children to write down their knowledge of foxes, their appearances and habits. Subsequently, these children received a text describing a fictitious animal, the American heath fox. The text contained not only information in line with the knowledge previously activated (or "mobilized"as Peeck et al. call it), but also information that was in variance with this knowledge, and information considered new but not necessarily conflicting with the children's prior knowledge. As to be expected, Peeck et al. (1982) found a facilitative effect of knowledge mobilization on text recall. Contrary to what the selective-attention hypothesis would predict, however, the facilitative effect was mainly found for information not mobilized previously. In other words, the experimental group excelled over the control group mainly in recall of information not previously activated. A similar phenomenon was found by Schmidt (1982b). He required secondary school children to mobilize their knowledge about the cell-biological processes of osmosis and diffusion before administering a text on these topics. The text in addition contained informa-

1 Those situations are quite common in most natural learning situations since the very idea of schooling is that students learn things that may be entirely or largely new to them. Schema theory cannot easily accommodate these situations. 
tion about related topics such as assimilation, the structure of cells, turgor and plasmolysis. His experimental group outperformed the control condition mainly with regard to the latter information, that is: Information not mobilized prior to text processing. Similar findings are reported by Peeck (1982).

In an attempt to explain why the experimental groups in the studies of Peeck et al. (1982), Peeck (1982) and Schmidt (1982b) performed better particullarly on recall of information for which no activation previously took place, Schmidt (1983b) proposed a cognitive set-point hypothesis. According to Schmidt, readers apply an internal standard or set-point helping them to decide when sufficient time has been allocated to a certain piece of information. The set-point level is supposed to be determined by an estimation of some minimum amount of understanding by the reader, the reader's goals, his motivation, and external pressures such as the expected consequences of not being able to reproduce adequately the information. If the set-point for a particular information unit has been reached, the reader will proceed to process new information, until the set-point for all information units in the text has been reached or time has run out. ${ }^{1}$ Schmidt assumes that, when subjects activate prior knowledge previous to reading a text, they already process this information to a certain extent. When they, subsequently, encounter the same or similar information in the text, they need less time to reach the set-point for that information. If the total time for text processing is restricted, as was the case for all studies referred to, mobilization groups will have more time available to process non-activated information relative to a control condition; hence, better recall performance on this part of the information.

This theory allows for a number of predictions. Imagine a series of studies in which a text is sequentially presented on a computer screen under varying processing conditions. The experimental group is required to mobilize prior knowledge with regard to a part of the text, and processing time per information unit can be measured or manipulated. If total study time is restricted (but time to study individual information units is free), one would expect the mobilization group to spend less time on information previously mobilized, relative to a control group, and more time on non-mobilized information. In addition, one would expect better overall recall by the experimental group but in particular for the non-mobilized information. This would occur because the experimental group would have more time available than a control group to reach the set-point for that part of the text as well. If total study time, however, would be free, the mobilization group would show no better recall to a control group since both groups are in a position to reach the set-point for all information units. The experimental group, however, would require and, therefore, use less time to process the information previously ac1 'This hypothesis accounts for the obvious observation that readers do not infinitely prom
ceed processing the same infornation but move on through the text as they study' it. 
tivated. In a third study, in which the information is presented with a fixed rate, processing times, of course, will not differ. Moreover, no recall differences would arise. The experimental group would no longer be able to benefit from its processing advantage stemming from mobilizing relevant prior knowledge since time spent on information previously mobilized could not be reduced nor could more time be spent on non-mobilized information.

There is scant evidence supporting these hypotheses. The studies conducted by Peeck et al. (1982) and Schmidt (1982b) were already mentioned. While total study time was restricted, they found the predicted recall patterns: The mobilization group outperformed the control group in recall of nonmobilized information. A study by Johnson and Kieras (1983) appears to support the set-point hypothesis under the free study time condition. In that study, subjects were confronted twice with short texts. The sentences of each text were presented one by one on a computer screen. During the first presentation, subjects had to indicate whether the particular sentence contained information previously known; the second time, they had to study each sentence in order to recall it. Study times tended to be shorter for sentences containing information that subjects had rated as previously known. No recall differences, however, were found between this group and a control group, as is predicted by the cognitive set-point hypothesis. Since total study time was free, both groups had the opportunity to reach the set-point for all information units. Additional support comes from list processing studies. Peeck (1982) required subjects to mobilize names of US presidents or states. The subjects who mobilized presidents performed better in this category while subjects mobilizing states showed better recall of state names. Peeck, however, found that the differences between the experimental groups and the control group emerged mainly for information not previously mobilized. (He did not measure processing times). A study by Machiels-Bongaerts, Schmidt and Boshuizen (in press), replicating and extending Peeck's (1982) findings, found recall and study time patterns supporting the cognitive set-point hypothesis.

The issue here is that the predictions of the set-point hypothesis seem to be diametrically opposed to those of the selective-attention framework. For instance, for the imaginary experiment in which the experimental group would mobilize prior knowledge with regard to a part of the text (much in the same way as prior knowledge was activated in the Pichert and Anderson's (1977) study) and total study time would be restricted, the selective-altention hypothesis predicts better recall of information previously activated and longer study times of that information, whereas the cognitive set-point hypothes is predicts quite the opposite. The question, then, is: How come that these two hypotheses regarding the nature of information processing produce such different predictions? A possible answer is provided by Machiels-Bongaerts 
(1993). She assumes that these two descriptions of how people process texts in fact apply to two different learning situations: The selective-attention hypothesis would apply to those situations where the subject already has an extended knowledge base tightly knit together as a prototypic schema for a known class of situations and events. The prospective tourist and the investor reading the Wall Street Journal article are obvious examples. They have well-formed schemata available for text processing and tend to ignore information irrelevant to their perspective. Acquiring new information in these cases is more a matter of knowledge up-dating than of learning. The same may apply to the processing task of the subjects in the burglar/homebuyer studies (Anderson \& Pichert, 1978; Goetz et al., 1983; Pichert \& Anderson, 1977). These subjects activated an already existing schema and up-dated their knowledge with the facts provided in the text. The learning task for someone such as the Dutch economy student trying to understand US economy is, however, quite different. Here, a subject with rather limited knowledge in that domain, without any preconceived and well-tested schema, is trying to make sense out of a text. He may have some notions relevant to the subject-matter dealt with in the article, but these notions do not constitute a prototypic schema. They are rather the primitives that may be used to construct a more or less coherent text representation. Under these conditions, knowledge extension rather than knowledge up-dating is the goal of text processing. This may be the situation to which the set-point hypothesis would apply. Subjects process information to a set-point level of comprehension and then move on to new information, which does not necessarily need to fit into a preconceived framework to be processed. In fact, this new information may not easily fit within such framework, simply because a framework does not yet exist. In the General. Discussion we will elaborate on these notions within the broader perspective of Kintsch's (1988) theory of text processing.

Here, our aim is to report on three studies designed to pursue two goals. First, the effects of prior knowledge mobilization on information processing were studied. To what extent can the cognitive set-point hypothesis explain patterns of study time allocation and free recall under various processing conditions? Second, we were interested in the extent to which the selectiveattention and the cognitive set-point hypothesis apply, given appropriate conditions. To that end, a text was constructed containing both information for which the subjects were expected not to have any elaborated preconceived schema and information of which the subjects had schematic knowledge. Prior to studying the text, subjects mobilized prior knowledge relevant to the non-schematic information in the text or to the schematic information. It was expected that subjects would either show patterns of recall and study time allocation predicted by the cognitive set-point or the selective-altention hypothesis, depending on the nature of the experimental manipulation. The 
experiments were designed in the way described previously: Study times and free recall were measured. Time available to study individual information units was manipulated in the three studies. A distinction was made between total study time, that is: The amount of time available or used to study the text as a whole, and local study time, that is: Time available or used to study single units of the text, mostly sentences. In Experiment 1 , total study time was fixed whereas subjects were free to allocate time to the various text parts. In Experiment 2, both total and local study time were free. In Experiment 3, both local and total study time were fixed. These manipulations of available study time enabled us to study in detail the predictions of the two hypotheses.

EXPERIMENT 1

This study was conducted as a first test of the assumption that both the cognitive set-point and the selective-attention hypothesis may provide accurate explanations for text processing phenomena, provided that specific conditions regarding the nature of the reader's prior knowledge are met. To that end, a text was constructed describing the effects of the European Economic Community's restrictive fishery policies on a small imaginary fishermen's village in Portugal. The text outlines these policies, including limited fishing quota for member countries, and describes their effects on the village's faltering economy. The text proceeds with a possible way of dealing with the consequences of these policies: Establishing a fish-farm. Throughout the text references are made to the natural beauty of the village and its potential as a future holiday resort. The EEC fishery policies were in the news quite often when the present studies were carried out. Possibly because of that, subjects were able to generate quite some knowledge on the subject-matter in a pilot study (Machiels-Bongaerts et al., 1991), however, without showing any signs that this knowledge already had a schematic structure of any kind. ${ }^{1}$ Regarding the idea of establishing a fish-farm, subjects had no knowledge at all, simply because this information was fabricated by the experimenters.

In the first study, total study time was fixed but could be freely distributed over the various text parts, in a way similar to the original studies by Peeck (1982), Peeck et al. (1982) and Schmidt (1982b), which gave rise to the cognitive set-point hypothesis. Three subject groups participated in the ex-

\footnotetext{
1 This type of knowledge can perhaps be better characterized as a set of concepts loosely connected in an associative network; the links between concepts having low association strengths (Kintsch, 1988). Contrary to the knowledge organized in a schematic fashion, this kind of knowledge quickly becomes inaccessible as time passes. Some time after the news papers stopped writing about the EEC fishery restrictions in the Netherlands, follow-up studies were conducted in which the same text was applied. Subjects were only able to generate a fraction of the knowledge available in the same population two years before.
} 
periment. One group activated knowledge about EEC fishery policies; a second group activated (schematic) knowledge on what is important for tourism; a third, control, group mobilized knowledge about an unrelated topic. It was predicted that the EEC group would behave in accordance with the set-point hypothesis, whereas the tourism group would show a study time and recall pattern in line with the selective-attention framework. The EEC subjects would spend relatively less time on EEC policy information because they already process that information during mobilization and therefore need less time to reach the set-point for that information. It was also predicted that the EEC group would spend more time on a possible solution for the consequences of these policies for the Portuguese village (the fish-farm information', because, compared to the control group, the EEC group has more time available to do so. Recall patterns would be in agreement with the study time patterns. By contrast, the tourism group would selectively attend to information relevant to that perspective. It was predicted that this group would spend more time on tourism information than the other groups and show better recall of this information (Goetz et al., 1983; Pichert \& Anderson, 1977).

\section{SUBJECTS}

In Experiment 1, 18 subjects participated, randomly assigned to one of the three conditions: The EEC group, the tourism group or a control group. The subjects' average age was 19 years and 9 months (ranging from 18 to 23 years). Subjects were all undergraduate students of economy at the University of Limburg. For participating, they received a financial compensation.

\section{PROCEDURE}

Subjects were run individually. The EEC subjects had to generate all their knowledge about EEC fishery policies. The tourism group had to generate all knowledge of general factors that could help a tour operator to decide whether a location is suited to become a new holiday destination. The control group mobilized knowledge about tennis, which was in no way related to the study text. Mobilization, lasting for about five minutes, was recorded on tape.

Subsequently, each subject studied the text "The consequences of the EEC fishery policies for Crando Douro" (Appendix I), describing the actions and consequences of the EEC fishery policies for a small, imaginary fishingvillage in Portugal. ${ }^{1}$ As has been explained, the text was constructed such

\footnotetext{
1 An imaginary village was depicted to control for possible specific experiences of the subjects with the village; yet, some subjects claimed that they had visited Crando Douro.
} 
that it contained information relevant from the EEC perspective and information relevant from the tourism perspective. It contained three information sets, each consisting of ten sentences or 22 propositions. The EEC information corresponded with the prior knowledge previously mobilized by the EEC group. The second information set, the fish-farm information, was new to all subjects. The third set, the tourism information, was intended to match the tourism group's schematic knowledge of this topic. A pilot study showed that the text met these requirements (Machiels-Bongaerts et al., 1990). In order to obtain a coherent text, 27 filler sentences were added, divided into 34 propositions (residual information). Numbers of words, syllables and characters per sentence were controlled. Appendix II shows these numbers as well as the average level of each information category in the text hierarchy (Meyer, 1975).

The 57 sentences of the text were presented one by one on a computer screen (IBM model PS/2). ${ }^{1}$ The subject pressed a button after reading the text's title on the screen. Automatically the title disappeared and the first sentence was presented in the centre of the screen. Simultaneously, in the bottom of the screen a bar was displayed, consisting of a series of red boxes. This bar represented the total time $\left(8^{\prime \prime} 30^{\prime \prime}\right)$ availlable for studying the text. As time elapsed, the red boxes turned yellow one by one. Subjects had to finish studying the complete text before the last red box had altered its color; they were not required to fully spend the total amount of available study time, however. After reading the first sentence, the subject pressed the button once more. The sentence disappeared from the screen and was immediately followed by the next one. In this fashion, all 57 sentences of the text were studied once. The procedure applied was demonstrated to the subjects with a short unrelated text. For each sentence, the time interval between the two subsequent button presses was registered as the time spent on that sentence.

The study phase was followed by a distraction task. During 20 minutes, subjects had to answer in writing a number of difficult questions about economics, for example: "What is in your opinion the net effect on the interest rate levell when both consumers spend more money and in the same time concerns invest less of their profits? Why?" (Voss, Blais, Means, Greene, \& Ahwesh, 1986). This task intended to divert the subjects from the literal wordings of the text and thus to "empty" short-term memory. Keenan and Kintsch (1974) have demonstrated that a 20-minute distraction task is sufficient to reach that goal.

The final task in this study was a free recall test. Subjects had to report orally everything they could recall of the text. Subjects' free recall was recorded on tape.

1 The diagonal computer screen size was $32 \mathrm{~cm}$; the distance between the subject and the screen was approximately $60 \mathrm{~cm}$. 


\section{RESULTS}

\section{Recall}

Verbatim transcriptions were made of the 18 recall protocols. The number of propositions correctly recalled per information category was established. Six out of the 18 recall protocols were randomly selected and scored by a second rater. The mean interrater agreement for this sample amounted $82 \%$ (ranging from 77 to $88 \%$ ). Based on this high interrater agreement, it was assumed that similar percentages of agreement would be found for the remaining 12 protocols. Therefore, these protocols were scored by only one rater.

Because of the unequal numbers of propositions and sentences per information category in the original text, the amount of recall and study time for residual information was weighed in order to permit comparisons with the three other information sets. By means of (two-way) analyses of variance and (post-hoc) Newman-Keuls comparisons the results of the three groups were analyzed; t-tests were applied to test whether a subject group differed in recall or in study time per information category.

Table 3.1 presents the mean numbers of propositions correctly recalled by the three subjects.

\section{Table 3.1}

Mean number of propositions per information category correctly recalled as a function of mobilization treatment. Local study time free and total study time fixed. The figures between brackets refer to the standard deviations.

Information category

$\begin{array}{lrrrrr}\begin{array}{l}\text { Mobilization } \\ \text { treatment }\end{array} & \text { EEC } & \text { Fish-farm } & \text { Tourism } & \text { Residual* } & \text { Totals } \\ \text { EEC group } & 12.2(4.4) & 14.5(2.4) & 3.0(1.7) & 8.5(0.8) & 38.2(8.4) \\ \text { Tourism group } & 2.1(2.4) & 3.5(2.7) & 10.9(2.2) & 4.7(2.6) & 21.1(8.6) \\ \text { Control group } & 12.9(3.0) & 9.2(2.6) & 2.4(1.2) & 7.0(1.0) & 31.5(3.4)\end{array}$

* Recall scores for residual information (34 propositions) have been transformed.

The three groups clearly showed different overall recall scores, $F(2,15)=$ $8.448, p<.01, M S_{e}=13.03$. The four categories of information were recalled differently, $F(3,45)=16.243, p<.0001, M S_{\ell}=3.57$. Furthermore, a significant 
interaction of the factors mobilization treatment and information category was found, $F(6,45)=41.961, p<.0001, M S_{\mathcal{E}}=3.57$; in other words, each group of subjects recalled the four information categories of the text in a different way. Figure 3.1 graphically displays the recall patterns, obtained in Experiment 1.

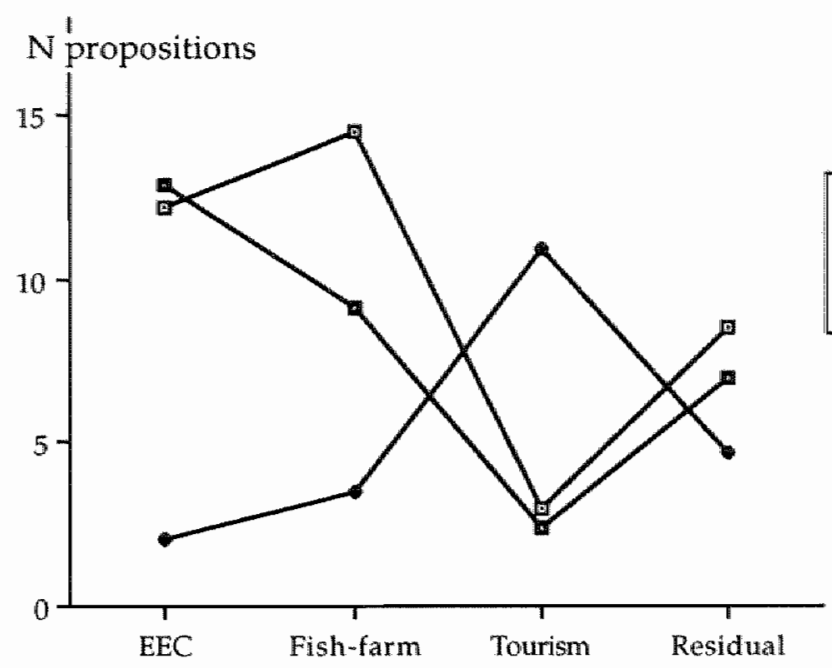

-to- EEC group

$\rightarrow$ Tourism group

$\rightarrow$ Control group

Information category

Figure 3.1 Mean number of propositions per information category correctly recalled by the three groups of subjects

Newman-Keuls comparisons (at the .05 level) demonstrated that the tourism group recalled less of the text than both the EEC group and the control group. Furthermore, the total recall score obtained by the EEC group exceeded the overall recall score of the control group but this effect did not reach the level of significance applied; the difference in total recall scores was only significant at a $10 \%$ level.

Moreover, Newman-Keuls comparisons (alpha set at .01) showed that the EEC group outperformed the control group in recall of fish-farm information. No recall differences were found between the EEC group and the control group with respect to EEC information, tourism information and residual information.

The tourism group showed a recall pattern totally different from those of the other groups, which is depicted in Figure 3.1. The tourism group obtained higher recall scores for tourism information than for EEC information $(t(5)=$ 
$8.81, p<.0001$ ), fish-farm information $(t(5)=5.201, p<.01)$ or residual information $(t(5)=2.91, p<.05)$

\section{Study time allocation}

Table 3.2 presents the mean study time spent per information category by the three subject groups, obtained in Experiment 1 in which total study time was fixed and local study time was free.

Table 3.2

Mean study time per information category, expressed in seconds, as a function of mobilization treatment. Local study time free and total study time fixed. The figures between brackets refer to the standard deviations.

Information category

Mobilization EEC Fish-farm Tourism Residual* Totals

treatment

$\begin{array}{llllll}\text { EEC group } & 93.0(17.5) & 113.7(21.1) & 65.5(7.8) & 68.6(13.5) & 340.8(53.6)\end{array}$

$\begin{array}{llllll}\text { Tourism group } \quad 54.3(8.5) & 61.2(6.9) & 109.5(10.1) & 59.2(6.9) & 284.3(22.2)\end{array}$

$\begin{array}{llllll}\text { Control group } & 117.8(10.5) & 90.1(11.3) & 64.9(9.1) & 77.7(7.5) & 350.6(25.1)\end{array}$

* Time spent on residual information ( 27 sentences) has been transformed.

None of the three groups fully utilized the total amount of available study time $\left(8^{\prime} 30^{\prime \prime}\right)$. They differed in the total amount of study time spent, $F(2,15)=$ $5.77, p<.05, M S_{e}=332.33$. Different amounts of study time were spent on the four information categories, $F(3,45)=22.351, p<.0001, M S_{e}=71.11$. The interaction of mobilization treatment and information category was significant, $F(6,45)=60.001, p<.0001, M S_{e}=71.11$; in other words, the three subject groups displayed different patterns of how the total study time was allocated to the four information sets. Figure 3.2 depicts these study time allocation patterns.

By means of post-hoc Newman-Keuls comparisons (at the .05 level), it was demonstrated that the EEC group appeared to have focused new relevant information that had not been activated previously. The EEC group processed this fish-farm information longer than the control group. On the other hand, the control group spent more processing time on EEC information, which had not been activated previously, than the EEC group. These groups 
did not differ in study time spent on tourism information and residual information.

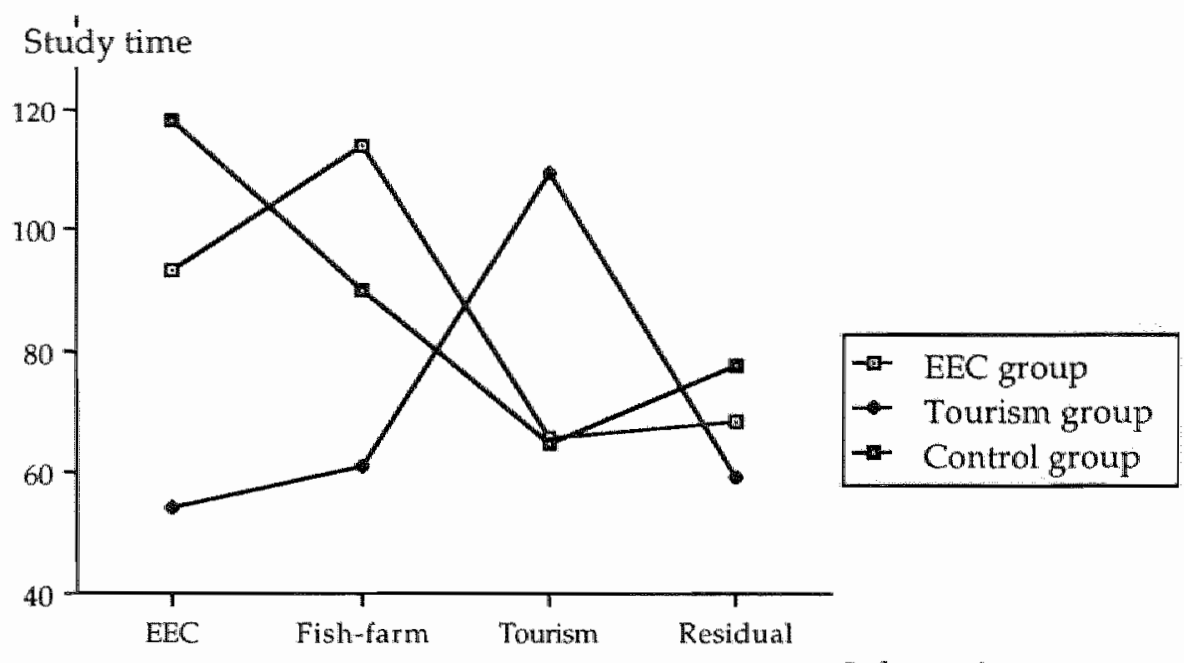

Information category

Figure 3.2 Average amount of time spent (in seconds) on each information category by the three subject groups

T-tests showed that the EEC group spent more time on relevant new fish-farm information than on EEC information $(t(5)=4.614, p<.01$ ); the EEC group also allocated more study time to fish-farm information than to tourism information $(t(5)=5.851, p<.01$ ) or residual information $(t(5)=8.265, p<.01)$. The control group, on the other hand, spent most processing time on EEC information. This information category that the control group had not activated previously was processed longer than the fish-farm information $(t(5)=4.823, p<.01)$, the tourism information $(t(5)=9.82, p<.001)$ or the residual information $(t(5)=$ $6.765, p<.01)$.

The pattern of study time allocation displayed by the tourism group completely differed from the patterns of the other subject groups. Posthoc Newman-Keuls comparisons (alpha set at .05) showed that the tourism group took in general less time to study the text than the other groups. Furthermore, the tourism group spent more time on tourism information than on EEC information $(t(5)=11.457, p<.0001)$, fish-farm 
information $(t(5)=7.453, p<.0001)$ or residual information $(t(5)=9.19$, $p<.0001)$.

\section{DISCUSSION}

The data of Experiment 1 provide some support for the cognitive set-point hypothesis. Compared to the control group, the EEC group spent less time on EEC information and more time on new relevant fish-farm information. Consequently, the EEC group outperformed the control group in recall of fishfarm information. I These data suggest that, indeed, a cognitive set-point is applied during information processing. Mobilizing prior knowledge of EEC fishing policies enabled the EEC group to process that information already prior to reading the text; hence, subjects required less time to reach the setpoint for this information and had more time available to extend their knowledge with relevant new information. An expected difference in total recall did not materialize, however. The EEC group's mean recall score of 38.2 versus the control group's score of 31.5 did not reach the required significance level. However, Newman-Keuls comparisons (at the .01 level) showed that if recall scores were corrected for amount of time spent, the EEC group clearly outperformed the control group in overall recall.

The data of Experiment 1 also support the notion that both the cognitive set-point hypothesis and the selective-attention hypothesis may explain the way in which subjects process texts, depending on the nature of the prior knowledge previously activated. It was assumed that subjects possessed a well-formed prototypical tourism schema, which would guide them through the text, discarding information that was not slot-filling. The tourism group's data support these assumptions. This group spent more time on tourism information and less on the remaining text parts. In addition, the tourism group showed better recall of this information. As expected, these subjects did not extend their knowledge base including information from other text parts. In fact, since they used only $69 \%$ of the total study time, the suggestion is not far-fetched that they were selectively searching for perspective relevant information and largely skipped all other information. These patterns of recall and study time allocation are in agreement with the selective-attention hypothesis, suggested by Anderson and his associates (Anderson \& Pichert, 1978; Goetz et al., 1983; Pichert \& Anderson, 1977). Hence, the results of Experiment 1 support the assumption put forward by Machiels-Bongaerts (1993) that both the selective-attention hypothesis and the cognitive set-point hypothesis may apply under specific conditions. If prior knowledge is orga-

1 In the General Discussion section will be dealt with the interesting finding that the control group recalled as much of the EEC information as the EEC group. 
nized in a schema, one may expect the selective-attention mechanism to be active. If, however, prior knowledge does not (yet) have a fixed cognitive structure, as was the case with the subjects' knowledge about the EEC fishery policies, one may expect subjects to behave in a way predicted by the cognitive set-point framework: They will tend to extend their knowledge base rather than just up-date it.

\section{EXPERIMENT 2}

Experiment 1 has shown that predictions by both text-processing frameworks hold under conditions when local study time is free but total study time is fixed. But what patterns of recall and study time allocation would emerge if total study time would also be free? The cognitive set-point hypothesis predicts no overall recall differences between the EEC group and the control group since all subjects would be free to reach the set-point for each information category. The EEC group, however, would require less time than the control group to reach the set-point for the EEC information and, hence, would need less time overall.

By contrast, the tourism group, will selectively attend only to schemarelevant information. Therefore, subjects are expected to spend more time on tourism information and less on all other categories; this would lead to better recall of tourism information but lower overall recall scores. In addition, the selective-attention hypothesis predicts that they would spend less time overall.

\section{SUBJECTS}

In this second experiment, 18 subjects participated, who were randomly assigned to the EEC group, the tourism group, or the control group. Subjects were undergraduate economics students at the University of Limburg. Their average age amounted to 20 years and 6 months (ranging from 19 to 29 years). For participating in this study, they received a financial compensation.

\section{PROCEDURE}

The procedure was similar to the one of Experiment 1, with one exception: Total study time was not limited; therefore, no bar of boxes, representing the available study time, appeared in the bottom of the screen on which the text was presented. 


\section{RESULTS}

\section{Recall}

Data analysis was similar to the procedure applied in Experiment 1. Again, 6 out of 18 recall protocols were randomly selected and scored by a second rater. In this second experiment, the average agreement between the two raters amounted to $81 \%$ (ranging from $70 \%$ to $92 \%$ ).

The recall patterns of the three groups of subjects, obtained in Experiment 2 in which local and, hence, total study time was free, are presented in Table 3.3.

Table 3.3

Mean number of propositions per information category correctly recalled as a function of mobilization treatment. Both local and total study time free. The figures between brackets refer to the standard deviations.

Information category

Mobilization EEC Fish-farm Tourism Residual* Totals treatment

\begin{tabular}{lrrrrr}
\hline EEC group & $14.3(1.4)$ & $14.6(1.0)$ & $5.8(2.0)$ & $11.6(0.9)$ & $46.3(4.1)$ \\
Tourism group & $1.2(1.3)$ & $1.8(1.2)$ & $14.3(1.2)$ & $7.2(2.4)$ & $24.4(4.2)$ \\
Control group & $14.6(1.2)$ & $13.8(1.9)$ & $7.1(2.3)$ & $11.8(2.4)$ & $47.3(3.8)$
\end{tabular}

* Recall scores for residual information (34 propositions) have been transformed.

Overall, the three groups of subjects differed in recall scores, $F(2,15)=60.928$, $p<.0001, M S_{e}=4.1$. Apparently, the four information categories in the text did not result in different recall scores, $F(3,45)=1.976, p=.13, M S_{e}=2.45$. However, the interaction of the factors mobilization treatment and information category was significant, $F(6,45)=78.035, p<.0001, M S_{e}=2.45$; in other words, the three groups recalled the four information categories in a different way. Figure 3.3 depicts the recall patterns of the three groups, obtained in Experiment 2.

Subsequent Newman-Keuls comparisons (alpha set at .05) demonstrated that the EEC group and the control group did not differ in total recall performances. These groups of subjects largely displayed the same recall pattern: 
No differences were found for each of the four information categories of the text.

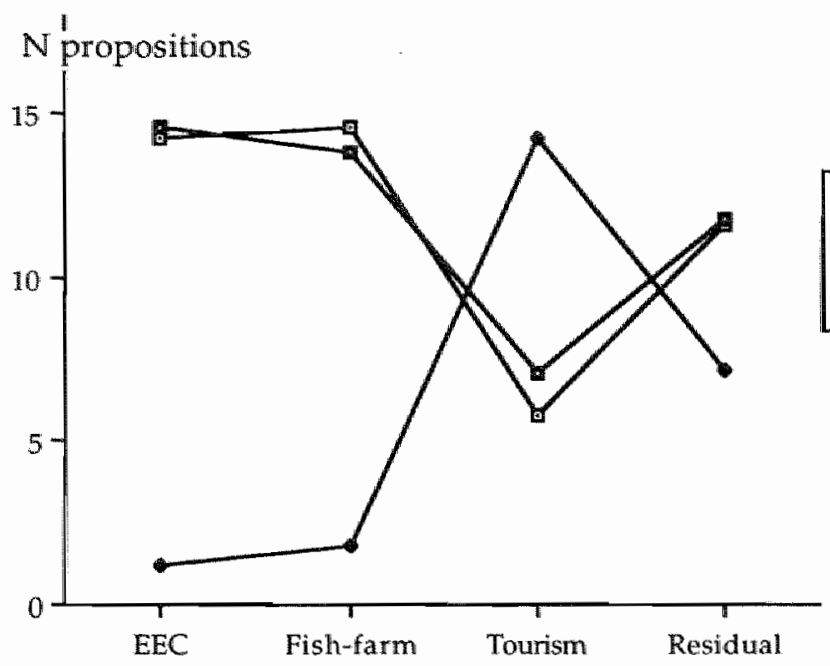

- 0 EEC group

$\rightarrow$ Tourism group

$\rightarrow$ Control group

Information category

Figure 3.3 Mean number of propositions per information category correctly recalled by the three subject groups

The tourism group was different from the other two groups in every respect. Newman-Keuls comparisons (at the .01 level) showed that subjects under this condition recalled less of the text than the EEC group or the control group. However, the tourism group recalled more of the relevant tourism information than of the EEC information $(t(5)=15.38, p<.0001)$, the fish-farm information $(t(5)=16.161, p<.0001)$ or the residual information $(t(5)=7.179, p<$ $.0001)$. The tourism group poorly recalled these categories. Except for residual information, no differences with respect to these other information categories could be found.

\section{Study time allocation}

Table 3.4 shows the mean study times spent by the three groups of subjects on each information category under the condition that both local and total study time were free.

The three groups of subjects differed in the total amount of time spent on processing the text, $F(2,15)=10.715, p<.01, M S_{e}=826.19$. The amounts of time spent on the four information sets in the text was different, $F(3,45)=$ 
29.716, $p<.0001, M S_{e}=118.82$. Furthermore, the interaction of mobilization treatment and information category was significant, $F(6,45)=82.236, p<$ $.0001, M S_{e}=118.82$; in other words, the three groups differed in the amount of study time spent per information category. Figure 3.4 graphically displays the patterns of study time allocation of the three subject groups, obtained in an entirely free processing condition.

Table 3.4

Mean study time per information category, expressed in seconds, as a function of mobilization treatment. Both local study time and total study time free. The figures between brackets refer to the standard deviations.

Information category

\begin{tabular}{lrrrrrr}
$\begin{array}{l}\text { Mobilization } \\
\text { treatment }\end{array}$ & EEC & Fish-farm & Tourism & Residual* & Totals \\
\cline { 1 - 2 } & & & & & \\
EEC group & $114.0(19.1)$ & $151.6(24.9)$ & $78.6(13.0)$ & $107.2(18.4)$ & $451.3(69.2)$ \\
Tourism group & $80.4(11.5)$ & $83.1(11.7)$ & $157.2(11.2)$ & $86.9(7.3)$ & $407.6(34.2)$ \\
Control group & $172.3(23.9)$ & $173.5(26.0)$ & $93.3(14.5)$ & $118.9(12.1)$ & $557.0(62.9)$
\end{tabular}

* Time spent on residual information (27 sentences) has been transformed.

By means of post-hoc Newman-Keuls comparisons (at the .01 level), it was demonstrated that, as was predicted by the cognitive set-point hypothesis, the control group overall required more time for text processing than the EEC group. Especially the EEC information was processed longer by the control group than by the EEC group. Both groups of subjects did not differ in study time spent on fish-farm information; neither did these groups differ in the amount of processing time spent on residual information and tourism information.

Like in Experiment 1, the tourism group displayed a pattern of study time allocation entirely different from the patterns shown by the two other groups of subjects. On processing the tourism information more study time was spent by the tourism group than either on EEC information $(t(5)=16.97$, $p<.0001)$, on fish-farm information $(t(5)=14.526, p<.0001)$ or on residual information $(t(5)=13.246, p<.0001)$. None of the further comparisons was
significant. 


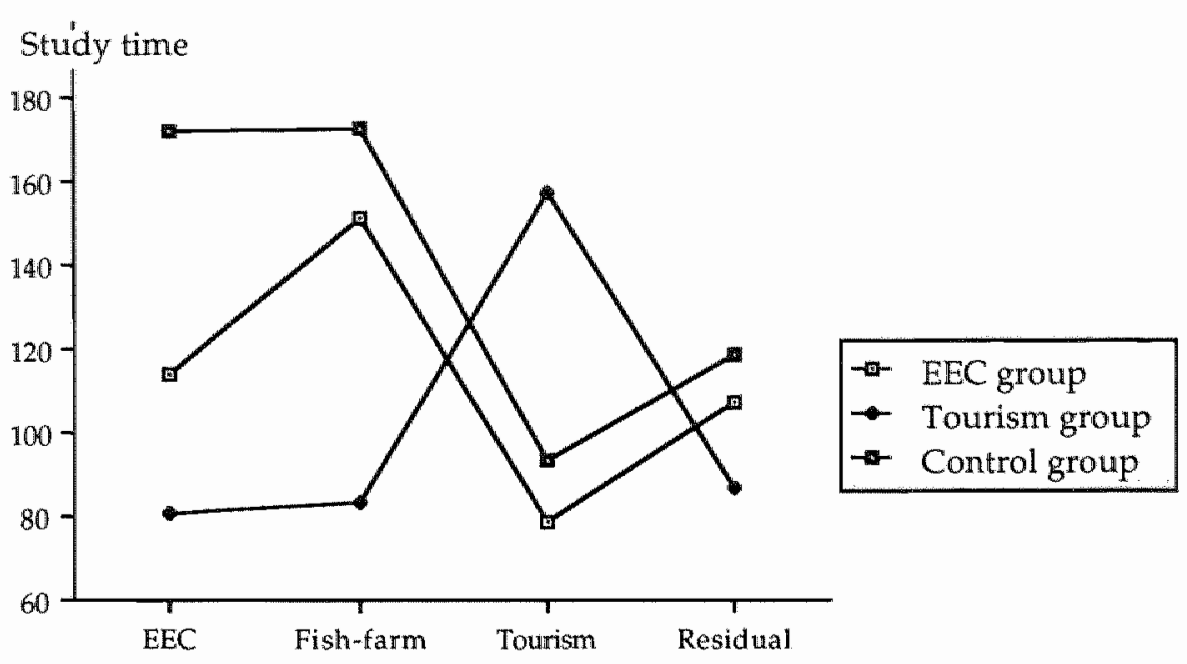

Information category

Figure 3.4 Average amount of time spent (in seconds) on each information category by the three subject groups

\section{DISCUSSION}

The results of Experiment 2, like those of Experiment 1, provide support for the predictions derived from the two hypotheses under study.

Overall, the EEC mobilization group recalled of the text as much as the control group but required less time to achieve that result. The difference in study time was located in the amount of time allocated to the EEC information. Here the EEC group spent significantly less time than the control group. These findings imply that the EEC group needed less time for that information because similar information could already be processed while mobilizing. Again, this suggests that people apply a cognitive set-point while processing a text. This cognitive set-point indicates when sufficient time has been allocated to specific information units to ensure some set levell of understanding. Since the EEC condition already processed the EEC information for some time prior to reading, subjects under this condition needed less time to reach the set-point for that information. Clearly, subjects in the mobilization condition compensate for the pre-processing event by spending less time on the relevant text paragraphs. This phenomenon was also noticed by Johnson and Kieras (1983). As predicted by the cognitive set-point hypothesis, no differences in recall materialized. It was hypothesized that under the conditions of Experiment 2, the control group would also be able to reach set-point for all 
categories of information (at the expense of more time), hence, no differences in recall would emerge.

The results of Experiment 2 illustrate once more that the tourism group processed the information in a way entirely different from the other groups. The subjects of the tourism group mainly confined themselves to selecting and processing slot-filling tourism information, resulting in increased study time and better recall.

\section{EXPERIMENT 3}

In Experiment 3 , both local and total study time were fixed. This processing condition would be a particular challenge to the selective-attention hypothesis and the cognitive set-point hypothesis since no studies are known in which predictions derived from these hypotheses were tested under this condition.

The cognitive set-point hypothesis predicts that the EEC group would only be able to benefit from her processing advantage stemming from mobilizing relevant prior knowledge if subjects were able to freely distribute study time over the various information parts of the text. In the present experiment, however, every sentence of the text was presented for a fixed amount of time. Therefore, recall differences between the EEC group and the control group were not expected to appear since the EEC group would no longer be in a position to reduce time spent on information in the text corresponding with the prior knowledge activated. Neil ther would the EEC group be able to spend more time on the relevant new fish-farm information. Thus, since all information categories would be processed equally long by the EEC group and the control $^{t}$ group, the EEC group's processing advantage would be nullified. Consequently, no recall differences were expected to arise between the EEC group and the control group under the present condition.

The selective-attention hypothesis assumes that the tourism group would mainly focus slot-filling information, as far as possible within this fixed condition, leading to better recall of that information.

\section{SUBJECTS}

In this third study, 18 subjects participated, who were all students of economy at the University of Limburg. Randomly, the subjects were assigned to the EEC group, the tourism group, or the control group. The subjects' average age was 20 years and 2 months (ranging from 18 to 24 years). For participating,
subjects received a financial compensation. 


\section{PROCEDURE}

The procedure applied only differed from the previous studies with respect to the study phase. The presentation rates of the 57 sentences of the text were fixed, based on the total available study time of Experiment $1\left(8^{\prime} 30^{\prime \prime}\right)$. Since the sentences of the EEC information, the fish-farm information and the tourism information were equal in length, the sentences were all presented for nine seconds.

\section{RESULTS}

\section{Recall}

Data analysis was similar to the procedures applied in Experiments 1 and 2. Again, 6 out of the 18 recall protocols were selected at random and scored by a second rater. In this third experiment, the average interrater agreement for this sample amounted to $85 \%$ (ranging from $75 \%$ to $91 \%$ ).

Table 3.5 presents the three groups ${ }^{\prime}$ recall scores per information catego$r y$, obtained in an entirely fixed processing condition.

Table 3.5

Mean number of propositions per information category correctly recalled as a function of mobilization treatment. Both local and total study time fixed. The figures between brackets refer to the standard deviations.

Information category

\begin{tabular}{lrrrrr}
$\begin{array}{l}\text { Mobilization } \\
\text { treatment }\end{array}$ & EEC & Fish-farm & Tourism & Residual* & Totals \\
\hline EEC group & $13.4(3.9)$ & $12.3(2.2)$ & $5.2(1.6)$ & $10.8(1.6)$ & $41.7(6.9)$ \\
Tourism group & $2.1(1.3)$ & $2.2(1.8)$ & $11.3(3.0)$ & $6.5(2.7)$ & $22.1(7.6)$ \\
Control group & $12.2(3.6)$ & $11.6(1.4)$ & $4.7(1.1)$ & $9.5(1.8)$ & $38.0(3.6)$
\end{tabular}

* Recall scores for residual information (34 propositions) have been transformed.

Overall, different numbers of propositions were correctly recalled by the three subject groups, $F(2,15)=16.532, p<.001, M S_{e}=9.83$. Furthermore, the subjects recalled unequal amounts of information of the four different categories, 
$F(3,45)=4.099, p<.05, M S_{e}=4.3$. Finally, a significant interaction of mobilization treatment and information category was found, $F(6,45)=28.558, p<$ $.0001, M S_{e}=4.3$; in other words, the three groups of subjects recalled the four information categories in a different way. Figure 3.5 presents once more the recall patterns of the three subject groups, obtained in Experiment 3 .

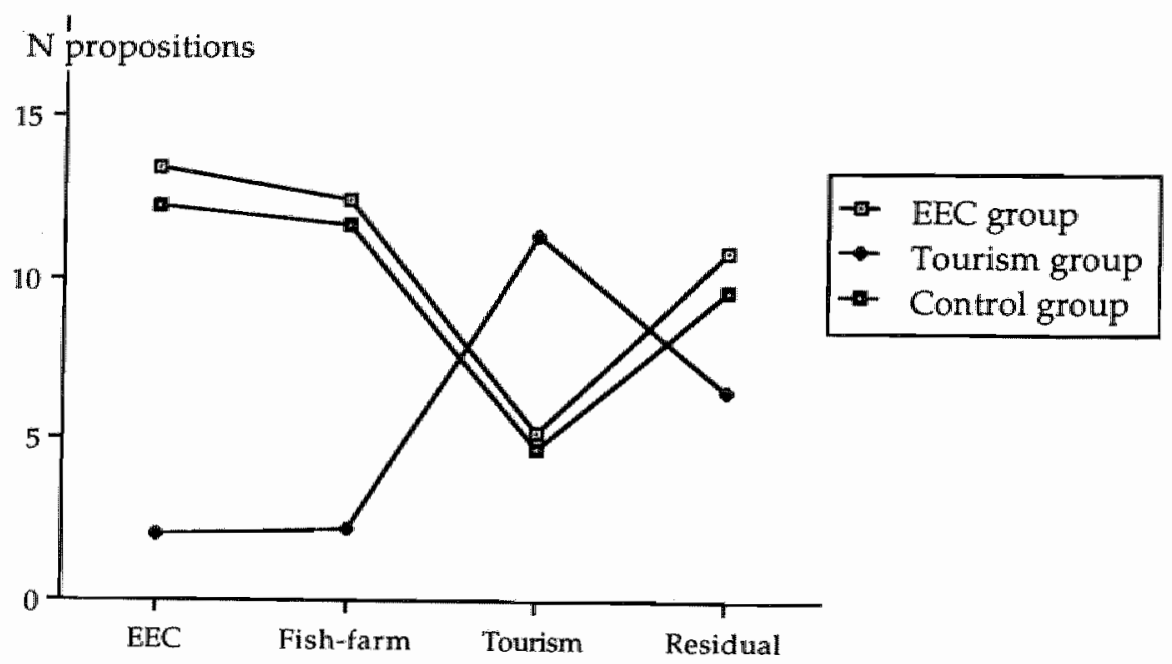

Information category

Figure 3.5 Mean number of propositions per information category correctly recalled by the three subject groups

Post-hoc Newman-Keuls comparisons (at the .05 level) showed that the EEC group and the control group did not differ in the total number of propositions correctly recalled. These two groups of subjects largelly showed identical recall patterns for each information category.

Under this third experimental manipulation of available study time, the recall pattern obtained by the tourism group again departed from those of the two other groups of subjects. Newman-Keuls comparisons (at the .01 level) demonstrated that the overall recall scores obtained by the tourism group was exceeded by the total recall scores of both the EEC group and the control group.

In this third study again, the subjects in the tourism group mainly recalled tourism information. The recall scores obtained by the tourism group for that category of information exceeded the scores for each of the other information categories: EEC information $(t(5)=8.632, p<.0001)$, fish-farm in- 
formation $(t(5)=12.324, p<.0001)$ and residual information $(t(5)=7.251, p<$ $.0001)$.

\section{DISCUSSION}

The recall scores shown by the EEC group, opposed to those of the control group, reflected the recall pattern as predicted by the cognitive set-point hypothesis; no differences in overall recall were obtained between these subject groups. The EEC group did not outperform the control group in recall of any of the four information categories in the text since this group could not benefit from the processing advantage provided by the mobilization treatment.

The performances of the tourism group confirmed the predictions of the selective-attention hypothesis. These subjects largely confined themselves to slot-filling tourism information. The remaining information categories of the text which did not match the slots of the schema previously activated were poorly recalled. In other words, although the sentences of the EEC information and the fish-farm information, both relevant information categories in the text itself, were presented for nine seconds each, low recall scores were obtained for these information sets. Consequently, the tourism group showed overall lower recall scores than the EEC group and the control group.

\section{GENERAL DISCUSSION}

The studies reported provide considerable support for the idea that humans, while processing information, use a cognitive standard for comprehension against which is judged whether sufficient processing time has been allocated in order to meet that standard. It was demonstrated that mobilization of prior knowledge, under appropriate conditions, induces subjects to spend less time on studying similar information when subsequently encountered in a text. Alternatively, subjects allocated more study time to information that extended their knowledge base beyond what was already known. In Experiment 1, under restricted processing time conditions, subjects spent $21 \%$ less time on information for which mobilization took place and $21 \%$ more time on new information that extended their knowledge base. In addition, their recall of information for which was mobilized was similar to that of a control condition, while they recalled no less than $37 \%$ more propositions of the new information. Overall recall differences between both subject groups were found to be significant only at the $10 \%$ level. The experimental group processed, however, $18 \%$ more information per unit time as a result of the mobilization treatment. In Experiment 2, both total and local study time were free. As predicted, this processing condition enabled the control group to reach set-point 
for all information categories of the text. Consequently, no differences in recall were found; neither overall, nor for each of the four categories. However, the control group needed almost $20 \%$ more study time to reach the set-point for each of the categories. As predicted by the cognitive set-point hypothesis, this difference was mainly due to the fact that the control group required significantly more time to process information for which the experimental group previously mobilized. This, again, is congruent with the idea that the processing of information is subject to a cognitive set-point which takes into account the whole processing cycle including mobilization. Experiment 3 , finally, showed that, when subjects are inhibited in freely allocating study time to different parts of a text, effects of previous processing of information disappeared. Here, processing advantages due to prior knowledge mobilization could not be translated into a different pattern of study time allocation, hence, could not lead to gains in recall by one of the subject groups.

Intriguing is the finding in Experiment 1 that the control group recalled as much EEC policy information as the mobilization group. Since this information had no special status for the control group, had not become more salient or important through any treatment, one would have expected the control subjects to recall less information of both EEC information and fishfarm information. A possible explanation might be the prominence of the EEC information in the text. In the hierarchical structure of the text, included as Appendix II, this category of information can be found at the highest level. Possibly, subjects pay special attention to hierarchy information (Meyer, 1975) and process high-level information more extensively, that is: Utilize a higher set-point for this information category. The data of Experiment 2 provide some evidence that this is indeed the case. The processing time data of the control group matched quite well with the relative importance of the different parts of the text, as indicated by the text structure in Appendix II, suggesting that the cognitive set-point for low-level information is indeed set at a lower level than for high-level information. However, Peeck and colleagues (Peeck, 1982; Peeck et al., 1982) and Schmidt and colleagues (Machiels-Bongaerts et al. in press; Schmidt, 1982b, 1983b) report identical recall patterns for different materials. Machiels-Bongaerts et al. (in press), for instance, required two groups of subjects to mobilize either names of US presidents or names of states of the Union prior to studying a list including both mobilized and nonmobilized names of each item category. As the cognitive set-point hypothesis predicts, the mobilization groups excelled in recall of non-mobilized names, but there were no differences between the experimental groups and a control condition in recall of mobilized materials. Since the list of names had no inherent structure, in the same way a text has, one is tempted to believe that this phenomenon is caused by the saliency of the material in the mind of the subjects themselves. How exactly is presently unclear. 
A second major finding of the studies described is that the cognitive set-point hypothesis and the selective-attention hypothesis are not mutually exclusive. We have demonstrated that, under specific conditions, selective attention rather than knowledge extension can be induced in subjects. In the three studies reported, the tourism group discarded the more important information about the EEC policies and their consequences in favor of parts of the text relevant to a tourism perspective but less important in the hierarchical structure of the text. We have argued that this difference between a selectiveattention strategy and a knowledge extension strategy results from the nature of the cognitive structures available to subjects. If knowledge is encapsulated in rich and highly organized schemata that have been used successfully in many relevant situations, a selective-attention approach aimed at instantiation may prevail. Such a processing strategy is highly efficient in terms of cognitive load and in terms of processing time required to comprehend a text. If, however, an appropriate schema is not available, that is: If knowledge is limited, if its structure is unstable, and if it has not yet been extensively used in a variety of situations, one may see the knowledge extension strategy in action. In the latter case, the cognitive set-point mechanism plays a crucial role in the allocation of attention and processing time, as has been documented by the studies presented.

One may argue that the phenomena described are just an expression of a reading goal induced into the reader by the particular mobilization treatment and that there is no further need for the kind of analyses reported here. The mobilization of EEC policies merely taught subjects to attend to information relevant to that perspective during information processing and the same applies to the tourism mobilization. The patterns of recall and allocation of processing time would demonstrate that the generation of the various reading goals has been successful. There is no doubt in our mind that one of the sideeffects of prior knowledge mobilization is to orient the reader with regard to the text. Otherwise, it would be difficult to understand, for instance, why the tourism group focused so much on tourism information, despite its low status in the text hierarchy. More important, however, is its effect on the way in which information is processed. What we have attempted to demonstrate, and we believe with success, is that, given a particular goal, quite different processing strategies emerge as a function of the nature of the prior knowledge available to the reader. The cognitive set-point hypothesis and the selective-attention framework exemplify these different processing strategies.

How does the idea of a cognitive set-point fit within more comprehensive theories of text processing? Recently, Kintsch (1988) has outlined a theory of text comprehension that deviates in a number of ways from the dominant schema-theoretic perspective outlined earlier. According to Kintsch, text 
comprehension can be understood as a largely bottom-up process. Knowledge is not pre-stored in fixed cognitive structures but is generated as the need arises and in the context of the particular task at hand. He assumes that knowledge can be conceived as an associative network of nodes (concepts mostly) and their interrelations. When a reader attempts to understand a text, words or phrases will activate corresponding nodes and activation will spread to other related nodes in the network. In this way, a pool of knowledge is activated that may or may not be relevant to the task of understanding the text at hand. Kintsch refers to this phase in text comprehension with the term "construction phase". While further reading, this pool of knowledge will be reduced. Processing subsequent information in the text will activate more concepts, limiting the number of plausible candidates giving meaning to the information previously encountered. In other words, the newly acquired information in the text deactivates irrelevant knowledge. During the subsequent "integration phase" of text comprehension, a coherent representation of the text is built by integrating knowledge from earlier cycles of activation with that stemming from later cycles. We assume that mobilization of relevant knowledge prior to reading a text speeds up the construction phase. As a result of mobilizing prior knowledge, some context is already provided in light of which the concepts in the text could be understood. Therefore, irrelevant knowledge may be faster deactivated and less cycles of activation may be necessary in order to create coherence. Consequently, a reader may proceed through the text at a faster rate. What we would like to add to Kintsch's ideas is the concept of a cognitive set-point as defined in this thesis. The cognitive set-point determines what level of coherence is acceptable in order to proceed to a next sentence or paragraph. This seems to be intuitivelly attractive because it clarifies that people can glance at a text to get an impression of its contents or study the same text for future information retrieval. The intention of the reader determines the set-point level and, hence, defines which degree of coherence is adequate. A strong test of these assumptions, however, requires study of text processing at a more molecular level than is possible in the present experimental paradigm. 
CHAPTER IV

\section{EFFECTS OF INSTRUCTIONAL VARIATION ON SELECTIVE ATTENTION}

\section{INTRODUCTION}

The results obtained by the tourism groups reported in Chapter III do not ultimately prove the strength of schema activation. The tourism groups seemed unable to resist the effect of schema activation on information processing and recall. Rather irrelevant information in the text itself prevailed upon far more important information. This pattern was found under all manipulations of available study time. However, these patterns of recall and study time allocation could also have emanated from a poor instruction. In order to provide the subjects a reading goal, they were told that a text contains important and less important information. It was explained to the subjects that the goal of the research they participated in was to test whether important information could be discerned from unimportant information during text processing. This introduction to the study phase would explain to the subjects why they had to press a button to be presented with the next sentence. This instruction, however, could have had an undesirable impact on the subjects in the tourism groups; they could have interpreted "important" as "important in the light of the knowledge mobilized". Thus, as a result of a misinterpreted instruction, linking prior knowledge mobilization and text processing, subjects may have assumed that they had to focus information corresponding with the knowledge previously mobilized. Consequently, if this assumption would be accurate, the patterns of recall and study time allocation reported in Chapter III may have been caused by an indigent instruction. In that case, these results 
would not support the selective-attention hypothesis but would merely confirm that the instruction applied had a not intended side-effect.

To test whether schema mobilization induces a selection process during text processing beyond merely installing a reading goal, three studies will be described. Subjects were run under two conditions: (a) Explicit instruction in which subjects were instructed to try to recall as much as possible of the complete text; and (b) implicit instruction, equaling the possibly ambiguous instruction described earlier. If the so-called explicit instruction groups would show identical patterns of study time allocation and recall as those of the tourism groups in Chapter III (the so-called implicit instruction groups) under the three manipulations of available study time, the effects found earlier cannot be attributed to a faulty instruction.

In summary: If the explicit instruction groups would obtain patterns of recall and study time similar to those of the implicit instruction groups despite the explicit instruction to try to recall as much as possible of the entire text, the impact of schema activation would be demonstrated more evidently.

\section{EXPERIMENT 1}

In Experiment 1, the total study time was fixed ( 8 minutes and 30 seconds) which could be freely allocated to the various text parts. The explicit instruction group is expected to show patterns of recall and study time largely identical to those obf the implicit instruction group in the first study of Chapter III. During text processing, tourism information would be selected and processed at length since only this information is assumed to fill slots of the schema. Consequently, tourism information would be recalled best.

\section{MATERIAL}

In the studies presented, the text "The consequences of the EEC fishery policies for Crando Douro" (see Appendix I) was applied again. For additional information with respect to the material used, see Chapter II (pp. 29 - 31).

\section{SUBJECTS}

In this study, 12 subjects participated, who were all undergraduate students of economy at the University of Limburg. Six of them formed the explicit instruction group; the implicit instruction group was identical to the tourism group of Experiment 1 described in the previous chapter. The average age of 
these subjects was 18 years and 8 months (ranging from 18 to 20 years). For participating in this experiment, they received a financial compensation.

\section{PROCEDURE}

In Experiment 1, total study time was fixed $\left(8^{\prime} 30^{\prime \prime}\right)$ which could be freely allocated to the sentences of the text. Subjects were run individually.

The study consisted of four elements: (a) Prior knowledge mobilization, (b) studying the text, (c) a diversion task, and (d) a free recall task. This procedure was similar to the one described in Chapter III (pp. 46-47), with one exception. After mobilization, subjects in the explicit instruction group were told that they had to study closely a text on a computer screen in order to recall as much as possible of the entire text. The implicit instruction group was told, as outlined before, that texts contain important and less important information. It is usually impossible to recall a text completely. Subjects in the implicit instruction group were told that the goal of the research they participated in was to investigate whether important information can be discerned from unimportant information during text processing. Consequently, study time spent on each sentence of the text would be registered. The sentences of the text had to be studied one by one. Subjects could process the text at their own pace, but they had to finish studying the complete text within the total available study time. During text processing, they could constantly check how much time was left and how many sentences they still had to study. Subjects were not required to fully use the total available study time. The procedure was demonstrated by means of a short neutral text.

\section{RESULTS}

\section{Recall}

The protocols were transcribed. The numbers of propositions recalled per information category were counted. A second rater scored 4 of the 12 protocols. The mean interrater agreement was $82 \%$ (ranging from $77 \%$ to $89 \%$ ).

The results of the two groups were analyzed by means of (two-way) analyses of variance. Newman-Keuls comparisons were applied to test whether the groups differed in the number of propositions recalled or in amount of study time spent for each information category. Finally, it was tested by means of $\mathrm{t}$-tests whether each group showed different recall scores c.q. different amounts of study time per information category. Table 4.1 presents the recall scores of the two subject groups.

The two groups differed in the total number of propositions correctly recalled $\left(F(1,10)=11.732, p<.01, M S_{\mathfrak{e}}=13.13\right)$. Subjects who were explicitly 
told to try to remember as much as possible of the text showed higher recall scores than the other subject group. The number of propositions correctly recalled differed per information category $\left(F(3,30)=39.382, p<.0001, M S_{e}=\right.$ 2.14). Furthermore, this recall pattern was not identical for the two groups of subjects, $F(3,30)=9.252, p<.001, M S_{e}=2.14$. Figure 4.1 displays the recall patterns of the two subject groups once more.

\section{Table 4.1}

Mean number of propositions per information category correctly recalled as a function of mobilization treatment. Local study time free and total study time fixed. The figures between brackets refer to the standard deviations.

Information category

\begin{tabular}{lccccc}
$\begin{array}{l}\text { Mobilization } \\
\text { treatment }\end{array}$ & EEC & Fish-farm & Tourism & Residual* & Totals \\
\hline $\begin{array}{l}\text { Implicit instruction } \\
\text { group }\end{array}$ & $2.1(2.4)$ & $3.5(2.7)$ & $10.9(2.2)$ & $4.7(2.6)$ & $21.2(8.6)$ \\
$\begin{array}{l}\text { Explicit instruction } \\
\text { group }\end{array}$ & $8.3(1.6)$ & $7.7(1.9)$ & $11.0(2.0)$ & $8.5(2.0)$ & $35.5(5.6)$ \\
\end{tabular}

* Recall scores for residual information (34 propositions) have been transformed.

Newman-Keuls comparisons (at the .01 level) showed that the explicit instruction group recalled more propositions of the EEC information, the fishfarm information and the residual information than the implicit instruction group. No recall differences were found with respect to tourism information.

The explicit instruction group, like the implicit instruction group, mainly recalled tourism information. Recall scores for tourism information exceeded the scores for EEC information $(t(5)=3.024, p<.05)$, for fish-farm information $(t(5)=3.664, p<.05)$ and for residual information $(t(5)=5.371, p<.01)$.

Furthermore, the explicit instruction group's recall pattern was compared with that of the control group described in Chapter III. This additional analysis showed that the explicit instruction group behaved in much the same way as the implicit instruction group under the present study time constraints. The only exception refers to the explicit instruction group's higher overall recall. Newman-Keuls comparisons (at the .05 level) showed that this score did not differ from the control group's total score. Still, the control group outperformed the explicit instruction group in recall of EEC information. 


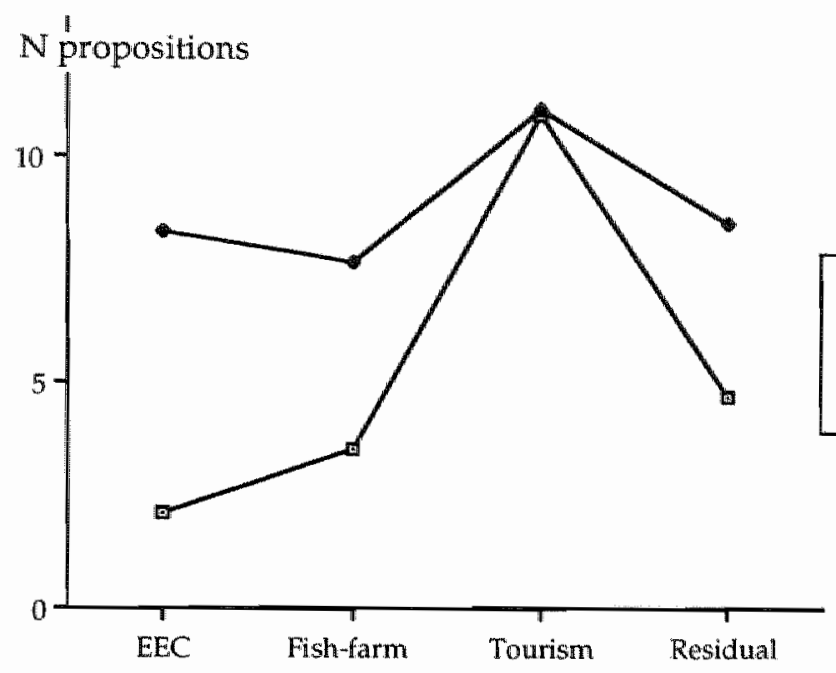

Instruction group

$\rightarrow$ Implicit

- Explicit

Information category

Figure 4.1 Mean number of propositions per information category correctly recalled by the two groups of subjects

\section{Study time allocation}

Table 4.2 presents the study time allocation patterns of the two subject groups.

Table 4.2

Mean study time per information category, expressed in seconds, as a function of mobilization treatment. Local study time free and total study time fixed. The figures between brackets refer to the standard deviations.

Information category

Mobilization EEC Fish-farm Tourism Residual* Totals
treatment

Implicit instruction $\quad 54.3(8.5) \quad 61.2(6.9) \quad 109.5(10.1) \quad 59.2(6.9) \quad 284.3(22.2)$ group

Explicit instruction $\quad 90.2(12.3) \quad 86.6(14.2) \quad 104.4(8.5) \quad 63.3(6.9) \quad 344.4(39.1)$ group

* Time spent on residual information ( 27 sentences) has been transformed. 
The two groups differed in the total amount of time spent on text processing $\left(F(1,10)=10.739, p<.05, M S_{\mathscr{E}}=252.7\right)$. The total available study time was not fully utilized by each group. Different amounts of study time were spent on the four information categories, $\left(F(3,30)=117.789, p<.0001, M S_{e}=39.6\right)$. Furthermore, the interaction of the nature of the instruction and information category was significant $\left(F(3,30)=27.01, p<.0001, M S_{e}=39.6\right)$. Thus, the two groups displayed different patterns of how the total available study time was allocated to the four information categories, which is shown in Figure 4.2.

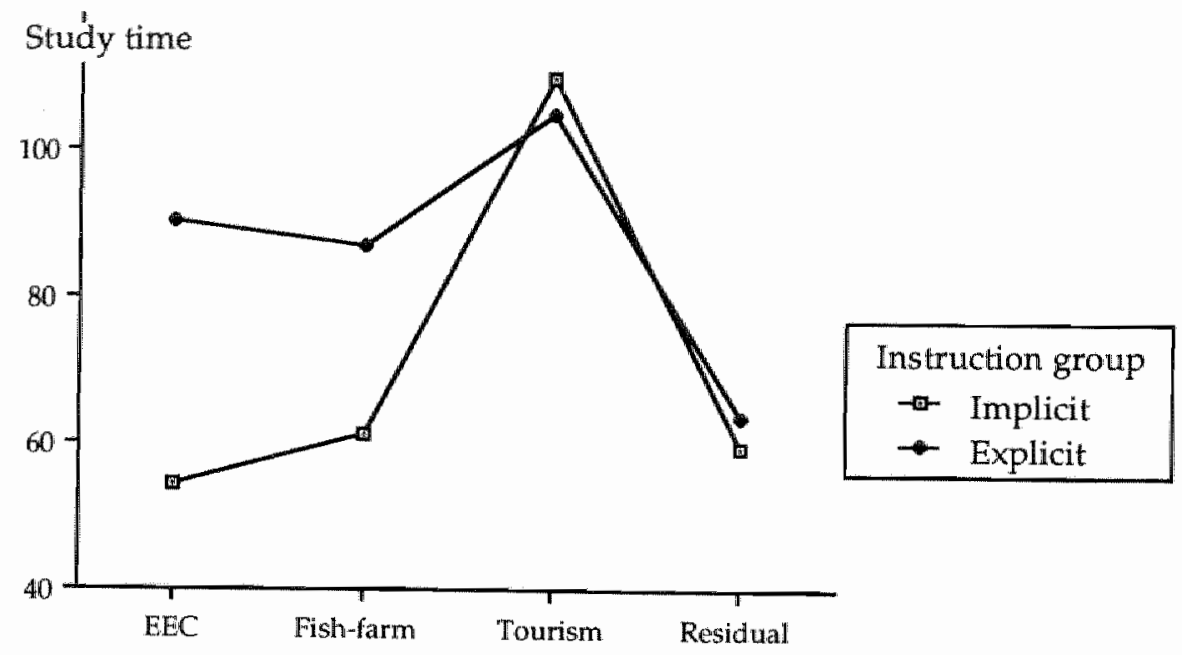

Information category

Figure 4.2 Average amount of time spent (in seconds) on each information category by the two subject groups

Newman-Keuls comparison (alpha set at .01) showed that the explicit instruction group spent more time on EEC information and fish-farm information than the implicit instruction group. The two groups did not differ in amounts of study time spent on both residual information and tourism information.

Yet, the explicit instruction group spent most study time on tourism information. This information category was studied longer than the EEC information $(t(5)=4.256, p<.01)$, the fish-farm information $(t(5)=4.024, p<.05)$ and the residual information $(t(5)=17.804, p<.0001)$.

Furthermore, the explicit instruction group was compared to the control group, whose processing data were reported in the first study of Chapter III. Although the way of information processing of the explicit instruction group 
appeared to have been influenced by the modified instruction, the pattern of study time allocation was to a large extent as deviant from the control group's pattern as the pattern shown by the implicit instruction group. The main difference between the explicit instruction group and the implicit instruction group was that the latter group in total used less time to study the text than the control group, whereas the explicit instruction group did not $(F(2,15)<1)$. Yet, although the explicit instruction group, compared to the implicit instruction group, showed an increase in time spent on EEC information and fishfarm information, Newman-Keuls comparisons (at the .05 level) revealed that these information categories were processed longer by the control group.

\section{DISCUSSION}

To some extent, the explicit instruction group appeared to have "utilized" the available amount of study time $\left(8^{\prime} 30^{\prime \prime}\right)$ better than the implicit instruction group. More time was spent on information categories placed on high levels in the text's hierarchical structure. This pattern of study time allocation resulted in overall higher recall. However, most study time was still spent on processing tourism information. Consequently, this category of information was recalled best. These patterns of recall and study time allocation largely resemble those of the implicit instruction group, both providing support for the selective-attention hypothesis. In conclusion, despite the explicit instruction to recall as much as possible of the text as a whole, the explicit instruction group, like the implicit instruction group, primarily recalled slot-filling information, showing the strong impact of schema activation.

EXPERIMENT 2

In this study, total and local study time were free. Under these processing conditions, the explicit instruction group was assumed to reflect the predictions of the selective-attention hypothesis again. Most time would be spent on selecting slot-filling tourism information, resulting in higher recall of this information. Thus, the explicit instruction group was expected to show largely the same patterns of study time and recall as the implicit instruction group.

\section{SUBJECTS}

In this study, 12 subjects participated. Six of them formed the explicit instruction group; the implicit instruction group was the tourism group described in Experiment 2 of the previous chapter. The subjects' average age was 19 years 
and 7 months (ranging from 19 to 29 years). Subjects, who were all undergraduate students economics at the University of Limburg, received a financial compensation for participating in this study.

\section{PROCEDURE}

The procedure was identical to the procedure applied in Experiment 1, except for the study phase. No time bar was presented in the bottom of the computer screen since the total amount of available study time was free.

\section{RESULTS}

\section{Recall}

For each subject, the number of propositions correctly recalled per information category was established. Like in the previous study, 4 of the 12 protocols were selected at random and scored by a second rater. In this study, the average interrater agreement a mounted to $86 \%$ (ranging from $75 \%$ to $90 \%$ ).

Table 4.3 shows the number of propositions correctly recalled per information category by the two groups when total and local study time were free.

Table 4.3

Mean number of propositions per information category correctly recalled as a function of mobilization treatment. Both local and total study time free. The figures between brackets refer to the standard deviations.

Information category

Mobilization EEC Fish-farm Tourism Residual* Totals
treatment

Implicit instruction group

Explicit instruction group
$1.2(1.3)$

$1.8(1.2)$

$14.2(1.2)$

$7.2(2.4)$

$24.4(8.6)$

$8.8(1.7)$

$9.0(2.4)$

$11.3(3.8)$

$7.8(1.7)$

$37.0(3.4)$

* Recall scores for residual information (34 propositions) have been transformed.

The implicit instruction group and the explicit instruction group differed in overall recall scores, $F(1,10)=15.313, p<.01, M S_{\mathcal{E}}=7.76$. Different recall 
scores were obtained for the four information categories in the text, $F(3,30)=$ $43.62, p<.0001, M S_{e}=3.53$. Furthermore, the interaction of the nature of instruction and item category was significant, $F(3,30)=22.539, p<.0001, M S_{e}=$ 3.53 ; in other words, the two groups did not recall the four information categories in the same way. Figure 4.3 depicts these recall patterns of the two groups, obtained in Experiment 2.

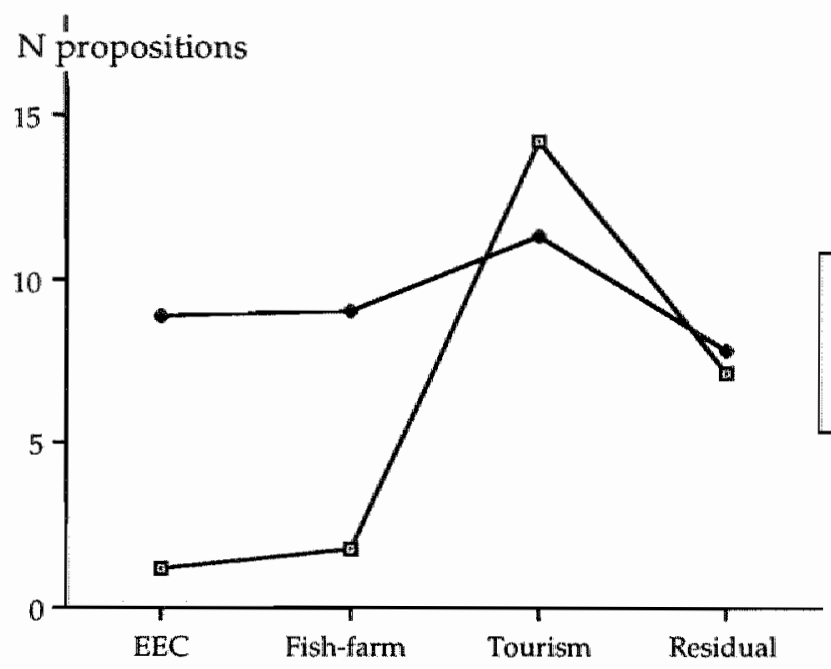

Instruction group

$\rightarrow$ Implicit

- Explicit

Information category

Figure 4.3 Mean number of propositions per information category correctly recalled by the two groups of subjects

Newman-Keuls comparisons (alpha set at .01) showed that the explicit instruction group outperformed the implicit instruction group in recall of EEC information and fish-farm information. No recall differences were found between the two groups for tourism information and residual information.

As Figure 4.3 shows, the explicit instruction group"s recall pattern did not appear to be as capricious as that of the implicit instruction group. Like the implicit instruction group, the explicit instruction group still recalled the slotfilling information best. However, as t-tests showed, recall of tourism information no longer prevailed; this information was not recalled better than the EEC information $(t(5)=1.648, p=.16)$ or the fish-farm information $(t(5)=$ 1.257, $p=.26$ ). The explicit instruction group's recall of tourism information only (marginally) exceeded the recall of residual information $(t(5)=2.291, p=$ $.07)$. 
Also in this second study, the recall pattern of the explicit instruction group was compared to the control group's recall pattern, described in the second study of Chapter III. Newman-Keuls comparisons (at the .01 level) showed that the explicit instruction group was, like the implicit instruction group, outperformed by the control group in recall of EEC information, fishfarm information and residual information; like the implicit instruction group, the explicit instruction group obtained higher recall scores for tourism information than the control group. In conclusion: The explicit instruction group appeared to differ from the control group in largely the same way as the implicit instruction group in an entirely free processing condition.

\section{Study time allocation}

Table 4.4 presents the mean study time spent on each information category of the text by the two groups when both local and total study time were free.

Table 4.4

Mean study time per information category, expressed in seconds, as a function of mobilization treatment. Local and total study time free. The figures between brackets refer to the standard deviations.

Information category

Mobilization EEC Fish-farm Tourism Residual* Totals

treatment

Implicit instruction $\quad 80.4(11.5) \quad 83.1(11.7) \quad 157.2(11.3) \quad 86.9(7.3) \quad 407.6(34.2)$ group

Explicit instruction $\quad 112.9(39.1) \quad 105.6(35.4) \quad 135.2(42.8) \quad 73.4(24.5) \quad 427.1(118.3)$ group:

* Time spent on residual information ( 27 sentences) has been transformed.

The two subject groups did not differ in the total amount of time used for studying the text $(F(1,10)<1)$. Different amounts of study time were spent on the four information categories $\left(F(3,30)=160.563, p<.0001, M S_{e}=62.3\right)$. A significant interaction between nature of instruction and information category was found $\left(F(3,30)=34.241, p<.0001, M S_{e}=62.3\right)$; in other words, the two groups differed in the way in which study time was distributed over the four information categories of the text. Figure 4.4 graphically presents these stuidy time allocation patterns of the two groups. 


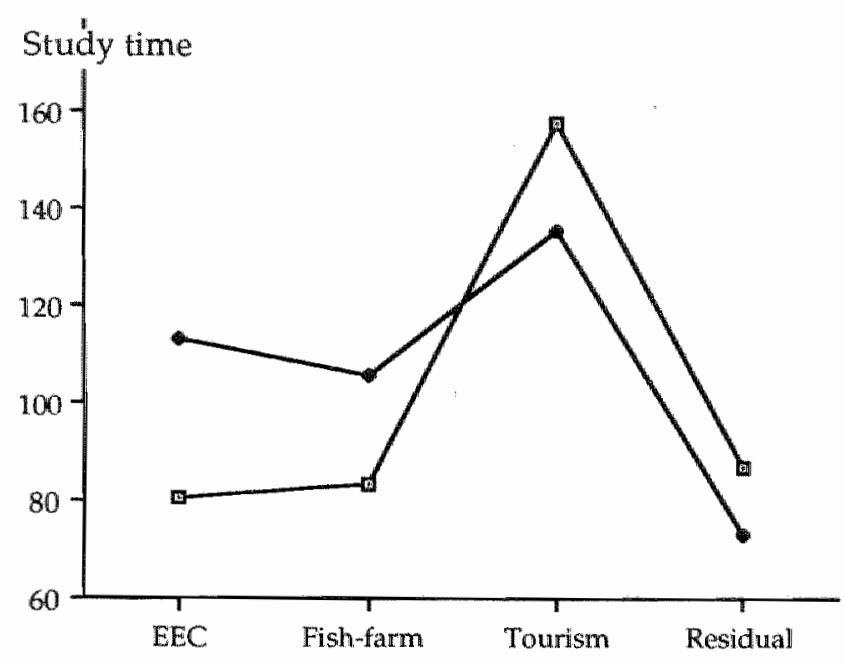

Instruction group

$\rightarrow$ Implicit

- Explicit

Information category

Figure 4.4 Average amount of time spent (in seconds) on each information category by the two subject groups

Newman-Keuls comparisons (alpha set at .05) showed that the explicit instruction group spent more time on EEC information than the implicit instruction group. The two groups did not differ in time spent on fish-farm information, tourism information and residual information.

The explicit instruction group, like the implicit instruction group, spent most processing time on tourism information. The explicit instruction group spent more study time on this information category than on EEC information $(t(5)=12.666, p<.0001)$, fish-farm information $(t(5)=8.483, p<.001)$ or residual information $(t(5)=8.122, p<.001)$. However, the study time allocation patterns of the two groups were not fully identical. The explicit instruction group spent more study time on the important information categories in the text itself; the EEC information and the fish-farm information, than on residual information $(t(5)=6.526, p<.01$ and $t(5)=6.314, p<.01$, respectively), whereas the implicit instruction group did not show these differences.

The study time allocation pattern of the explicit instruction group was compared to that of the control group, reported in Experiment 2 of the pre vious chapter. Newman-Keuls comparisons (at the .01 level) showed that the explicit instruction group differed from the control group in largely the same way as the implicit instruction group. The explicit instruction group spent more time on processing tourism information than the control group, whereas the opposite held for the remaining information categories of the text. 


\section{DISCUSSION}

From the results obtained in Experiment 2, it can be concluded once more that the explicit instruction group was influenced by the changed instruction to some extent. The explicit instruction group showed overall higher recall scores, which could be attributed to better recall of EEC information and fishfarm information. Yet, the impact of schema activation could not be overruled. Even for the explicit instruction group, recall of slot-filling tourism information was superior to all remaining information categories. Furthermore, the patterns of recall and study time allocation obtained by the explicit instruction group were largely as deviant from those of the control group as the implicit instruction group's patterns. This demonstrates that despite the explicit instruction to try to remember as much as possible from the entire text, the strong impact of schema mobilization on information processing and subsequent recall could not be overruled by the more explicit instruction.

\section{EXPERIMENT 3}

In this study, total and local study time were fixed. Under these processing constraints, the explicit instruction group is expected to show recall patterns similar to those of the implicit instruction group (i.e., the tourism group described in Experiment 3 of the previous chapter). Despite the explicit instruction to try to recall as much as possible of the text was a whole, it is hypothesized that the explicit instruction group would not be able to overrule the impact of schema activation. Consequently, this group would excel in recall of tourism information. This recall pattern would confirm the predictions of the selective-attention hypothesis under a more stringent instruction condition.

\section{SUBJECTS}

In this study, 12 subjects participated, all undergraduate students economics at the University of Limburg. Six of them formed the explicit instruction group; the implicit instruction group was identical with the tourism group of the third study in Chapter III. Subjects' average age was 19 years and 8 months (ranging from 19 to 21 years). Subjects were financially compensated.

\section{PROCEDURE}

The procedure was similar to those applied in Experiments 1 and 2, except for the study phase. Both total and local study time were fixed in this study. 


\section{RESULTS}

\section{Recall}

The recall protocols were transcribed. The number of propositions correctly recalled per information category was established. Again, 4 out of the 12 recall protocols were randomly selected and scored by a second rater. The mean interrater agreement was $89 \%$ (ranging from $82 \%$ to $96 \%$ ).

Table 4.5 presents the number of propositions correctly recalled per information category by the two groups of subjects, obtained in Experiment 3.

\section{Table 4.5}

Mean number of propositions per information category correctly recalled as a function of mobilization treatment. Both local and total study time fixed. The figures between brackets refer to the standard deviations.

\section{Information category}

Mobilization $\quad$ EEC Fish-farm Tourism Residual* Totals
treatment

Implicit instruction group

Explicit instruction group
$2.1(1.3)$

$2.2(1.8)$

$11.3(3.0)$

$6.5(2.7)$

$22.1(7.6)$

$5.4(1.4)$

$11.2(3.1)$

$29.5(5.0)$

${ }^{\star}$ Recall scores for residual information (34 propositions) have been transformed.

The two groups did not differ in overall recall scores, $F(1,10)=4.013, p=.07$, $M S e=10.28$. Different numbers of propositions were recalled of the four information categories, $F(3,30)=60.195, p<.0001, M S e=2.41$. Furthermore, this pattern was not identical for each group; in other words, the interaction of the nature of instruction and information category was significant, $F(3,30)=$ $3.979, p<.05, M S e=2.41$. Figure 4.5 displays the recall patterns of the two subject groups, obtained in an entirely fixed processing condition.

Post-hoc Newman-Keuls comparisons (at the .01 level) showed that the explicit instruction group outperformed the implicit instruction group in recall of both EEC information and fish-farm information. The two groups did not differ in recall of tourism information and residual information.

Like the implicit instruction group, the explicit instruction group mainly recalled slot-filling tourism information. This score exceeded the recall scores 
obtained for EEC information $(t(5)=7.556, p<.001)$, fish-farm information $(t(5)=3.78, p<.05)$ and residual information $(t(5)=3.615, p<.05)$. Furthermore, the explicit instruction group recalled the EEC information better than the residual information $(t(5)=4.824, p<.01)$.

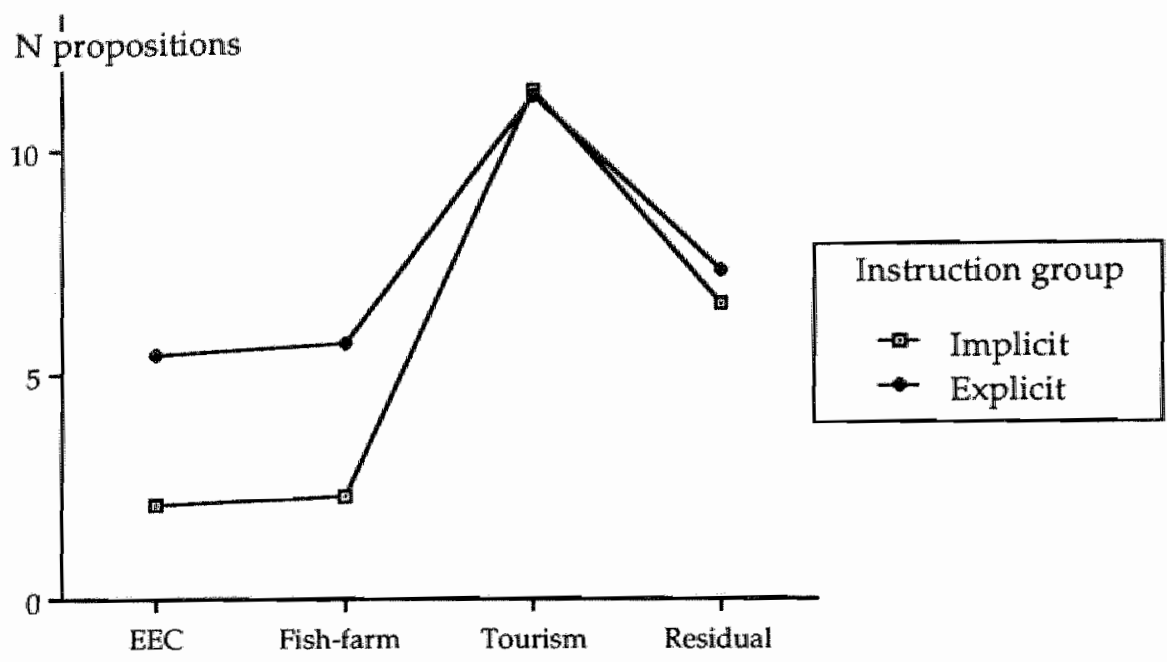

Information category

Figure 4.5 Mean number of propositions per information category correctly recalled by the two groups of subjects

It was tested whether the recall pattern of the explicit instruction group differed from that of the control group, described in the final study of Chapter III, in the same way as the implicit instruction group's pattern. In this respect, the explicit instruction group did not differ from the implicit instruction group. Newman-Keuls comparisons (at the .01 level) showed that the explicit instruction group, like the implicit instruction group, was outperformed in recall of EEC information, fish-farm information and residual information by the control group. The explicit instruction group and the implicit instruction group, on the other hand, outperformed the control group in recall of tourism information.

\section{DISCUSSION}

Also in this entirely fixed condition, the two groups appeared to have applied themselves to recall of tourism information. The implicit instruction group 
was outperformed by the explicit instruction group in recall of the most important information categories in the text, the EEC information and the fishfarm information, which can be attributed to the modified instruction. Yet, recall of tourism information, that is assumed to correspond with slots of the schema previously activated appeared to be predominant in this entirely fixed processing condition. Thus, also the explicit instruction group recalled less of information that had a high rank in the text hierarchy than of lower rank tourism information. Even though the sentences of the important EEC information and fish-farm information were presented for a fixed amount of time, these categories were still recalled worse than slot-filling tourism information.

In summary: Despite the explicit instruction to try to recall as much as possible of the text as a whole, the explicit instruction group largely retained to the way of information processing as observed in the previous studies: Focusing slot-filling tourism information. Consequently, higher recall scores were obtained for this information than for any of the remaining information categories. Despite the modified instruction and the fixed presentation rate of high rank EEC information and fish-farm information, this information was poorly recalled since it did not fill slots of the schema previously activated.

\section{GENERAL DISCUSSION}

The studies presented were designed to investigate the hypothesis that the impact of schema mobilization cannot be nullified by a different instruction. It was hypothesized that the strong effects stemming from schema activation, as observed in the studies reported in Chapter III, could be attributed to an unintended interpretation of the instruction applied. The tourism groups could have interpreted "important information" as "relevant to the schema previously activated". To resolve this problem, subjects were instructed in the present experiments to try to remember as much as possible of the entire text.

Apparently, the explicit instruction group was influenced by this modified instruction to some extent. This group spent more time on EEC information and fish-farm information than the implicit instruction group; consequently, this group was outperformed by the explicit instruction group in recall of these information categories. Yet, the explicit instruction group seemed to have been unable to override the effects of schema mobilization since this group, like the implicit instruction group, spent most time on tourism information. This pattern of study time allocation resulted in higher recall scores for tourism information than for any of the remaining information categories. The only exception to this rule was found in Experiment 2. In this entirely free condition, the explicit instruction group seemed to have obtained higher recall scores for tourism information than for the other information categories (see Figure 4.3); yet, none of these differences appeared to be significant. 
Furthermore, both the patterns of recall and study time allocation displayed by the explicit instruction group were different from those of the control group, reported in the previous chapter, to about the same extent as the patterns of the implicit instruction group. The explicit instruction group appeared to have tried to carry out the explicit instruction to try to recall as much as possible of the complete text to a somewhat larger extent than the implicit instruction group since the former group required more time to process the text when both total and local study time were free (Experiment 2). Moreover, the explicit instruction group spent more time on EEC information and fish-farm information than the implicit instruction group, but the control group spent even more time on these information categories; consequently, the control group outperformed the explicit instruction group in recall of EEC information and fish-farm information, both important in the text itself.

In summary: The impact of schema mobilization seemed to be inescapable. Despite the explicit instruction to recall as much as possible of the text as a whole, slot-filling tourism information was processed longer than the remaining information categories. This pattern of study time allocation was found, despite the fact that the latter categories of information were more important in the structure of the text itself. This way of information processing (mainly) resulted in overall higher recall scores for tourism information.

The explicit instruction group's patterns of recall and study time are in line with the findings of Schraw and Wade (1991). These researchers divided Pichert and Anderson's (1977) text, describing the adventures of two boys playing hooky from school, into two information categories: (a) Information relevant to the perspective from which the text ought to be read, and (b) information important in the hierarchical text structure. Again, perspective assignment resulted in schema activation. Consequently, perspective relevant information was recalled best, confirming the predictions of the selectiveattention hypothesis. Of the remaining information, structural important information was recalled better than structural unimportant information, which is expected if a specific reading goal is lacking (Albrecht \& O'Brien, 1991). Schraw and Wade concluded that subjects seem to apply rather complex ways of information processing. First, they select and process information relevant to the perspective assigned; subsequently, they resort to structural important information. Lee-Sammons and Whitney (1991) report similar findings.

Overall, it can be concluded that schema mobilization instigates a process of selective attention during text processing. Most time is paid to information corresponding with the schema resulting in better recall of that information. The explicit instruction group largely replicated the implicit instruction group's patterns of recall and study time. Therefore, it can be concluded that the results in Chapter III support the selective-attention hypothesis and do not confirm that the tourism groups' instruction had an unintended side-effect. 
CHAPTER V

\section{EFFECTS OF EXTENDING RELEVANT INFORMATION IN TEXT ON SELECTIVE ATTENTION}

\section{INTRODUCTION}

While interpreting the results of the tourism groups which were presented in three studies in the preceding chapter, two difficulties were encountered.

First, induced by prior knowledge activation, tourism subjects might erroneously have assumed that during text processing tourism information had to be focused, ignoring the remaining information. To check whether the patterns of recall and study time could indeed be attributed to the impact of schema activation and not to an unintended side-effect of the instruction, a new set of studies was conducted, which were presented in Chapter IV. Subjects were explicitly instructed to try to remember as much as possible of the text as a whole. Despite this modified instruction, information corresponding with the knowledge previously activated was still recalled best. This was the more interesting since that information was rather unimportant in the text itself. From these findings, it was concluded that instruction could not overrule the effects of schema activation on subsequent text processing.

A second difficulty encountered was that the tourism group did not seem to benefit from prior knowledge activation to the same extent as the EEC group. Since the EEC group and the tourism group activated relevant prior knowledge before text processing, they were both assumed to outperform the control group in overall recall. However, the EEC group and even the control group obtained overall higher recall scores than the tourism group. It was hypothesized that these recall differences may have been due to the way in 
which the text was constructed. The original text was divided into three parts: EEC information, fish-farm information and tourism information, each comprising ten sentences or 22 propositions. One could argue, however, that the EEC and the fish-farm information actually formed one text part. Although the EEC group activated prior knowledge corresponding only with the $\mathrm{EEC}$ information, the fish-farm information could be considered as a logical extension of this information. Consequently, the text would have contained more propositions relevant to the EEC group (44 propositions) than to the tourism group ( 22 propositions). The tourism group's overall lower recall may be stemming from this difference in relevant information in the text.

To test this assumption, the original text was rewritten. The number of propositions relevant to the tourism group was extended, equaling the number available for the EEC subjects under the assumption that the EEC and the fish-farm information were interpreted as one category. Thus, the new text (Appendix III) contained: (a) EEC and fish-farm information, the most important information in the text, each consisting of ten sentences or 22 propositions; and (b) tourism information, rather unimportant in the text hierarchy, consisting of 20 sentences or 44 propositions. To build a coherent text, 21 filler sentences were added; this residual information consisted of 27 propositions. ${ }^{1}$ Henceforth, the term "non-tourism information" will be applied to refer to EEC plus fish-farm information (20 sentences, 44 propositions). This non-tourism information was assumed to be relevant for the EEC group, whereas the tourism information would be relevant for the tourism group.

The new text was applied in a free processing condition. The tourism group was expected to reflect the predictions of the selective-attention hypothesis: Selectively focusing tourism information, corresponding with the knowledge activated. Non-tourism information would be ignored since it would not fill slots in the schema. The EEC group was expected to show recall and study time patterns as predicted by the set-point hypothesis. Compared to the control group, the EEC group would require less time to reach the set-point for non-tourism information since a part of this information, the EEC information, was already processed to some extent at mobilization. Consequently, the control group would require overall more time than the EEC group to obtain similar overall recall scores. These results would be identical to those found in the second study of Chapter III. However, if the assumption is correct that the tourism group's lower recall in previous studies was not so much the result of a particular processing strategy, based on a particular knowledge structure, but simply stemmed from the fact that there was less to recall for that group, recall levels similar to those of the EEC group would be expected. Thus, the tourism group was expected to benefit from prior knowledge activation to the same extent as the EEC group, which,

1 Appendix IV shows the relative importance level of each information category. 
under the present processing conditions, would result in overall lower study times than the control group to achieve overall similar recall scores.

In summary: Under the assumption that not a difference in processing strategy, but simply the number of relevant propositions could be held responsible for the tourism groups' findings, the EEC group and the tourism group were expected to benefit from prior knowledge activation largely to the same extent. Overall, the control group would require more time to obtain total recall scores, equaling the EEC group's and the tourism group's scores. Naturally, the latter groups were still expected to show different processing strategies, resulting in different recall patterns due to activating prior knowledge that differed in nature. The EEC group is expected to spend most time on non-tourism information and the tourism group would focus tourism information. Consequently, the EEC group would excel in recall of non-tourism information and the tourism group in recall of tourism information. Total numbers of propositions recalled, however, would be the same for all groups.

EXPERIMENT

\section{SUBJECTS}

In this study, 18 subjects participated, all undergraduate students economics at the University of Limburg. They were randomly assigned to the EEC, the tourism, or the control group. Their mean age was 20 years and 2 months (ranging from 18 to 26 years). Subjects received a financial compensation.

\section{PROCEDURE}

The procedure was identical to that of Experiment 2 of Chapter III. Prior to the study phase, in which local and total study time were free, subjects were explicitly instructed to try to recall as much as possible of the text as a whole.

\section{RESULTS}

\section{Recall}

The 18 recall protocols were transcribed. For each recall protocol, the number of propositions correctly recalled per information category was established. Six out of 18 protocols were randomly selected and scored by a second rater. The mean interrater agreement was $89 \%$ (ranging from $87 \%$ to $92 \%$ ).

Two-way analyses of variance were applied to analyze the three groups' results. Newman-Keuls comparisons were applied to test whether the groups 
differed in recall scores or in amount of study time spent per information category. T-tests were used to test whether within groups differences were found in recall or study time spent per information category.

Table 5.1 shows the recall scores obtained by the three subject groups.

\section{Table 5.1}

Mean number of propositions per information category correctly recalled as a function of mobilization treatment. Both local and total study time free. The figures between brackets refer to the standard deviations.

Information category

\begin{tabular}{lccc}
$\begin{array}{l}\text { Mobilization } \\
\text { treatment }\end{array}$ & Tourism & Non-tourism & Totals \\
\hline EEC group & $17.8(4.7)$ & $33.4(3.4)$ & $51.2(3.8)$ \\
Tourism group & $26.4(2.7)$ & $8.5(2.4)$ & $34.9(3.9)$ \\
Control group & $15.8(1.9)$ & $32.6(4.0)$ & $48.4(6.0)$
\end{tabular}

The three groups differed in overall recall, $F(2,15)=22.488, p<.001, M S_{\mathcal{U}}=$ 10.09. The two information sets were not recalled equally well, $F(1,15)=$ $17.525, p<.001, M S_{e}=11.99$. Furthermore, the interaction of treatment and information category was significant, $F(2,15)=97.139, p<.0001, M S_{e}=$ 11.99; thus, the three groups differed in the way in which these information categories were recalled. Figure 5.1 presents these recall patterns.

Newman-Keuls comparisons (at the .01 level) showed that the tourism group was outperformed in total recall by the EEC group and the control group, whereas the latter two groups did not differ in this respect. The same pattern was found for non-tourism information: The EEC group and the control group did not differ in recall of non-tourism information, whereas these groups outperformed the tourism group. The reversed pattern was found for tourism information: The tourism group outperformed the EEC group and control group, whereas these groups did not differ in this respect. 1

The EEC group and the control group excelled in recall of non-tourism information. Their recall scores for this information exceeded the scores for tourism information $(t(5)=5.283, p<.01$ and $t(5)=14.263, p<.0001$, respec-

\footnotetext{
${ }^{1}$ Newman-Keuls comparisons showed that the tourism group (10.4) was also outperformed by the EEC group (14.1) and the control group (13.8) in recall of residual information.
} 
tively). The tourism group, on the other hand, obtained higher recall scores for tourism than for non-tourism information $(t(5)=13.268, p<.0001)$.
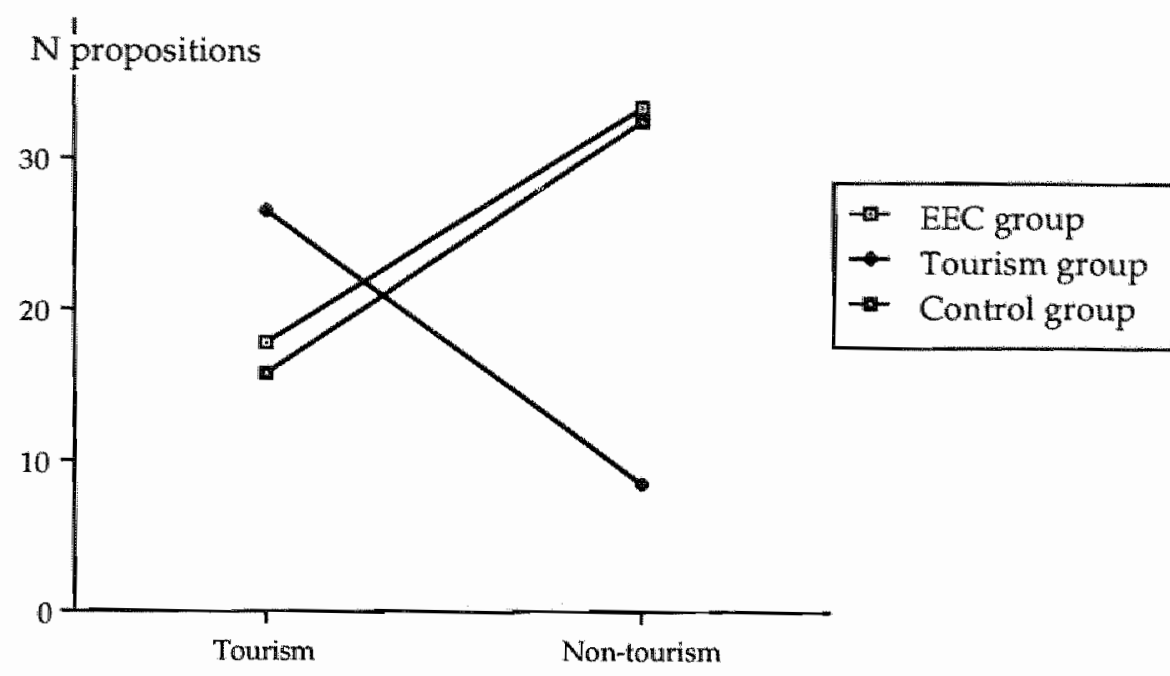

Information category

Figure 5.1 Mean number of propositions per information category correctly recalled by the three groups of subjects

\section{Study time allocation}

Table 5.2 presents the patterns of study time allocation of the three groups.

The three groups differed in total time spent, $F(2,15)=12.564, p<.001$, $M S_{e}=556.04$. The information sets were not studied equally long, $F(1,15)=$ $366.866, p<.0001, M S_{e}=321.26$. The interaction of treatment and information category was significant, $F(2,15)=62.274, p<.0001, M S_{e}=321.26$. Figure 5.2 on page 87 shows these patterns of study time allocation.

Newman-Keuls comparisons (at the 01 level) revealed that the control group spent more time on the two information categories than the EEC group or the tourism group; these groups did not differ in this respect. Moreover, the tourism group spent more time on tourism information than the EEC group and the control group, whereas the latter groups studied the non-tourism information longer than the tourism group. Furthermore, the control group spent more time on non-tourism information than the EEC group. ${ }^{1}$

1 To complete the report of all findings: The EEC group, the tourism group and the control group did not differ in amount of study time spent on residual information (108.4, 105.0 and 117.2 seconds, respectively). 
Table 5.2

Mean study time per information category, expressed in seconds, as a function of mobilization treatment. Both local and total study time free. The figures between brackets refer to the standard deviations.

Information category

\begin{tabular}{lccc}
$\begin{array}{l}\text { Mobilization } \\
\text { treatment }\end{array}$ & Tourism & Non-tourism & Totals \\
\hline EEC group & $97.3(17.0)$ & $277.6(19.5)$ & $375.0(31.4)$ \\
Tourism group & $151.7(8.8)$ & $241.6(10.8)$ & $393.3(16.5)$ \\
Control group & $106.6(14.9)$ & $359.6(39.3)$ & $466.2(45.6)$
\end{tabular}

Remarkably, the tourism group spent more time on non-tourism information than on tourism information $(t(5)=20.119, p<.0001)$. Both the EEC group and the control group showed the same pattern of study time allocation: More study time was spent on-tourism information than on tourism information $(t(5)=23.3, p<.0001$ and $t(5)=16.279, p<.0001$, respectively).

\section{GENERAL DISCUSSION}

A difficulty encountered while interpreting the findings obtained in Chapter III was that the tourism group did not seem to benefit from prior knowledge activation to the same extent as the EEC group. Like the EEC group, the tourism group was assumed to have a lead over the control group as a result of activating relevant prior knowledge. Yet, the control group outperformed the tourism group in overall recall. We assumed that selective attention was responsible for this finding. However, the tourism group's overall lower recall may also have been due to the way in which the original text was constructed. Under the assumption that the EEC group had interpreted the EEC and the fish-farm information as one information category, the original text would have contained twice as much information relevant to the EEC group than to the tourism group. Therefore, the initial text was rewritten. The number of propositions relevant to the tourism group was extended, equaling the number of propositions relevant to the EEC group. Assumed that the findings reported in Chapter III were not due to differences in processing strategy, but to the lower number of relevant propositions available, the tourism group was 
expected to benefit from prior knowledge activation to the same extent as the EEC group in the study presented. In the present processing condition, the tourism group would be able to obtain overall recall scores similar to those of the EEC group and the control group. The control group would, however, require substantially more time to achieve this score. Although the EEC group and the tourism group would not differ in overall recall, they were still expected to show different recall patterns as a result of activating knowledge that differed in nature, reflecting the predictions of the cognitive set-point hypothesis and the selective-attention hypothesis respectively. The EEC group was expected to obtain higher scores for non-tourism information than for tourism information, whereas the opposite would hold for the tourism group.

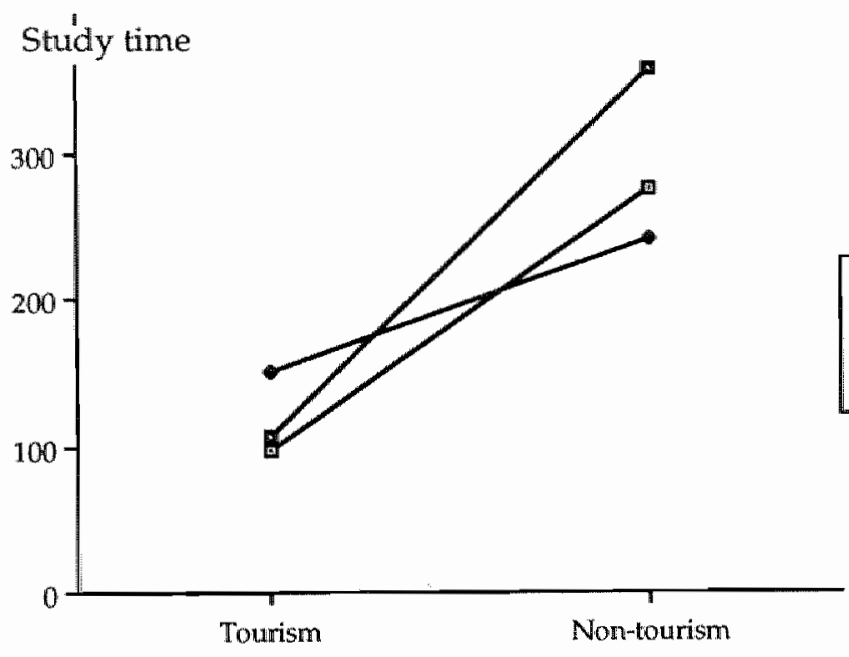

- EEC group

- Tourism group

- Control group

Information category

Figure 5.2 Average amount of study time spent (in seconds) per information category by each subject group

However, despite the relatively increased recall of tourism information, the tourism group still showed lower overall recall; this suggests that the EEC group and the tourism group indeed applied different processing strategies. Apparently, the facilitation effects emerging from activating knowledge represented in an associative net (Kintsch, 1988) differ from those stemming from schema activation (Goetz et al., 1983; Pichert \& Anderson, 1977; Vonk, 1986).

The structure of the latter type of knowledge representation may account for the tourism group's overall lower recall. A schema is a rather fixed cogni- 
tive structure, containing slots which can take a number of variables within a restricted range. Doubling the amount of slot-filling information does not automatically have to lead to a huge rise in recall. If the slot "cultural activities" is already filled with "going to bull-fights", "visiting places of pilgrimage", and "admiring old crafts like pottery and needle-point works", there would be no need (or perhaps, no space) to incorporate "folk-dances" too. The first three values would suffice to conclude that the location is very traditional, offering a variety of cultural activities. A closer scrutiny of the text applied showed that the extended tourism information provided multiple values for one slot. This may explain why subjects just recalled a few values for each slot, resulting in overall lower recall than the EEC group. ${ }^{1}$

Thus, the incorporation of slot-filling information into a ready-made knowledge structure seems to be restricted, whereas the extension of unstable knowledge, represented in a loosely interrelated network seems to be in principle unlimited. Naturally, this only holds as long as the associative net does not evolve into a ready-made cognitive structure through compilation (Anderson, 1983, 1987) or encapsulation (Schmidt \& Boshuizen, 1992).

It seems that there remains one alternative explanation for the difference in overall recall of the EEC group and the tourism group. The levels effect (Britton et al., 1979; Hustinx, 1985) may perhaps also account for the findings obtained. Meyer (1975) showed that information lower in the text hierarchy is more poorly recalled than high-level information since subjects tend to concentrate on main ideas in a text. Since tourism information was situated fairly low in the hierarchy, one would normally expect this information to be poorly recalled. But this poor recall is always at the expense of better recall of high-level information. Here is where the tourism group shows atypical behavior. Despite the fact that a fair amount of time has been spent on highlevel non-tourism information (in fact the tourism group spent more time on that category than the control group), recall remains poor. It seems that the tourism group has made an attempt to incorporate that information in the text's representation, but failed. The tourism schema active did not allow for inclusion of non-tourism information. However, a final test of our hypotheses concerning differences between schema and non-schema based processing would require textual material in which the possibility of a levels effect is excluded. We will not attempt such experiments in the context of this thesis. It is clear, however, that conducting such studies is a matter of some urgency if one wants to straighten out the possible effect of text hierarchy.

1 This would also explain why the tourism group spent relatively little time on tourism information. After selecting and processing a number of values filling a particular slot, the remaining propositions matching this slot could largely be skipped. This information process would reduce the mean time spent on tourism information. 
CHAPTER VI

\title{
MOBILIZING CATEGORY NAMES: STUDIES ON FREE RECALL AND STUDY TIME ALLOCATION1
}

\begin{abstract}
In this study, three experiments examine the effects of mobilizing prior knowledge on information processing. Subjects generated names of either US presidents or states before processing a relevant list. Study time allocated to different parts of the list and free recall were recorded. In Experiment 1, study time was unlimited; no recall differences were found but experimental subjects spent less time studying the material. In Experiment 2, total study time was fixed, but subjects were free to allocate the available time to individual items. Experimental subjects spent less time on items in the mobilized category and recalled more items. This facilitative effect of mobilization extended or "spilled over" from mobilized to non-mobilized information. In Experiment 3 , where both time for studying individual items and, hence, total study time were fixed, experimental subjects recalled more mobilized category items than controls. These results were interpreted in terms of a cognitive set-point regulating the amount of time spent on different parts of the material.
\end{abstract}

1 This chapter will be published as: Machiels-Bongaerts, M., Schmidt, H..G., and Boshuizen, H.P.A. (1993). Effects of mobilizing prior knowledge on information processing: Studies of free recall and allocation of study time. British foumal of Psychology, 4, 481-498. The experiments described were funded by grant \#7000 from the Institule for Educational Research in the Netherlands (SVO). The authors wish to acknowledge Herco Fonteijn and Eugène Custers for their comments on earlier versions of this chapter. 
Mobilization reduces the time required to reach criterion set for mobilized category items during study since these items have been processed previously. Finally, these studies prove that the set-point hypothesis applies not only to items actually mobilized but also to non-mobilized same-category items, probably through a process of spreading activation at mobilization.

\section{INTRODUCTION}

Which names would come to Dutch students' minds if they were asked to list as many US presidents as possible? They would probably mention Bush, Reagan, Kennedy, Nixon and a few other well-known presidents. If they were subsequently shown a list of US presidents' names in random order (Bush, Hayes, Coolidge, Nixon, Polk, Tyler, Reagan) and asked to recall the items on this list after a 20-minute delay, one might expect that they would recall their initial list better than the subsequent list. The basis for this expectation would be the so-called generation effect (Hirshman \& Bjork, 1988; Nairne \& Widner, 1987). This effect refers to the phenomenon that self-generated items are recalled more easily than items presented by an experimenter. According to Slamecka and Graf (1978), the generation of items would "serve (...) to increase their subsequent memorability" (p. 603).

Although this recall pattern seems plausible, a quite different pattern was found by a number of researchers. Peeck (1982) presented two experimental groups and one control group with a list of 16 names of states of the Union and 16 US presidents and asked them to study these items. Prior to the study phase, one experimental group had been asked to bring to mind (to mobilize) as many names as possible of US presidents and the other had to mobilize states of the Union. Control subjects were asked to generate names of birds and other animals. Subsequently, all subjects were shown slides with names of both presidents and states in one of three random orders. They were instructed to study each item and try to recall as many as possible. After a delay of 15 minutes, subjects had to write down all items they recalled. Experimental groups performed better than controls, an effect that could be attributed to higher recall of the mobilized category as opposed to the nonmobilized category. Thus, subjects who mobilized names of presidents recalled more presidents than the states group or the control group, whereas the states group recalled more state names than the other two groups.

However, this recall pattern could not be attributed to the generation effect. Peeck (1982) compared recall frequencies for items that were most frequently mobilized and items infrequently mobilized. If the generation effect provides a plausible explanation for the recall pattern, the experimental subjects should show higher recall than controls with respect to names frequently mobilized. No differences would be expected with respect to infrequently 
mobilized items. However, the experimental groups performed better than the control group for frequently mobilized items as well as for same-category items infrequently mobilized. This analysis shows that the generation effect cannot provide a plausible explanation for the results obtained.

Peeck et al. (1982) and Schmidt (1982b) found similar recall patterns using texts rather than lists. Subjects were presented with a text containing information that either matched or did not match previously mobilized knowledge. In both studies, the experimental group's recall exceeded the control group's, which, surprisingly, could largely be attributed to better recall of non-mobilized information, as if the effect of mobilization "spilled over" to non-mobilized information. In summary, contrary to the predictions of the generation hypothesis, prior knowledge mobilization seems to facilitate not only recall of mobilized information but also of non-mobilized information.

Schmidt and his colleagues (Machiels-Bongaerts et al., 1990; Schmidt, 1982a, 1983b) have proposed a cognitive set-point hypothesis to explain this phenomenon. According to this hypothesis, during the processing of information the cognitive system employs a set-point which indicates when sufficient time has been spent on processing that information in order to remember it. When the set-point for a certain information unit is reached, the processing system proceeds to the next unit, and so forth. This theory provides an explanation for the obvious observation that readers do not go on incessantly processing the same information. The set-point level determines the processing limit and is determined by a number of factors, such as the complexity of the study material, the reader's goal and level of fatigue.

However, during mobilization certain items have already been activated and processed. When studying a list, such items will require less processing time and therefore more time will be available for items not previously mobilized. Thus, this theory predicts an overall facilitation effect of mobilization extending to non-mobillized information when total study time is fixed, but can be freely allocated to any part of the information as was the case in the Peeck et al. (1982) and Schmidt (1982b) studies. However, the set-point hypothesis also predicts that, when total study time is unlimited both experimental and control subjects will reach the set-point for all items, and therefore show similar recall scores. Since a control group does not mobilize relevant prior knowledge in advance and, hence, cannot establish a processing advantage similar to the experimental subjects, it follows that control subjects can be expected to require more time for studying the material. In addition, one would expect experimental subjects to spend less time on mobilized items as compared to non-mobilized items, simply because some time had already been spent on that material prior to studying the target material.

However, preliminary studies (Machiels-Bongaerts \& Boshuizen, 1989) suggest that the set-point hypothesis may not only explain study time and re- 
call patterns of items mobilized but also seems to apply to non-mobilized items in the same category. Their data suggest that during mobilization of information spreading activation seems to occur to items that are not retrieved but are nevertheless processed to some extent. For instance, the nonmobilized item "Johnson" could receive spreading activation from the mobilized item "Kennedy". If spreading activation occurs during the mobilization of items of a certain category, one would expect non-mobilized items of the same category to have the same results as mobilized items in terms of study time and recall. In the present experiments, this assumption was tested by comparing allocation of study time and recall patterns of mobilized, samecategory non-mobilized and non-category items. If same-category nonmobilized items receive spreading activation during mobilization (and if the set-point hypothesis applies to them as well), it can be predicted that the cognitive set-point for these items will be reached quicker than for noncategory items requiring shorter study times and achieving better recall.

In summary: According to the set-point hypothesis, mobilizing relevant knowledge prior to a processing event has two effects, both resulting in a processing advantage. First, it influences the way in which subjects allocate available study time which, in turn, affects recall. Second, non-mobilized items receive spreading activation during mobilization. Consequently, when the list is presented these items will be processed like mobilized items as opposed to non-category non-mobilized items.

In order to test these predictions of the set-point hypothesis, three studies were conducted. The study material was a list of 32 items, 16 names of US presidents and 16 names of states of the Union adapted from Peeck (1982). Prior knowledge was mobilized by encouraging subjects to bring to mind as many names as possible of US presidents (the presidents group) or states of the Union (the states group). A control group generated as many names of composers as possible. In these studies, total time available to process the 32item list (the so-called total study time) was manipulated as well as the time available to study each list item individually (the so-called local study time). Total and local study time were either free (at subjects' discretion) or fixed (study time was limited by the experimenter). These manipulations result in three possible combinations of study time: (a) Total and (hence) local study time free, (b) total study time fixed by local study time free, and (c) both fixed.

In Experiment 1, both local and total study time were free; subjects were free to decide how much time they would spend on processing each item. The cognitive set-point hypothesis predicts that processing information relevant to prior knowledge mobilized requires less time than processing nonmobilized information. Thus, it was expected that the presidents group would pay less time to president names than to state names, whereas the states group was expected to show the reverse. The control group was ex- 
pected to allocate an equal amount of time to president names and state names since neither of these categories had been generated during mobilization. In addition, the control group would spend more time studying the list than either the presidents or the states group, since it would need more time to reach set-point for the items studied. When available study time is free, the cognitive set-point hypothesis predicts that no differences in recall will occur between the experimental groups and the control group, since each group will compensate by spending more time studying items for which no prior activation took place. In addition, it was expected that experimental subjects would allocate less time to non-mobilized same-category items as a result of spreading activation taking place while mobilizing prior knowledge.

In Experiment 2, total study time was limited, but the subjects were free to allocate this amount of time to items in the two categories since local study time was free. It was expected that the experimental groups would spend relatively less time on mobilized information, since these items had already been processed to a certain degree during mobilization, and relatively more time on non-mobilized information. According to the cognitive set-point hypothesis, this processing pattern would result in two recall phenomena. First, overall recall of the experimental groups would exceed recall of the control group owing to their processing advantage. Second, since each of the experimental groups had relatively more time available to process non-category items, superior recall of the experimental groups would be expected for both mobilized and non-mobilized categories, accounting for the "spilling-over" effect observed in other studies (Peeck et al., 1982; Schmidt, 1982b). Finally, effects of spreading activation during mobilization would be similar to those in Experiment 1 and would result in shorter processing times for nonmobilized same-category items than for non-mobilized non-category items.

In Experiment 3, both total study time and local study time were fixed. So, subjects were presented with each item for a fixed amount of time. According to the cognitive set-point hypothesis, under this fixed study-time condition, the processing advantage acquired through mobilization would lead to superior recall of the mobilized category by the experimental groups and, hence, better overall recall. This effect would be strengthened by the spreading activation received by non-mobilized same-category items.

EXPERIMENT 1

\section{SUBJECTS}

Subjects were 18 students of the University of Limburg, randomly assigned to three conditions that differed in the experimental task to be carried out during the mobilization stage. 


\section{PROCEDURE}

Subjects were tested individually. The procedure consisted of four stages: (a) Mobilization, (b) studying the list, (c) a distraction interval, and (d) a free recall test. During mobilization subjects were asked to generate as many names as possible of US presidents, of states of the Union or as a control condition, of composers. Names mobilized were recorded on audiotape. In the study stage subjects were presented with the names of 16 US presidents and 16 states. Each item appeared separately on a computer screen (IBM model PS /2; diagonal screen size 13 in). The subject's distance from the screen was approximately 25 in.

Half of the list was assumed to be well known to all subjects; the other half of the list consisted of names that were considered to be less familiar or even entirely unfamiliar. This classification was based upon the results of a pilot study (Machiels-Bongaerts \& Boshuizen, 1989). In that pillot study, two subject groups either mobilized names of US presidents or states of the Union. Items that were often mobilized and items with a low mobilization frequency were selected for the present studies.

All subjects were presented with a list of US presidents and states of the Union in one of three random presentation orders and informed of this procedure. They were instructed to study each item and try to remember as many items as possible. They were also requested to try to remember the item's category. President names were underlined. Subjects could decide for themselves how much time they would spend on each item. By pushing a button the first list item appeared. This item disappeared from the screen when the button was pushed again and the next item on the list was presented automatically. Study time for each item was measured. Subjects could oniy see each item once. After the study stage, each subject had to write the answer to a number of unrelated questions. This distraction task took 20 minutes. Finally, subjects were asked to recall every item they could remember as well as the item's category. Subjects' free recall was recorded on audiotape.

\section{ANALYSIS}

For the experimental groups the number of correctly mobilized names of either US presidents or states of the Union was counted. The number of matches between mobilized items and list items was also established. For each subject, the number of correctly recalled items was calculated as well as the mean study times in seconds for the two item categories. 


\section{RESULTS AND DISCUSSION}

Mobilization protocols of the two experimental groups showed a difference in the number of correctly mobilized items $(F(1,10)=8.767, p<.05)$. The mean number of correctly mobilized names was 7.833 for the presidents group and 10.5 for the states group. The number of mobilized items matching the 32 items on the list, however, was similar for the two subject groups $(F(1,10)<$ 1). The presidents group had previously mobilized $29 \%$ of the list items. The match between mobilized items and list items was $32 \%$ for the states group. The mobilization data in Experiments 2 and 3 were similar to the data reported in Experiment 1.

Table 6.1 shows the mean study time of the three groups for the two categories.

Table 6.1

Mean study time per item, expressed in seconds, as a function of mobilization treatment. Both local and total study time free. The figures between brackets refer to the standard deviations.

Item category

\begin{tabular}{llll}
$\begin{array}{l}\text { Mobilization } \\
\text { treatment }\end{array}$ & Presidents & States & Means \\
\hline Presidents group & $4.93(1.45)$ & $8.94(2.86)$ & 6.93 \\
States group & $8.58(2.65)$ & $4.81(1.18)$ & 6.70 \\
Control group & $9.57(2.63)$ & $9.41(2.56)$ & 9.49
\end{tabular}

The three subject groups differed in the amount of total study time required to study the 32 items in the list at the $10 \%$ level $(F(2,15)=3.181, p=.07)$. Equal amounts of study time were spent on the two categories $(F(1,15)<1)$. A significant interaction of treatment and item category was found $(F(2,15)=$ $45.181, p<.0001)$. The expected pattern of study time allocation was found for processing both president and state names, as was shown by means of subsequent Newman-Keuls comparisons (at the .05 level). The presidents group required less study time for president names than the control group or the states group. The states group needed less time for studying state names than the control group or the presidents group. Furthermore, as t-tests re- 
vealed, the presidents group spent less study time on processing president names than on state names $(t(5)=5.42, p<.01)$. The reverse pattern was obtained for the states group $(t(5)=6.162, p<.01)$, whereas the control group did not differ in this respect $(t(5)=1.149, p=30)$. To test the assumption that spreading activation occurred during mobilization, facilitating recall of nonmobilized same-category items, an additional analysis was conducted. For this analysis the 32 list items were subdivided into three item classes: (a) Mobilized items, (b) non-mobilized same-category items, and (c) items of the other non-mobilized category. If spreading activation had occurred during mobilization, the processing of non-mobilized items within the category activated would require less processing time than items of the non-activated category, since their set-points would be reached quicker. Of course mobilized items would require the smallest amount of study time to reach the cognitive set-point. Table 6.2 shows the results of this analysis.

\section{Table 6.2}

Mean study time per item, expressed in seconds, as a function of mobilization treatment. Both local and total study time free. The figures between brackets refer to the standard deviations.

Item category

Mobilization $\quad$ Mobilized Non-mobilized Non-category
treatment

Presidents group

$3.27(0.90)$

$6.59(2.09)$

$8.94(2.86)$

States group

$3.22(0.93)$

$6.40(1.80)$

8.58 (2.65)

The presidents group and the states group did not differ in total amount of study time spent $(F(1,10)<1)$. Unequal amounts of study time were spent on the three item categories $(F(2,20)=61.885, p<.0001)$. The two experimental groups showed the same pattern of study time allocation to the three item types $(F(2,20)<1)$. As predicted, a linear effect was found $(F(1,10)=$ $122.447, p<.001)$. Mobilized items required less time than non-mobilized same-category items. On the other hand, items in the non-mobilized category were processed longer than non-mobilized same-category items. Thus, these results support the assumption that spreading activation occurs during mobilization, reducing the amount of study time required to reach the set-point for non-mobilized items within the category activated. 
Table 6.3 presents the mean numbers of names of presidents and states correctly recalled by the three groups of subjects, obtained in an entirely free processing condition.

Table 6.3

Mean number of correctly recalled items as a function of mobillization treatment. Both local and total study time free. The figures between brackets refer to the standard deviations.

Item category

\begin{tabular}{|c|c|c|}
\hline Mobilization & Presidents & States \\
\hline
\end{tabular}

Presidents group

$10.17(0.98)$

$9.83(0.75)$

10.00

States group

$10.17(0.98)$

$10.17(1.47)$

10.17

Control group

The three subject groups did not differ in mean number of items correctly recalled $(F(2,15)=1.159, p=.34)$. The two item categories did not differ in level of difficulty $(F(1,15)<1)$. The interaction between treatment and category was not significant $(F(2,15)<1)$. As was demonstrated by means of Newman-Keuls comparisons, no recall differences between the three subject groups were found for either president or state names.

These results confirm the predictions of the set-point hypothesis. As a result of mobilizing relevant prior knowledge the two experimental groups gained a processing advantage over the control group. Processing information previously mobilized required less time than studying non-mobilized information, since the set-point for these items was reached quicker. Thus, the presidents group needed less study time than the control group to reach the cognitive set-point for the president names, whereas the states group needed less time than the control group to reach the set-point for the state names. As predicted, the control group required more time to study the list. It was assumed that this would happen because the control group would need more time to reach the set-point for the various items. As a result, the control group showed similar recall to the two experimental groups. Since the subjects were entirely free to spend as much time they thought they needed to 
study the items, it is difficult to see how any cognitive mechanism besides a set-point for information processing could account for these results.

Furthermore, the study time data indicate that spreading activation from mobilized to non-mobillized items must have occurred during mobilization. These data confirm the prediction of the cognitive set-point hypothesis that mobilizing prior knowledge facilitates the processing of both mobilized and non-mobilized items of the item category previously activated.

EXPERIMENT 2

In Experiment 2 total study time was fixed, but since local study time was free, the subjects could freely allocate the available time to items in either of the two categories. The cognitive set-point hypothesis predicts that the presidents group and the states group would allot less processing time to mobilized items since these items had already been processed to a certain degree during mobilization. Furthermore, it was expected that the processing of non-mobilized items within the category activated was facilitated by spreading activation during mobilization, although to a lesser extent than mobilized items. Therefore, the experimental groups could allocate relatively more time to items of the non-mobilized category. In addition to this timeallocation pattern, it was predicted that under this condition recall of the experimental groups would exceed that of the control group. Thus, the facilitation effect of mobilizing prior knowledge was expected to extend from mobilized information to non-mobilized information.

\section{SUBJECTS}

Subjects were 18 students of the University of Limburg, with six randomly assigned to each of the three conditions.

\section{PROCEDURE}

Basically the same procedure was used as in Experiment 1. Experiment 2 only differed from the previous study with respect to the study stage. In Experiment 2, total study time was fixed, but could be freely allocated to the 32 items. The maximum amount of available study time in this condition was two minutes and 15 seconds. Within this fixed amount of time, subjects had to finish studying the item list, but were not obliged to use the whole of this total time. While studying the items presented, subjects could observe how much time was left from the permitted study time. When the first item 
was presented in the center of the screen, a row of 27 red boxes appeared at the bottom of the screen. This row represented the amount of total study time. After five seconds, the first red box turned yellow; five seconds later, the next box turned yellow and so on. Subjects had to finish studying the list before all boxes in the row had changed color. A new item was presented every time the subject pushed the button. This way, all items on the list appeared once.

\section{RESULTS AND DISCUSSION}

Table 6.4 shows the two groups' mean study times for the two categories.

\section{Table 6.4}

Mean study time per item, expressed in seconds, as a function of mobilization treatment. Local study time free and total study time fixed. The figures between brackets refer to the standard deviations.

\section{Item category}

\begin{tabular}{llll}
$\begin{array}{l}\text { Mobilization } \\
\text { treatment }\end{array}$ & Presidents & States & Means \\
\hline Presidents group & $2.89(0.08)$ & $4.51(0.37)$ & 3.70 \\
States group & $4.52(0.17)$ & $2.99(0.28)$ & 3.76 \\
Control group & $4.01(0.26)$ & $3.92(0.21)$ & 3.96
\end{tabular}

Not surprisingly, the three groups did not differ in the amount of total time spent $(F(2,15)=2.591, p=.11)$ since total study time was fixed in this study. Equal amounts of time were spent on the two categories $(F(1,15)<1)$. The interaction of treatment and category was significant $(F(2,15)=241.064, p<$ .0001 ). Newman-Keuls comparisons (at the .01 level) showed that the presidents group spent less time on president names than both the states group and the control group. The state group spent less time on state names than the presidents group or the control group. Furthermore, the presidents group paid more attention to state names than to president names $(t(5)=10.832, p<$ $.0001)$, whereas the states group spent more time on president names than on state names $(t(5)=19.254, p<.0001)$. For the control group, study times were similar for both categories $(t(5)=1.765, p=.14)$. 
In this experiment, the assumption that spreading activation occurred during mobilization was also tested. From this assumption, it can be deduced that mobilized items require less processing time to reach their setpoints than non-mobilized items within the category. In turn, these items would take less study time than items of the other, non-activated category since they could have received spreading activation during prior knowledge mobilization. In order to test this assumption, the 32 items were subdivided once more into three classes: (a) Mobilized items, (b) non-mobilized samecategory items, and (c) items from the non-mobilized category. Table 6.5 shows the results of this analysis.

Table 6.5

Mean study time per item, expressed in seconds, as a function of mobilization treatment. Local study time free and total study time fixed. The figures between brackets refer to the standard deviations.

Item category

Mobilization $\quad$ Mobilized Non-mobilized Non-category
treatment

Presidents group

$2.29(0.17)$

$3.50(0.17)$

$4.51(0.37)$

States group

$2.33(0.40)$

$3.65(0.23)$

$4.52(0.17)$

The two groups did not differ in amounts of study time spent $(F(1,10)<1)$. Different amounts of study time were spent on the three item types $(F(2,20)=$ $269.438, p<.0001)$. Identical patterns of study time allocation to these item types were found for the two subject groups $(F(2,20)<1)$.

The predictions of the cognitive set-point hypothesis were confirmed. Again, a linear effect was obtained $(F(1,10)=535.141, p=.0001)$. Nonmobilized same-category items were studied longer than mobilized items of this category. Yet, items of the non-mobilized category were processed longer than non-mobilized items of the category mobilized. These results replicate and extend the findings of Experiment 1 . They imply that, during mobilization, unvocalized items of the same category are pre-processed as a result of spreading activation, resulting in facilitation effects for non-mobilized items within the category activated.

Mean numbers of names of presidents and states correctly recalled by the three groups of subjects are presented in Table 6.6. 
Table 6.6

Mean number of correctly recalled items as a function of mobilization treatment. Local study time free and total study time fixed. The figures between brackets refer to the standard deviations.

Item category

\begin{tabular}{llll}
$\begin{array}{l}\text { Mobilization } \\
\text { treatment }\end{array}$ & Presidents & States & Means \\
\hline Presidents group & $7.17(0.98)$ & $9.83(0.98)$ & 8.50 \\
States group & $9.83(1.33)$ & $7.33(1.51)$ & 8.58 \\
Control group & $6.50(0.84)$ & $7.00(1.10)$ & 6.75
\end{tabular}

The three subject groups differed in mean number of items recalled $(F(2,15)=$ $5.646, p<.05)$. Identical recall scores were obtained for the two item categories $(F(1,15)=1.29, p=.27)$. The interaction of treatment and item category appeared to be significant $(F(2,20)=58.629, p<.0001)$. Overall, as was shown by means of Newman-Keuls comparisons (at the .05 level), the recall of the control group was poorer than that of the presidents group as well as the recall of the states group. Newman-Keuls comparisons (at the .01 level) also showed that the states group recalled more president names than both the presidents group and the control group, whereas the presidents group outperformed both the control group and the states group in recall of state names. Furthermore, the presidents group recalled more state names than president names $(t(5)=8.000, p<.001)$, whereas the opposite was found for the states group $(t(5)=11.18, p<.0001)$. Recall of the two item categories did not differ for the control group $(t(5)=1.168, p=.30)$.

Both the study time patterns and the recall results found in Experiment 2 provide support for the cognitive set-point hypothesis. The two experimental groups recalled significantly more items from the list than the control group due to the processing advantage acquired during mobilization. The spillingover effect can be clearly observed in both the recall data and the allocation of study time. The higher recall of the presidents group compared to the control group could be attributed to better recall of state names, whereas the states group outperformed the control group with respect to better recall of president names. Similarly, both experimental groups spent more time on nonmobilized items than on mobilized ones. 
However, it is important to note that in contrast to the predictions of the cognitive set-point hypothesis, the two experimental groups did not outperform the control group with respect to recall of items from the mobilized category. Newman-Keuls comparisons (at the .05 level) revealed that the presidents group did not recall more president names than the control group; the states group did not outperform the control group in recalling state names. The set-point hypothesis states that items from the mobilized category will be allocated less time but be recalled better compared to a control condition, owing to previous processing of that information. The recall data, however, do not support this prediction. It is unclear why the cognitive set-point hypothesis fails here. We will not elaborate on possible explanations at this point but return to the issue in the General Discussion section.

\section{EXPERIMENT 3}

In Experiment 3, both total and local study time were fixed. Each item on the list was presented for a fixed amount of time. It was assumed that the experimental groups would have a lead over the control group as a result of both pre-processing mobilized items and the spreading activation effect to other items within the same category. Thus, under fixed local study time conditions the experimental groups could be expected to reach the cognitive set-point for more items from the mobilized category compared to the control group. For the non-mobilized category, no differences in recall were to be expected since no "spilling over" of study time could take place. Therefore, the cognitive set-point hypothesis predicts that the experimental groups would outperform the control group for category items when local and total study time were fixed.

\section{SUBJECTS}

Subjects were 18 students of the University of Limburg, randomly assigned to one of the three conditions.

\section{PROCEDURE}

Experiment 3 only differed from the previous experiments in that both local and, hence, total study time were fixed: Subjects had no choice over allocation of study time time to the 32 items. Each item on the list appeared once on the screen for three seconds with an interval of one second between presentations. 


\section{RESULTS AND DISCUSSION}

Mean numbers of names of presidents and states correctly recalled by the three groups of subjects are presented in Table 6.7.

Table 6.7

Mean number of correctly recalled items as a function of mobilization treatment. Both local and total study time fixed. The figures between brackets refer to the standard deviations.

Item category

\begin{tabular}{lccc}
$\begin{array}{l}\text { Mobilization } \\
\text { treatment }\end{array}$ & Presidents & States & Mean \\
\hline Presidents group & $9.83(0.75)$ & $7.33(1.37)$ & 8.58 \\
States group & $7.83(1.84)$ & $10.33(1.97)$ & 9.08 \\
Control group & $6.83(0.41)$ & $7.17(1.17)$ & 7.00
\end{tabular}

The three groups of subjects differed in the mean number of items correctly recalled $(F(2,15)=4.646, p<.05)$. Again, similar recall scores were obtained for the president and state categories $(F(1,15)<1)$. A significant interaction of mobilization treatment and category was found $(F(2,15)=27.825, p<.0001)$. Subsequent Newman-Keuls comparisons showed that both the presidents group and the states group outperformed controls in overall recall. Furthermore, as was shown by Newman-Keuls comparisons (a the .01 level), the presidents group recalled more president names than either the control group or the states group, whereas the latter group outperformed the presidents group and the control group in the recall of state names. In addition, the presidents group recalled more president names than state names $(t(5)=$ $5.839, p<.01)$. On the other hand, the states group recalled more state names than president names $(t(5)=5.839, p<.01$ ), whereas the control group recalled the items of the two categories equally well $(t(5)<1)$.

As predicted, the experimental groups outperformed the control group in the totally fixed condition, an effect that could be attributed to better recall of items of the mobilized category. These data suggest that, indeed, recall is the result of an additive effect of time used for mobilization and time available at study as suggested by the cognitive set-point hypothesis. An additional 
analysis was carried out to test the proposition that the generation effect would also account for the better recall of the experimental groups. According to this hypothesis, self-generated items would be recalled better than items provided by the experimenter (McDaniel, Riegler, \& Waddill, 1990; Slamecka \& Graf, 1978). It could therefore provide an alternative explanation for the recall pattern found in Experiment 3. However, if the generation effect were applied to the present experiment, one would expect not only the mobilized category to be better remembered than the non-mobilized category, but, more importantly, mobilized items within a category to be better remembered than non-mobilized items within that same category.

To test the generation effect explanation, the president and state categories were split into two subclasses, each consisting of eight items: Items that were often mobilized (that had a high mobilization frequency) and items that were hardly ever mobilized (low mobilization frequency). Recall was compared for these four categories. So, if the generation effect hypothesis were to provide a valid explanation for the results of Experiment 3 , the experimental groups must outperform the control group solely for frequently mobilized items. Table 6.8 shows the results of this frequency analysis.

Table 6.8

Mean number of correctly recalled high mobilization frequency items and low mobilization frequency items by the experimental groups and the control group. Both local and total study time fixed.

Recall frequency

Mobilization frequency Experimental group Control group

Presidents

$\begin{array}{lll}\text { High } & 3.63 & 3.75 \\ \text { Low } & 3.75 & 1.38\end{array}$

States

$\begin{array}{lll}\text { High } & 4.75 & 4.25 \\ \text { Low } & 3.00 & 1.13\end{array}$

The resulls of this analysis show that the better recall of the experimental groups were not due to superior recall of items generated during the mobi- 
lization stage. In fact, the experimental groups and the control group performed equally well with respect to items with high mobilization frequencies. With respect to items that were infrequently mobilized, however, the experimental groups outperformed the control group. The presidents group recalled more president names that were only occasionally mobilized; the states group showed higher recall for state names with low mobilization frequencies. These differences were all statistically significant, indicating that the generation effect hypothesis does not provide sufficient evidence for the results of the present experiment.

\section{GENERAL DISCUSSION}

The cognitive set-point hypothesis assumes that people use an internal standard against which they measure when they have spent sufficient time on a certain unit of information and when the cognitive system can proceed to process subsequent units (Machiels-Bongaerts et al., 1990; Schmidt, 1982b, $1983 \mathrm{~b})$. This point of view has a number of implications for the allocation of study time to different items on a list and their subsequent recall. These implications were tested using the mobilization paradigm developed by Peeck (1982). The general idea is that mobilizing prior knowledge facilitates the processing of new relevant information by influencing time spent on different parts of that information. Since at mobilization some processing time has already been spent on some particular aspect of that information, when experimental subjects encounter that specific item again they reach the cognitive set-point for that information sooner than control subjects. Thus, mobilizing prior knowledge may influence the processing of subsequently presented information, inducing shorter processing times, better recall, or both, depending on the processing conditions.

In Experiment 1, two experimental groups mobilized names of either US presidents or states, whereas a control group mobilized names of composers. Subsequently, all subjects studied a list of names of presidents and states. Subjects were free to spend as much time on each of the items as they deemed necessary. As predicted by the cognitive set-point hypothesis, the control group displayed overall recall performance similar to that of both experimental groups but needed more study time to reach that level. Both experimental groups recalled equal numbers of items in each category. However, they allocated about half as much time to studying the items in the mobilized category, indicating that processing at mobilization curtailed processing time at subsequent presentation. The shorter study time in combination with the lack of differences in recall provided strong support for the existence of a cognitive set-point against which subjects measure whether sufficient time has been spent on an item in order to remember it. This cognitive set-point may vary 
depending on the importance of the task as perceived by the subjects or through external pressures. But by no means does the idea of a set-point imply that subjects study material to a $100 \%$ criterion. In Experiment 1 , for instance, subjects studied the material for about four minutes and recalled about 20 out of 32 items, although they were free to spend as much time as they liked.

When total study time was limited but local study time was free, as was the case in Experiment 2, the experimental subjects allocated more study time to items in the non-mobilized category, whereas the control subjects allocated an equal amount of time to items in both categories. In accordance with predictions, recall of the experimental groups exceeded recall of the control group, an effect that was entirely due to better recall performance of items from the non-mobilized category. Again, these results could be explained by assuming that subjects employ a cognitive set-point: Generation of prior knowledge causes certain items to be pre-processed, hence less time is needed for processing these same items to set-point when encountered in the list. The excess time is used to study items from the non-mobilized category more extensively.

Experiment 3 incorporating restriction of both local and, therefore, total study time extended the results of the previous experiments. The experimental subjects demonstrated better recall compared to the control group, an effect that was entirely due to better recall performance on the mobilized category items. These results indicated that mobilization of prior knowledge, even under conditions of fixed processing time, facilitates the recall of information relevant to that knowledge. This finding provides an additional indication that prior mobilization time is added to processing time at study resulting in superior recall of items from the mobilized category, as predicted by the theory of a cognitive set-point.

In addition, the present studies provide evidence that facilitative effects of mobilizing prior knowledge are by no means restricted to just the processing of mobilized items. In Experiments 1 and 2, analyses of study time allocated to non-mobilized items from the mobilized category suggested that mobilization effects extend to these items as well. Although experimental subjects took more time to study non-mobilized same-category items than mobilized items, they spent significantly less processing time on these items compared to study time spent on items from the non-mobilized category. In Experiment 3, it was shown that experimental subjects, in comparison to the control subjects, performed particularly well in the recall of non-mobilized items of the mobilized category, a finding also reported by Peeck (1982). These results seem to imply that at mobilization spreading activation affects items of the same category which although are not vocalized are processed nevertheless. This pre-processing causes these non-mobilized items from the 
mobilized category to reach the cognitive set-point quicker than items from the non-mobilized category, hence the shorter processing times and better recall under study time constraints (Machiels-Bongaerts \& Boshuizen, 1989). Observations of subjects during the experiment provide some anecdotal evidence for the idea of spreading activation at mobilization and its role in the processing of the list. For instance, a subject of the presidents group could not come up with the name "Roosevelt". He mentioned some features of this president (e.g., wheel-chair, talks with Stalin) but was unable to produce his name. When he was studying the list, the name "Roosevelt" appeared on the screen. Immediately, he buried his head in his hands, realizing that this was the item he failed to mobilize earlier. So, non-mobilized items of the category mobilized also seem to benefit from mobilizing prior knowledge since these items become more accessible as a result of spreading activation. In conclusion, the cognitive set-point hypothesis appears to account for the subjects" processing treatment of both mobilized and non-mobilized items of the mobilized category.

The alternative explanation for recall effects of mobilization was less convincing. The generation effect hypothesis (Slamecka \& Graf, 1978) predicts that items produced by the learner himself will be better recalled than items provided by the experimenter. In Experiment 1, no differences in recall were found between mobilized and non-mobilized items. In Experiment 2, items of the non-mobilized category were recalled more easily than subjectgenerated items. Even in Experiment 3, in which items from the mobilized category were recalled more easily, it was shown that the effect was entirely due to a better recall scores for non-generated items within the mobilized category. Of course, these different outcomes could be the result of using a somewhat different experimental manipulation. In that case, however, the present findings limit the generality of the generation effect to the generateread paradigm (McDaniel et al., 1990).

One could argue that the cognitive set-point phenomenon is the result of metamemory processes. One of the major skills involved in metamemory, in other words knowledge people have about their own memory, is monitoring. Glover, Ronning and Bruning (1990) define monitoring as "keeping track of one's progress as materials are committed to memory" (p. 105). This implies that subjects consciously and actively control the way they process information. The present experiments do not provide evidence in favor of such a proposition, yet they do not contradict it either.

However, although the cognitive set-point hypothesis accurately predicted patterns of study time allocation, prediction of recall patterns was less convincing. In Experiments 2 and 3, two anomalies were detected that need further scrutiny. 
To start with the easy anomaly: In Experiment 3 both local study time and total study time were fixed. It was predicted that the experimental subjects would perform well in recalling items for which there had been prior mobilization because of the processing advantage gained at mobilization. This prediction was confirmed. Further analysis revealed that the better recall was entirely due to superior recall of non-mobilized items within the mobilized category. (This effect was attributed to spreading activation received by these items at mobilization.) It is not clear, however, why the experimental subjects did not perform better in recalling specific items that had been mobilized. Their processing advantage for these items should have given them the edge over the control group as well. A closer examination of these items reveals that most of them are highly salient. Items like "Reagan", "Kennedy", "New York" and "California" must have been encountered and processed many times in the past, even by the Dutch students who volunteered as subjects in the present experiments. These items have become so easily accessible that studying them is not really necessary for recall. Therefore, for these highly salient items no recall differences would be expected between experimental and control subjects. This analysis would suggest further research with material that is less salient and whose mobilization would require more cognitive effort. Under those conditions, one may expect differences in recall for mobilized items as well.

More difficult to understand are the recall patterns produced in Experiment 2 . In that experiment, total study time was fixed but could be freely allocated to any item on the list. It was anticipated that experimental subjects would recall more items on the list than the control group (which they did) and that experimental subjects would spend relatively more time on studying items of the non-mobilized category (which they also did). However, the recall pattern of items from the mobilized category and the non-mobilized category as shown in Table 6.6 is difficult to interpret in the light of the cognitive set-point hypothesis. What is the explanation for the fact that items from the non-mobilized category are more easily recalled than items from the mobilized category? (Setting apart the performance of the experimental subjects from the control subjects who recalled both categories equally well.) The cognitive set-point hypothesis in itself would predict either (a) no differences if the cognitive set-point for all items had been reached, or (b) better recall of the mobilized category if there was insufficient time to reach the cognitive setpoints for the non-mobilized category. These predictions are clearly at variance with our findings, although they match findings of Peeck et al. (1982) and Schmidt (1982b) who used texts. They also found no differences between experimental subjects and controls for the mobilized information but facilitative effects for non-mobilized information. Three possible explanations come to mind. 
First, mobilizing prior knowledge could have interfered with memorization during the study phase. For instance, one subject in the presidents group mobilized "Bush". During free recall, this name came to his mind but he was not sure whether he had seen this item when studying the list or had mobilized it previously or perhaps both. To avoid confusion, subjects may have focused on items of the other non-activated category during the study stage. If a subject of the presidents group remembered the item "California" during the free recall test, he could be pretty sure that it was part of the item list. This attitude would reduce the number of recalled items of the mobilized category. This explanation, however, does not seem to be very plausible since the mobilized items matching the list were largely recalled in the three experiments: The mean correspondence between mobilized category items and recall of these items amounted to $83 \%$ for the presidents group and $80 \%$ for the states group.

A second possible explanation could lie in the novelty factor. The idea that during learning subjects focus specifically on new information is a recurrent theme in the learning and memory literature. As Kintsch (1980) puts it: "It's change, incongruity, surprise that leads to new learning" (p. 92). In fact, Peeck et al. (1982) also suggest that the shift in recall under mobilization conditions may be the result of the attraction of the new. Schmidt (1982b) speaks of a "boredom hypothesis" that may account for this shift. Subjects become bored at being confronted with the same study material again and therefore focus more attention on the new non-mobilized material. "This explanation, however, is unsatisfactory, because some of the items in the mobilized category were new or very nearly new to the experimental subjects, whereas some of the study material in the non-mobilized category must have been quite familiar, although it was not previously mobilized. Imagine a subject who mobilized names of presidents. To him the non-category item "California" was certainly not more unusual and, hence, more interesting than the non-mobilized same-category item "Polk". Why then, would he spend more time on processing the name of the state?

A final explanation might be that mobilization of prior knowledge lead subjects to overestimate the extent of their pre-existing knowledge and, thus, lower their set-points for that information. Lower cognitive set-points would necessarily result in allocating less processing time to items in the mobilized category, leaving more time for studying the non-mobilized category. This decrease in study time would go beyond predictions from the cognitive setpoint hypothesis, which fail to allow for lowered cognitive set-points, and would account for the recall pattern we found. The allocation of study time in Experiment 2 does not contradict this supposition. But what does militate against this hypothesis - and, for that matter against the novelty hypothesis is the result of Experiment 1 . In Experiment 1, subjects were free to spend as 
much time as they wished on the 32 items on the list and no differences in recall due to lowered cognitive set-points or novelty value were observed in that study.

Based on these observations, further studies of the mobilization of prior knowledge paradigm would seem to be appropriate. 
CHAPTER VII

SUMMARY AND IMPLICATIONS

\section{INTRODUCTION}

This thesis concerns the effects of prior knowledge activation on subsequent information processing and recall. During the last two decades, researchers have tried to study the impact of prior knowledge by manipulating this factor in various ways. Spilich et al. (1979), for instance, showed that subjects who had extended knowledge of baseball recalled more of a text about this topic than subjects whose knowledge in this domain was rather limited.

From this experiment as well as from a large number of other research studies, highlighted in Chapter I, the general conclusion can be drawn that prior knowledge has strong facilitative effects on information processing and recall. It should be resolved which mechanism accounts for these facilitation effects in order to be able to benefit from prior knowledge activation in fields such as education. A uniform answer to this question, however, seems difficult since a closer scrutiny of the studies reviewed in Chapter I showed that prior knowledge activation could result in different recall patterns, suggesting that different processing mechanisms underlie performance.

In Pichert and Anderson's (1977) study, for instance, prior knowledge was activated by means of assigning different perspectives from which a text should be read. The text described the adventures of two boys, playing hooky from school, in the house of one of them. This text contained information that was relevant either from a homebuyer's or from a burglar's perspective. The first group of subjects was told to read the text from the perspective of a burglar; the second group was instructed to read the text from a homebuyer's 
perspective. No reading perspective was directed to the third, control, group. After studying the text, subjects had to report everything they could remember from the text. The two perspective groups outperformed the control group in overall recall. These higher recall scores could be attributed to better recall of information relevant to the perspective previously assigned; the experimental groups and the control group did not differ in recall of information irrelevant to the reading perspectives. Similar results were found by Anderson and Pichert (1978), Goetz et al. (1983), Lintsen (1984) and Vonk (1986).

Peeck et al. (1982), on the other hand, found a recall pattern emerging from prior knowledge activation that was quite deviant from the pattern observed in Pichert and Anderson's (1977) study. Peeck et al. (1982) applied a different technique to activate prior knowledge. They requested an experimental group of elementary school children to mobilize knowledge (i.e., to generate all available knowledge about a particular topic) about the appearance and habits of the fox. This group and a control group, conducting a neutral mobilization task, read a short text about the appearance and habits of a fictional animal, the American heath fox. This text contained information congruent with the children's prior knowledge of the fox (congruous information), information contradicting this knowledge (incongruous information) and neutral information. Like in Pichert and Anderson's (1977) study, the experimental group outperformed the control group in overall recall as a result of prior knowledge activation. In the Peeck et al. study, however, the higher recall scores could be attributed to better recall of both incongruous and neutral information, whereas the experimental group did not outperform the control group in recall of congruous information. Johnson and Kieras (1983), Peeck (1982) and Schmidt (1982a) report similar findings.

Thus, in both groups of studies prior knowledge activation led to facilitative effects of prior knowledge activation; yet, they differed in the type of information benefiting from activating prior knowledge. In the study of Pichert and Anderson (1977) recall of information corresponding with the previously activated knowledge improved, whereas in the study of Peeck et al. (1982) information for which no activation took place was recalled best.

In the literature, two different cognitive mechanisms are found, each accounting for one of the recall patterns described.

The cognitive set-point hypothesis has been proposed to account for the recall patterns as observed in the Peeck (1982), Peeck et al. (1982) and Schmidt (1982a) studies. According to Schmidt (1983b), readers apply an internal setpoint against which they judge whether sufficient time has been allocated to a certain piece of information. The set-point level is supposed to be determined by an estimation of some minimum amount of understanding by the reader, his goals and motivation, and external pressures such as the expected conse- 
quences of not being able to reproduce adequately the information. If the setpoint level for a particular information unit has been reached, the reader will proceed to process new information, until the set-point for all information units has been reached, or time has run out. Schmidt assumes that, when subjects activate prior knowledge before studying a text, they already process this information to a certain extent. When they, subsequently, encounter the same or similar information in the text, they would require less time to reach the set-point for that information. If the total study time for a text is restricted, as, for instance, was the case in the studies mentioned earlier, a mobilization group would then remain more time to process non-mobilized information relative to a control condition. Consequently, better recall of this information could be achieved, resulting in overall higher recall.

The selective-attention hypothesis has been proposed by researchers who found that prior knowledge activation led to better recall of information corresponding with this knowledge (Anderson \& Pichert, 1978; Goetz et al., 1983; Lintsen, 1984; Vonk, 1986). According to this hypothesis, activating prior knowledge results in a selection process during information processing. Only information corresponding with the knowledge activated would be processed in depth, resulting in better recall of that information (Alba \& Hasher, 1983). This, in turn, would lead to overall higher recall scores.

Like the results they are based on, these hypotheses are quite contradictory. In Chapter I, we have tried to explain these differences. A first explanation might be the operationalization of prior knowledge activation. The apparently mutually exclusive results of the two series of experiments could have emanated from different techniques to activate prior knowledge (i.e., perspective assignment versus knowledge mobilization). However, a more appealing explanation might be the different nature of the prior knowledge activated in the two sets of studies, which may have resulted in different ways of information processing and recall patterns. Therefore, it is necessary to go deeper into the nature of the experiments and the process of knowledge acquisition as suggested by Schmidt and Boshuizen (1992) and Shuell (1990).

At first, when a person is still a novice in a domain, his knowledge is rather limited. It consists of a set of concepts with their interrelations. However, such a net will still contain many loose ends, while some of the relations have not yet been validated. With further knowledge acquisition and relevant experiences, new concepts are added, relations between concepts are included, while important concepts and links are differentiated from less important ones; this results in a network that can be traversed in many ways. In the end, this knowledge has been applied so often in interpreting events or other incoming information that radical structural changes have taken place. Now encapsulating concepts have been acquired embracing the detailed concepts started with. Simultaneously, schemata are formed. These knowledge 
structures group large sets of organized information about particular concepts. A schema represents the structure of a class of objects (or events, or procedures, or abstract concepts) according to a slot structure, where slots specify the values that the object has on various attributes. Schemata generalize a person's experiences with specific objects or events (Anderson, 1990).

People at different levells of expertise apply their knowledge in interpreting new information. Incoming information activates available knowledge; together they provide the mental representation of that information. Associative networks and schemata are activated in different ways. Concepts in associative networks are activated by spreading activation from concept to concept. When no direct link between a pair of concepts is available, which will happen more often at the earlier stages of expertise development, an inference has to be made. Furthermore, reasoning on detailed concepts takes more time than reasoning on encapsulating concepts that allow for abridged reasoning paths. Schemata, on the other hand, are activated as a whole. Once a schema has been activated, expectations about other attributes are generated. Hence, other schema relevant information automatically fills the slots of the schema, requiring a minimum of cognitive effort. This difference in knowledge activation results in differences in information processing.

In this continuum of knowledge acquisition, the selective-attention and the cognitive set-point research have their own niches. The selective-attention hypothesis has been investigated in domains the experimental subjects were well acquainted with and can be supposed to have schema-structured knowledge about (houses, burglary). The research on the cognitive set-point hypothesis, on the other hand, regards the early stages of knowledge acquisition; e.g., eleven year olds can not be supposed to have very detailed knowledge about different, exotic kinds of foxes. Consequently, different patterns of information processing may have been induced: Selecting and focusing slotfilling information versus activating concepts and constructing inferences. This difference might have resulted in different mental representations of the information and, hence, different recall patterns. In the Pichert and Anderson (1977) study, subjects who applied a homebuyer schema have focused on information relevant to that schema and subsequently better recalled that information than other information, and subjects focusing information relevant for their burglary schema recalled that information to the expense of other information. On the other hand, subjects in the Peeck et al. (1982) study activated knowledge about a subject they were only partially acquainted with. During subsequent information processing new relevant information could be focused and integrated into the initial knowledge base in order to extend it, resulting in better recall of information that had not been pre-activated.

This analysis can explain how such incongruous hypotheses like the selective-attention and the cognitive set-point hypothesis can co-exist and 
how confirming evidence for both could have been found. The purpose of the research presented in this thesis was to further pursue this explanation.

In Chapter II, a pilot study was reported. The goal of this study was two fold. First, the materials developed were tested. It was checked whether the text to be studied contained information corresponding with the knowledge previously activated by the subjects. Second, it was tested whether mobilization is an appropriate technique to activate prior knowledge that is either represented in a schema or in a less stable knowledge structure.

Subjects had to study a text, entitled "The consequences of the EEC fishery policies for Crando Douro" (Appendix D). This text contained three information categories: (a) EEC information in which the actions and consequences of the EEC fishery policies were described, (b) fish-farm information in which a possible solution for the problems stemming from these policies was outlined, and (c) tourism information in which references were made to the natural beauty of the village Crando Douro ("couleur locale") and its potential tourist abilities. Prior to text processing, half of the subjects had to mobilize orally everything they knew about the EEC fishery policies and their consequences, a topic much in the news at the time of testing; this experimental condition was called the EEC group. The specific knowledge activated by the EEC group was presumed to be limited and still unstable. The EEC group's prior knowledge was assumed to correspond with the EEC information in the text. A second group, the tourism group, was requested to mobilize orally general factors that could be of importance for a tour operator to decide whether a location was apt to become a new holiday resort. It was presumed that the tourism group's generic knowledge had evolved into a schema through many experiences in this domain. The tourism information in the text was assumed to fill slots of the tourism schema. In order to test whether the text indeed contained information corresponding with the knowledge activated by the two groups, subjects had to mark all information units relevant to their instruction during reading. After studying each sentence of the text in this way once, a distraction task had to be carried out. Finally, subjects had to report orally everything they could recall from the text.

The most important conclusions that could be drawn from this study were that (a) the EEC information corresponded with the EEC group's prior knowledge, (b) the tourism information matched the tourism group's prior knowledge, and (c) mobilization seemed to be an appropriate technique to activate prior knowledge, inducing either a selection or an extension process.

Allocation of study time plays a crucial role in both the cognitive set-point and the selective-attention hypothesis. Prior knowledge activation would determine the patterns of study time allocation during subsequent text pro- 
cessing. Different predictions, however, can be deduced from the two hypotheses regarding the information category receiving most study time. Thus, in order to test the predictions of the two hypotheses, highlighted in this thesis, both recall and allocation of study time would have to be registered. In Chapter III, these issues were taken into account. The predictions derived from the two hypotheses were tested in three studies.

In Experiment 1, the total amount of time available to study the text was fixed $\left(8^{\prime} 30^{\prime \prime}\right)$ but this time could freely be distributed over the various parts of that text. Prior to studying the text on a computer screen, the EEC group mobilized knowledge about the EEC fishery policies; the tourism group activated knowledge on what is important for tourism; a third, control, group mobilized knowledge about an unrelated topic. It was predicted that the EEC group would behave in accordance with the cognitive set-point hypothesis; the tourism group, on the other hand, would display a study time and recall pattern in line with the selective-attention framework.

The EEC subjects were expected to spend relatively less time on EEC policy information because they had already processed that information during mobilization and, therefore, would need less time to reach the setpoint for that information. It was also predicted that the EEC group would spend more time on new relevant fish-farm information because, compared to the control condition, more time would be available to do so. Recall patterns would be in agreement with the processing time patterns. By contrast, the tourism group was assumed to selectively attend to information in the text relevant to that "perspective". It was predicted that the tourism group would spend more time on processing tourism information than the other groups and, consequently, would show better recall of this information category.

The EEC group's patterns of study time allocation and recall, opposed to those of the control group, largely confirmed the predictions of the set-point hypothesis. Indeed, the control group studied EEC information longer than the EEC group, whereas the latter group spent most of the available time on new relevant fish-farm information. The recall patterns obtained matched these differences in information processing. The two groups did not differ in recall of EEC information; yet, the EEC group obtained higher recall scores for fish-farm information since this group, compared to the control group, had sufficient time to reach the cognitive set-point for this information category. An expected difference in overall recall did not materialize, however.

As predicted, the tourism group outperformed both the EEC group and the control group in recall of tourism information. The tourism group's recall score for tourism information exceeded the recall scores obtained for each of the remaining categories, which were all of more importance in the text itself. The major part of the study time available was spent on tourism information. Since the tourism group used only $69 \%$ of the totally available study time, the 
suggestion is not far-fetched that this group was selectively searching for relevant information, skipping all other information categories.

In Experiment 2 of Chapter III, subjects were free in allocating study time to the different text parts. The set-point hypothesis predicted no recall differences between the EEC group and the control group. The EEC group, however, would require less time than the control group to reach the set-point for the EEC information and, hence, would need less time overall. The tourism group, by contrast, would selectively attend only information relevant to the prior knowledge activated. Therefore, tourism subjects were expected to spend more time on tourism information and less on all other information categories. This pattern of study time allocation would lead to better recall of tourism information but lower overall recall scores. In addition, the selectiveattention hypothesis predicted that they would spend less time overall.

Overall, the EEC group recalled of the text as much as the control group but required less time to achieve that result. The difference in study time was located in the amount of time allocated to the EEC information. Here the control group spent significantly more study time than the EEC group. "These findings imply that the EEC subjects required less time for that category because they already had the opportunity to process similar information while mobilizing. Consequently, the EEC group could reach the set-point for EEC information sooner than the control group during text processing.

The tourism group's patterns of recall and study time obtained in the entirely free processing condition (Experiment 2) were almost identical to those of Experiment 1. Again, the tourism group excelled in recall of tourism information, which was studied longer than any of the remaining categories.

In Experiment 3 of Chapter III, each sentence was presented for a fixed amount of time. The set-point hypothesis predicted that the EEC subjects could only benefit from their processing advantage stemming from mobilization if they could freely allocate time to the various text parts. Therefore, in an entirely fixed condition, no differences between the EEC group and the control group were expected. The EEC group was no longer in a position to reduce time spent on mobilized information; neither could this group spend more time on new relevant information. Thus, since all categories would be processed equally long by the two groups, the EEC group's processing advantage would be nullified. The tourism group was assumed to mainly focus information corresponding with the knowledge activated, as far as possible within this fixed condition, leading to better recall of that information.

Indeed, the EEC group"s and the control group's overall recall did not differ. In fact, their recall patterns for the four categories were identical.

The tourism group's recall pattern hardly differed from those found in the previous studies. Apparently, the available study time was sufficient to select and process tourism information, resulting in superior recall of this in- 
formation category. Although the sentences of the EEC information, the fishfarm information and the residual information were presented for a fixed amount of time, this information was poorly recalled, suggesting that it could not be incorporated into the schema since it did not correspond with its slots.

The results obtained in Chapter III provide support for both the cognitive set-point hypothesis and the selective-attention hypothesis. The EEC group, reflecting the patterns as predicted by the cognitive set-point hypothesis, apparently tried to extend its limited knowledge by focusing on new relevant information. When only local study time was free (Experiment 1), its processing advantage stemming from prior knowledge mobilization resulted in higher recall scores than the control group. In an entirely free processing condition (Experiment 2), this led to a reduction of the amount of study time required to reach a total recall score similar to the control group's overall recall performance. This processing advantage was nullified in a completely fixed processing condition (Experiment 3 ). The tourism group's results provided support for the selective-attention hypothesis. In all processing conditions, slot-filling tourism information was predominant for both study time allocation and recall, implying that selective attention had occurred. Selective attention was particularly demonstrated in Experiment 3. Also in this fixed processing condition, slot-filling tourism information prevailed for recall although the remaining information categories, each of more importance in the text itself, had been presented for a fixed amount of time. Thus, the tourism groups overruled the so-called levels effect (Britton et al., 1979; Hustinx, 1985; Meyer, 1975), stating that important information in a text is recalled best.

Yet, although the subjects in the tourism groups seemed unable to resist the effect of schema activation, their results reported in Chapter III might still be accounted for by alternative explanations. While interpreting the tourism groups' findings, two difficulties were encountered. In Chapters IV and V, a number of studies were reported, trying to resolve these anomalies.

In Chapter $I V$, the assumption was tested that the tourism group's patterns of recall and study time allocation obtained in Chapter III could have emanated from a poor experimental instruction. In order to provide the subjects a reading goal, they had been told that a text contains important and less important information. It had been explained to the subjects that the goal of the research they participated in was to investigate whether important information can be discerned from unimportant information during text processing. This instruction may had had an undesirable impact on the tourism subjects; they could have interpreted "important" as "important regarding the knowledge previously mobilized". Thus, they might have assumed that they had to focus information corresponding with the prior knowledge activated during text processing. If this assumption would be accurate, the tourism groups" 
patterns of recall and study time allocation reported in Chapter III would not support the selective-attention hypothesis, but would merely confirm that the instruction applied had an unintended side-effect.

Three studies were reported which had to resolve whether schema mobilization induces a selection process during text processing beyond merely installing a reading goal. Subjects were run under two conditions: (a) Explicit instruction in which subjects were instructed to try to recall as much as possible of the text as a whole; and (b) implicit instruction, equaling the possibly ambiguous instruction used in the experiments described in Chapter III. If the explicit instruction groups would show patterns of recall and study time allocation identical to those of the implicit instruction groups, the effects found earlier could not be attributed to a faulty instruction.

The explicit instruction groups appeared to have been influenced by the modified instruction to some extent. When local study time was free (Experiments 1 and 2), the explicit instruction groups spent more processing time on information categories placed on high levels in the text's hierarchical structure than the implicit instruction groups. These patterns of study time allocation resulted in overall higher recall scores, which was attributable to better recall of EEC information and fish-farm information. Even in an entirely fixed processing condition (Experiment 3 ), this recall pattern emerged. Yet, recall of slot-filling tourism information was still predominant in all processing conditions. Most study time was spent on processing this category of information; consequently, recall of tourism information still prevailed.

In conclusion, despite the explicit instruction to recall as much as possible of the complete text, the explicit instruction groups largely displayed the same pattern of study time allocation as observed earlier: Selection of slotfilling tourism information, resulting in higher recall scores for this information category than for any of the remaining information categories in the text. These patterns of recall and study time allocation demonstrated the strong effects of schema activation on subsequent information processing and recall.

In Chapter $V$, a second difficulty encountered while interpreting the results of the tourism group in Chapter III was further investigated. The problem was that the tourism group did not seem to benefit from prior knowledge activation to the same extent as the EEC group. Like the EEC group, the tourism group was assumed to have a lead over the control group as a result of prior knowledge activation. Yet, the control group outperformed the tourism group. Selective attention was assumed to be responsible for this finding. However, the tourism group's overall lower recall may also have been due to the way in which the original text was constructed. The initial text was divided into three parts: EEC information, fish-farm information and tourism information, each comprising ten sentences or 22 propositions. One could ar- 
gue, however, that the EEC and the fish-farm information actually formed one text part. Although the EEC groups activated prior knowledge corresponding only with the EEC information, the fish-farm information could be considered as a logical extension of this information. Consequently, the text would have contained twice as much information relevant to the EEC group than to the tourism group. The overall lower recall scores of the tourism group may have originated from this difference in relevant information in the study text.

To test this assumption, the original text was rewritten. In the new text (Appendix III), the number of propositions relevant to the tourism group was extended, equaling the number of propositions available for the EEC subjects under the assumption that EEC and fish-farm information were interpreted as belonging to one category. The new text was applied in a free processing condition. Under the assumption that not a difference in processing strategy but simply the number of relevant propositions available could be held responsible for the findings of Chapter III, the EEC group and the tourism group were expected to benefit from prior knowledge activation largely to the same extent. Overall, the control group would require more time to obtain total recall scores similar to those of the EEC group and the tourism group.

However, despite the relatively increased recall of tourism information, the tourism group still showed lower overall recall than the EEC group and the control group. The latter two groups excelled in recall of EEC plus fishfarm information, whereas the tourism group recalled the tourism information best. As expected, the control group required overall more time to study the text than the EEC group and the tourism group. Remarkably, the tourism group, like the EEC group and the control group, spent most time on EEC and fish-farm information; yet, recall of this information remained poor. Thus, the tourism group appeared to have attempted to follow the explicit instruction to try to recall as much as possible of the complete text, reflected by the pattern of study time allocation. However, despite the huge amount of time allocated to the EEC and fish-farm information, this information was hard to recall, probably due to the fact that it did not fill slots of the activated schema.

In Chapter VI, an attempt was made to extend the findings of Chapters II and III, supporting the cognitive set-point hypothesis, to item lists in order to test the generality of this hypothesis. Three studies were described in which available study time was manipulated in the same way as in the studies of Chapter III. Instead of a text, subjects were presented with an item list, containing 16 US president names and 16 names of states of the Union in random order. In each study, three groups participated. Subjects either mobilized as many names as possible of US presidents (presidents group), of states of the Union (states group) or of composers (control group). The two experimental groups, the presidents and the states group, were expected to benefit 
from mobilizing relevant prior knowledge. The facilitation effects emerging from prior knowledge activation would be either located in overall recall or in total amount of time required for list processing, depending on the processing condition. Indeed, if totally available study time was fixed which could freely be allocated to the 32 items (Experiment 2), the experimental groups outperformed the control condition in overall recall. Both the presidents group and the states group required less time to study items of the mobilized category; relatively more time remained to process items of the other non-activated category. Consequently, the experimental groups were able to reach the setpoint for more items of the non-activated category than the control group, resulting in overall higher recall. On the other hand, if subjects were entirely free to allocate study time to each item in the list (Experiment 1), the control group could make arrears by spending more time on list processing. No recall differences were found between the experimental groups and the control group. Since the experimental groups needed less time to reach the set-point for items in the mobilized category as a result of prior knowledge mobilization, the control group required substantially more time to achieve recall performances similar to those of the experimental groups.

Contrary to the predictions of the set-point hypothesis, the two experimental groups also obtained higher recall scores than the control group in an entirely fixed processing condition (Experiment 3 ). The set-point hypothesis predicted that the experimental subjects could only benefit from prior knowledge mobilization if they could influence study time allocation. The amount of time spent on mobilized information would be reduced, resulting in relatively more time for non-activated information. This processing advantage would be nullified in a fixed processing condition; consequently, no recall differences could emerge. Yet, in this study the two experimental groups outperformed the control subjects, which could be attributed to better recall of the mobilized item category. This was attributed to spreading activation taking place during mobilization. Activation may have spread from items mobilized to items within the category assigned which had not been mobilized, increasing their accessibility level. For instance, the non-mobilized item "Johnson" could have received spreading activation from the mobilized item "Kenned $y^{\prime \prime}$. If spreading activation does occur while mobilizing items of a certain category, one would expect that non-mobilized items of the same category would also benefit from prior knowledge activation, but to a lesser extent than mobilized items of that particular category. Additional analyses supported this assumption. In conclusion, the cognitive set-point hypothesis appears to account for the subjects' processing behavior of both mobilized and nonmobilized items of the mobilized category, provided that the assumption of spreading activation occurring at mobilization is integrated in this theory. Remarkably, no effects of spreading activation did emerge in text processing 
(Chapter III) although it is considered to be a key element in the constructionintegration model of text processing (Kintsch, 1988).

\section{DISCUSSION AND CONCLUSIONS}

The first conclusion that could be drawn from the findings reported in this thesis is that they provide considerable support for the idea that humans, while processing information, use a cognitive standard for comprehension against which they judge whether sufficient time has been allocated in order to meet that standard. Results showed that, as predicted, prior knowledge mobilization, under appropriate conditions, could induce subjects to spend less time on studying similar information when subsequently encountered in a text. Alternatively, subjects allocated more study time to information that might extend their knowledge base beyond what is already known. The differential study time allocation of the experimental (EEC) group versus the control group in Chapter III is congruent with the idea that the processing of information is subject to a cognitive set-point which takes into account the whole processing cycle including mobilization. The studies obtained in Chapter VI generally confirmed and extended these conclusions.

A second major finding is that the two hypotheses under study, the selectiveattention and the cognitive set-point hypothesis, are not by definition mutually exclusive. Both hypotheses could reflect two different phases in the knowledge acquisition process as suggested by Schmidt and Boshuizen (1992).

The set-point hypothesis may apply if prior knowledge is activated that is presumed to be limited and still unstable. The nature of this knowledge would induce an extension process during information processing. New relevant information would be focused to extend the limited knowledge base, which in turn would result in the recall patterns as predicted by the set-point hypothesis: Better recall of information not previously activated.

The selective-attention framework, on the other hand, may apply if knowledge is activated that is assumed to have evolved into a rather stable knowledge structure through many experiences in a particular domain. Activating such a schema would instigate a selection process during text processing. Information filling slots of the schema would be selected, which would lead to the recall pattern as predicted by the selective-attention hypothesis: Superior recall of information matching the activated knowledge.

However, although the EEC groups' and the tourism groups' patterns of recall and study time allocation were in line with the theory of knowledge acquisition outlined, the reader should bear in mind that in this thesis we have not been able to provide direct evidence of the nature of the cognitive structures involved in the process of knowledge acquisition. 
A third conclusion is that during mobilization more is activated than the knowledge actually produced by the subjects. In the third study in Chapter VI, facilitation effects of prior knowledge mobilization were found in a fixed processing condition. The experimental groups outperformed the controll condition in overall recall of list items, which was attributable to better recall of the mobilized item category. The higher recall scores under those processing conditions were ascribed to spreading activation during mobilization. Non-mobilized items within the activated category could have received spreading activation of mobilized items, increasing the accessibility level of non-mobilized items. This could account for the higher recall scores for nonmobilized items. Additional analyses supported this assumption.

The question whether spreading activation occurs in text processing remains unanswered since no facilitation effects were found in an entirely fixed processing condition, using a text (Chapter III). According to Kintsch's (1988) construction-integration model, however, spreading activation plays an essential role in text processing.

A fourth conclusion regards the generalization of the generation effect (McDaniel et al., 1990; Slamecka \& Graf, 1978), which could provide an alternative explanation for the recall pattern obtained in the final study of Chapter VI. The generation effect refers to the phenomenon that self-generated items are recalled better than items provided by an experimenter. If this hypothesis would apply to the third study, one would expect not only the mobilized category to be remembered better than the non-mobilized category, but, more importantly, also mobilized items within a category to be recalled better than non-mobilized items of that same category. However, an additional analysis revealed quite the opposite. The experimental groups' better recall performances on the mobilized category items could entirely be attributed to better recall of non-generated items within the mobilized category. These deviant outcomes could be attributed to differences in experimental manipulations; however, the present findings would still limit the generality of the generation effect to the generate-read paradigm.

\section{SUGGESTIONS FOR FURTHER RESEARCH}

The research presented in this thesis resulted in a number of new questions which need further investigation.

A problem encountered is that the knowledge mobilization procedure is difficult to control. Information processing seems to be subject to a cognitive setpoint taking into account the whole processing cycle including mobilization. Thus, to adequately test the impact of prior knowledge activation on subse- 
quent information processing, time spent on certain information units during mobilization should be registered. This, however, does not seem to be so easy. For instance, while mobilizing US presidents, a subject pauses. Should the time a subject uses to reflect upon another member of the assigned category be considered as pre-processing time for that particular item? What about subjects who generate a certain item twice? Should these mobilization times be added as being the pre-processing time of that particular item? In other words, mobilization is still hard to measure.

To resolve these problems, the mobilization procedure might be replaced by a rereading task (Johnson \& Kieras, 1983; Raney, 1993). In this task, prior knowledge is activated by a first pass reading of that part of the text for which activation is to take place. The text to be studied contains this previously activated information (either verbally or conceptually identical) as well as new, non-activated information. For both the text in first pass and the study text, processing time required for each information unit will be registered. The major advantage of this rereading paradigm is that a better estimate can be made of the total amount of time spent on the various parts of the text.

A variant of the experimental procedure outlined could solve the mystery of spreading activation in text processing. To test whether spreading activation occurs in text processing - but is masked - or not, a text would have to be construed containing (a) information matching the activated knowledge, (b) new, non-activated information, and (c) information that had not been activated but that could be assumed to have received spreading activation during prior knowledge activation. The set-point hypothesis would predict patterns of recall and study time identical to those found in Chapter VI for item lists. If these predictions would be confirmed, "real" facilitation effects emerging from the activated knowledge itself would be shown in text processing.

Despite these measurements, prior knowledge remains a factor hard to control. To some extent, the investigator has to make an educated guess about the nature and extent of subjects' prior knowledge, based on probing similar subjects in pilot studies. And even then, there may be considerable variability among subjects. 1 For instance, suppose the rereading task is applied to the item list of Chapter VI. Names of eight presidents could serve to activate prior knowledge. Subsequently, a list of 16 president names is presented; eight of them are identical to the activation list. Although prior knowledge activation is controlled because all subjects activate the same information, the possibility remains that subject $A$ is familiar with more items of the non-activated list

1 This did not appear to be the case for the tourism subjects, who hardly differed in factors of the holiday schema, neither in number nor in types. Furthermore, Barsalou (1991) and Figler. Weinstein, Sollers and Devan (1992) found the same elements of the tourism schema. 
than subject $B$; in other words, despite controlling the activated information, subjects can differ in prior knowledge about the non-activated information.

In order to resolve this problem, prior knowledge should be acquired in an experimental setting. As outlined earlier, the acquisition of knowledge can be viewed as the evolution of limited specific knowledge into generic knowledge, represented in a schema. Controlling this process would enable a more direct test of the hypothesis postulated in this thesis that the cognitive setpoint hypothesis and the selective-attention hypothesis both may apply on different knowledge levels. One group of subjects could be provided with a large number of trials in which particular knowledge actively has to be applied; in this way, the initial knowledge base could develop into a schema. A second subject group would only possess the theoretical knowledge. Since this knowledge would not be applied to a variety of situations no schematic structure could evolve. The patterns of recall and study time allocation shown by the first group under the various manipulations of available study time are predicted to be in line with the predictions of the selective-attention hypothesis, whereas the patterns of the second subject group are expected to reflect the assumptions of the cognitive set-point hypothesis.

Although the cognitive set-point hypothesis enables quite specific predictions about subjects' patterns of recall and study time allocation, it does not really explain these phenomena since this hypothesis is not suitably embedded in more general theories of text processing and comprehension.

In a recent discussion, Walter Kintsch ${ }^{1}$ suggested that the set-point phenomena might be explained at a deeper level by his construction-integration model of text processing (Kintsch, 1988). According to Kintsch, text comprehension can be understood as a largely bottom-up process. He assumes that knowledge can be conceived as an associative network of nodes (mostly concepts) and interrelations. When a reader attempts to understand a sentence in a tex $t_{s}$ words or phrases will activate corresponding nodes and activation will spread to other related nodes. In this way, a pool of knowledge is activated that may or may not be relevant to the task of understanding at hand (construction phase). With further reading, this pool of knowledge will be reduced. Processing subsequent information will activate more concepts; this contextual information limits the number of candidates giving meaning to the previous information. In other words, the new information deactivates irrelevant knowledge. During the subsequent integration phase a coherent representation of the sentence is built by integrating knowledge from earlier cycles of activation into that of later cycles. This kind of information processing

1 This discussion followed the presentation of the paper "Cognitive Set-Points in Text Processing* by M. Machiels-Bongaerts, H. Schmidt and H.P.A. Boshuizen, (1993, April) at the AERA meeting in Atlanta, GA. 
would continue until the emerging representation satisfies constraints set by the task. The parameter " $h$ " (for "harmony") in the construction-integration model of Kintsch (1988) indicates that this relaxation process can reach an optimum at which the representation has become stable.

Mobilizing relevant prior knowledge preceding text processing could speed up the construction phase. As a result of prior knowledge mobilization some context is already provided in light of which the concepts in the text should be understood. Therefore, irrelevant knowledge may be faster deactivated and less cycles of activation may be necessary in order to create coherence. Consequently, a reader may proceed through the study text at a faster rate.

According to Kintsch, a cognitive set-point may be construed as the optimum value of " $h$ ", given the task constraints. The set-point determines what level of coherence is acceptable in order to proceed to a next sentence or paragraph. This seems to be intuitively attractive because it clarifies that people can glance at a text to get an impression of its contents or study the same text for future information retrieval. The reader's intention determines the set-point level and, hence, defines which degree of coherence is adequate.

In order to test Kintsch"s assumption that cognitive set-points may be construed as optimum values of " $\mathrm{h}$ ", the data produced in the studies presented in this thesis should be modelled to the results of simulations using the architecture developed by Kintsch and his associates (Dellarosa, 1986). Combining the studies suggested in the previous paragraphs and these simulations would provide an opportunity to test the generalizibility of the setpoint hypothesis since different techniques would be applied to activate prior knowledge.

\section{EDUCATIONAL IMPLICATIONS}

From the results obtained in this thesis, a number of educational implications can be derived.

The activation of pre-existing knowledge preceding a new study phase is frequently recommended in educational research (Gagné, 1974; Mayer, 1979, 1985). However, transferring the findings in this thesis to education, it could be concluded that prior knowledge mobilization would be a rather ineffective educational activity to employ. In "normal" learning settings an appropriate schema is not available to the students; in other words, their knowledge in specific domains is still limited and it has not extensively been used in a variety of situations. Therefore, the predictions of the cognitive set-point hypothesis would apply to education. An "entirely" free processing condition is another feature of every day learning; students are (at least theoretically) free 
to allocate as much processing time as they like to the new study material. Under such processing conditions, prior knowledge mobilization would lead to a reduction of the study time required to reach the set-point, whereas the recall performances would not exceed the scores in a learning setting without prior knowledge activation. In practice, thus, the effects of mobilizing relevant prior knowledge on information processing and recall would be rather limited. In fact, the net effects stemming from prior knowledge mobilization might even be nihil. Since mobilization takes time, the sum of time spent at mobilization and the amount of time required for text processing could add up to the amount of study time used in a learning setting without mobilization. In that case, prior knowledge mobilization would not be efficient at all. Thus, if prior knowledge mobilization (i.e., generating pre-existing knowledge) is merely a stimulating recall event (Gagné, 1974), accessing earlier acquired knowledge which can serve as a "hat-rack" to hang upon the new information, it would be a rather inefficient educational activity.

Notwithstanding that, facilitating effects could emerge if prior knowledge activation results in restructuring the initial knowledge base. Preprocessing events like advance organizers (Ausubel \& Youssef, 1963; Mayer, 1980, Experiment 1), metaphors (Niederhauser \& Reynolds, 1991; Paris, 1988), visual representations or diagrams (Beveridge \& Parkins, 1987; Gick \& Holyoak, 1980, 1983) and analogies (Simons, 1984; Vosniadou \& Schommer, 1988) require an active manipulation of the existing prior knowledge. Restructuring the initial knowledge base may lead to facilitation effects which go beyond time-saving effects with respect to processing new information until set-point. These pre-instructional strategies also influence the way in which the information to be subsequently studied is processed. In that case, prior knowledge activation could result in better (recall) performances even in an entirely free processing condition.

If prior knowledge activation is structurally integrated in education, students should be guarded against focusing merely one particular information category in the study text as a result of this task, largely ignoring the remaining information in that text. This pattern of study time allocation reflects the predictions of the selective-attention hypothesis. This way of selective information processing would lead to better performances for information matching the activated prior knowledge, but not to overall higher scores. Willems (1987), for instance, demonstrated that questions prior to the new learning task did result in better scores for information providing an answer to those questions; however, this activation task did not lead to higher performances for the remaining information parts of the text. Rickards and Denning (1979) also report negative effects of questions incorporated in the study text on overall performance. 
As pointed out earlier, prior knowledge mobilization would not be as effective as other pre-instructional strategies. The findings reported showed that subjects compensate by simply spending less time on text processing; in other words, mobilization would merely result in a reduction of the study time required to reach the cognitive set-point.

However, by raising the set-point level "true" facilitation effects of prior knowledge activation could emerge even in an entirely free processing condition; l.e. better performances instead of merely time-saving effects. The degree of text coherence acceptable to the learner, established by his learning goal, determines the amount of time spent on the new study material. Prior to studying new information, the learner (unconsciously) determines the setpoint level, which in turn dictates his processing strategy. A number of factors influence the choice of the appropriate strategy. First, the learner's expectations with respect to (desired) learning outcomes guide the way in which attention is paid to various information parts of the text (Andre, 1979; Reynolds \& Shirey, 1988). Does he have to know this information by heart or should he be able to reproduce the core of the text? Second, the learner's aspiration level influences the processing strategy. Third, the choice of the processing strategy is determined by the learner's interest (Anderson, 1982).

An active manipulation of (one of) these factors could raise the level of the cognitive set-point. The first factor, estimation of the desired learning outcomes, could be influenced by informing students prior to studying a text what kind of test they subsequently have to administer (Nungester \& Duchastel, 1982). Information that is considered to be relevant in light of the task at hand is given more attention (Rothkopf \& Billington, 1979; Van Hout Wolters, 1986; Willems, 1987). For instance, if the learner knows that he has to present his newlly acquired knowledge to his peers opposed to the slim chance to be tested in class, the set-point level could be raised as a result of this social pressure. $\mathbb{1}$ The second factor, the learner's ambition, is difficult to influence. In education, the importance of the information to be studied could be emphasized in order to reach a certain goal. Depending on the learner's aspiration, the choice of the processing strategy, and thus the set-point level, could be modified. Also the third factor, the learner"s interest, is hard to manipulate since this aspect resides within the individual learner. However, this factor could be accommodated by selecting or constructing study material that is appealing to the learner. By presenting study material that is in the news, well-structured, up-to-date and illuminated by pictures and examples, the learner's motivation, and, in turn, the set-point level, might be raised.

\footnotetext{
1 Schmidt and Gijselaers (1990) demonstrated that in problem-based learning student performance is determined by student group functioning (through amount of study time spent on processing relevant information), which in turn is largely determined by the amount of prior knowledge activated prior to the study phase.
} 


\section{SAMENVATTING EN IMPLICATIES}

INLEIDING

In dit proefschrift staat de invloed van voorkennisactivatie op het verwerken en het onthouden van informatie centraal. Gedurende de laatste twee decennia hebben onderzoekers getracht de effecten van voorkennis te bestuderen door deze factor op verschillende manieren te manipuleren. Spilich et al. (1979), bijvoorbeeld, toonden aan dat proefpersonen die een uitgebreide kennis van baseball bezaten meer onthielden van een tekst over dit onderwerp dan proefpersonen die van dit onderwerp weinig afwisten.

Uit dit experiment alsmede uit een groot aantal andere onderzoeken, die in Hoofdstuk I belicht worden, kan de algemene conclusie getrokken worden dat voorkennis sterk faciliterende effecten heeft op het verwerken en onthouden van informatie. Om te kunnen profiteren van het activeren van voorkennis in terreinen zoals onderwijs dient opgehelderd te worden welk mechanisme een verklaring kan bieden voor deze effecten. Een eensluidend antwoord op deze vraag lijkt echter onmogelijk sinds een nader onderzoek van de in Hoofdstuk I besproken studies aantoonde dat voorkennisactivatie tot verschillende recallpatronen kan leiden, hetgeen suggereert dat aan deze patronen verschillende verwerkingsmechanismen ten grondslag liggen.

In het onderzoek van Pichert en Anderson (1977), bijvoorbeeld, werd voorkennis geactiveerd door het toewijzen van verschillende perspectieven vanwaaruit een tekst gelezen moest worden. De tekst beschreef de avonturen van twee spijbelende jongetjes in het huis van één van hen. Deze tekst bevatte informatie die relevant was of vanuit het perspectief van een huizenkoper of vanuit het perspectief van een inbreker. De eerste groep proefpersonen werd 
opgedragen de tekst te lezen vanuit het inbreker-perspectief; de tweede groep werd geinstrueerd de tekst te lezen vanuit het huizenkoper-perspectief. De derde groep, de controlegroep, werd geen leesperspectief opgelegd. Na het bestuderen van de tekst moesten de proefpersonen alles rapporteren wat ze zich konden herinneren van de tekst. De beide perspectiefgroepen behaalden in totaal hogere recallscores dan de controlegroep. Dit kon worden toegeschreven aan het beter onthouden van informatie die relevant was ten opzichte van het toegewezen perspectief; de experimentele groepen en de controlegroep verschilden niet in het onthouden van informatie die irrelevant was ten opzichte van de beide perspectieven. Anderson en Pichert (1978), Goetz et al. (1983), Lintsen (1984) en Vonk (1986) boekten vergelijkbare resultaten.

Peeck et al. (1982) vonden echter een recallpatroon, resulterend uit voorkennisactivatie, dat volledig afweek van het patroon dat geconstateerd werd in de studie van Pichert en Anderson (1977). Peeck et al. (1982) pasten een andere techniek toe om voorkennis te activeren. Een groep basisschoolleerlingen werd gevraagd kennis te mobiliseren (i.e., alle beschikbare kennis omtrent een bepaald onderwerp te genereren) met betrekking tot het uiterlijk en de gewoonten van de vos. Deze experimentele groep en een controlegroep, die een neutrale mobilisatietaak verrichtte, lazen een korte tekst over het uiterlijk en de gewoonten van een verzonnen dier, de Amerikaanse heidevos. Deze tekst bevatte informatie die congruent was met de voorkennis van de leerlingen (congruente informatie), informatie die in strijd was met de voorkennis van de proefpersonen (incongruente informatie) en neutrale informatie. Evenals in het onderzoek van Pichert en Anderson (1977) bleek de totale recallscore van de experimentele groep de score van de controlegroep te overschrijden. In het experiment van Peeck et al. konden deze hogere recallscores toegeschreven worden aan het beter onthouden van zowel incongruente als neutrale informatie, terwijl de experimentele groep en de controlegroep niet verschilden in het onthouden van congruente informatie. Johnson en Kieras (1983), Peeck (1982) en Schmidt (1982a) rapporteren vergelijkbare bevindingen.

In beide groepen experimenten werd dus geconstateerd dat voorkennisactivatie tot faciliterende effecten leidde; het type informatie dat van voorkennisactivatie profiteerde verschillde echter. In het onderzoek van Pichert en Anderson (1977) werd informatie die correspondeerde met de eerder geactiveerde kennis beter onthouden, terwijl in het onderzoek van Peeck et al. (1982) niet-geactiveerde informatie het best onthouden werd.

Twee verschillende cognitieve mechanismen worden in de literatuur beschreven, die ieder een verklaring bieden voor één van de geconstateerde recallpatronen. 
De cognitieve set-point hypothese werd voorgesteld als verklaring voor de recallpatronen die in het onderzoek van Peeck (1982), Peeck et al. (1982) en Schmidt (1982a) gevonden werden. Volgens Schmidt (1983a) hanteren lezers een interne standaard waaraan zij afmeten of voldoende tijd besteed is aan een bepaald informatiedeel. Verondersteld wordt dat het niveau van de setpoint bepaald wordt door een inschatting van de lezer omtrent een minimaal comprehensieniveau, de motivatie en doelen van de lezer en externe druk zoals de verwachte consequenties wanneer men niet in staat blijk te zijn de informatie adequaat te reproduceren. Wanneer de set-point voor een bepaald deel van de informatie is bereikt, zal de lezer doorgaan met het verwerken van nieuwe informatie totdat de set-point voor alle informatiedelen van de tekst bereikt is of de beschikbare hoeveelheid studietijd verstreken is. Schmidt veronderstelt dat tijdens voorkennisactivatie, voorafgaande aan het bestuderen. van een tekst, deze informatie al gedeeltelijk verwerkt wordt. Wanneer vervolgens de studietekst dezelfde of vergelijkbare informatie bevat, zou relatief minder tijd nodig zijn om voor deze informatie de set-point te bereiken. Wanneer de totale studietijd voor een tekst gelimiteerd is, zoals bijvoorbeeld in de eerder genoemde onderzoeken, zou een mobilisatiegroep meer tijd resteren voor de verwerking van niet-gemobiliseerde informatie dan een controlegroep. Hierdoor zou die informatie beter onthouden kunnen worden, leidend tot in totaal hogere recallscores.

De selectieve-aandachtshypothese werd voorgesteld door onderzoekers die constateerden dat voorkennisactivatie leidde tot het beter onthouden van informatie, corresponderend met deze kennis (Anderson \& Pichert, 1978; Goetz et al., 1983; Lintsen, 1984; Vonk, 1986). Volgens deze hypothese resulteert voorkennisactivatie in een selectieproces tijdens daaropvolgende informatieverwerking. Alleen informatie die correspondeert met de geactiveerde kennis zou grondig bestudeerd worden, hetgeen zou resulteren in hogere recallscores voor die informatie (Alba \& Hasher, 1983). Dit zou wederom leiden tot in totaal hogere recallprestaties.

Deze hypothesen zijn, net als de bevindingen waarop ze gebaseerd zijn, nogal tegenstrijdig. In Hoofdstuk I werd getracht deze verschillen te verklaren. Een eerste verklaring zou gelegen kunnen zijn in de operationalisatie van voorkennisactivatie. De ogenschijnlijk tegenstrijdige resultaten van de beide groepen experimenten zouden kunnen voortvloeien uit verschillende technieken om voorkennis te activeren (e.g., het toewijzen van een perspectief versus het mobiliseren van kennis). Een aantrekkelijkere verklaring zou echter betrekking kunnen hebben op de aard van de voorkennis, die geactiveerd werd in de beide reeksen van experimenten. Verschillende typen kennis zouden geactiveerd kunnen zijn, hetgeen geleid zou kunnen hebben tot verschillende informatieverwerkingsprocessen en recallpatronen. Teneinde deze assumptie nader toe te lichten zal dieper ingegaan moeten worden op de experimen- 
ten zelf en het proces van kennisverwerving zoals voorgesteld door Schmidt en Boshuizen (1992) en Shuell (1990).

In het begin wan dit proces, wanneer iemand nog een noviet is in een bepaald domein, is zijn of haar kennis nogal beperkt. Deze kennis bestaat uit een serie concepten en hun onderlinge relaties. Zo'n netwerk zal echter nog steeds "losse eindjes" bevatten, terwijl sommige relaties nog niet gevalideerd zijn. Met toenemende kennis en relevante ervaringen worden nieuwe concepten en onderlinge relaties toegevoegd, terwijl belangrijke concepten en relaties onderscheiden worden van minder belangrijke. Dit resulteert in een netwerk dat op verschillende wijzen doorkruist kan worden. Uiteindelijk is deze kennis zo vaak gebruikt om gebeurtenissen of andere relevante binnenkomende informatie te interpreteren dat radicale structurele veranderingen zijn opgetreden. Op dat moment ontstaan geëncapsuleerde concepten die de gedetailleerde concepten omvatten waarmee oorspronkelijk gestart werd. Tegelijkertijd worden schema's gevormd. Grote georganiseerde brokken informatie omtrent bepaalde concepten worden in deze kennisstructuren gegroepeerd. De structuur van een klasse van objecten (of gebeurtenissen, of procedures, of abstracte concepten) wordt in een schema gerepresenteerd als een structuur van slots, waarbij een slot de waarden specificeert die de eigenschappen van een object kunnen aannemen. Schema's generaliseren de ervaringen die iemand heeft met specifieke objecten of gebeurtenissen (Anderson, 1990).

Mensen met verschillende niveaus van expertise gebruiken hun kennis bij het interpreteren van nieuwe informatie. Binnenkomende informatie activeert de beschikbare kennis; samen vormen zij de mentale representatie van die informatie. Associatieve netwerken en schema's worden op verschillende manieren geactiveerd. Concepten in een associatief netwerk worden geactiveerd doordat spreidende activatie optreedt van het ene concept naar het andere concept. Wanneer er geen directe verbinding bestaat tussen twee concepten, hetgeen vaker gebeurt in de beginfase van expertise-ontwikkeling, zal een inferentie gemaakt moeten worden. Het redeneren op grond van gedetailleerde concepten vergt bovendien meer tijd dan het redeneren op grond van geëncapsuleerde concepten die verkorte redeneerstappen mogelijk maken. Een schema wordt daarentegen als één geheel geactiveerd. Wanneer een schema geactiveerd is, worden verwachtingen gevormd omtrent de overige attributen van dit schema. De slots van het schema worden dus automatisch gevuld met andere informatie die relevant is ten opzichte van het schema, hetgeen nauwelijks cognitieve inspanning vereist. Dit verschil in het activeren van kennis resulteert in verschillende informatieverwerkingsprocessen.

In dit continuum van kennisverwerving neemt het onderzoek van de selectieve-aandachtshypothese en dat van de cognitieve set-point hypothese elk een eigen plaats in. Het onderzoek verricht in het kader van de selectieveaandachtshypothese had betrekking op domeinen waarmee de experimentele 
groepen proefpersonen vertrouwd waren; aangenomen kan worden dat zij hieromtrent schematisch gestructureerde kennis bezaten (huizen, inbraak). Het onderzoek van de cognitieve set-point hypothese richtte zich daarentegen op de beginfase van kennisverwerving; e.g., 11 -jarigen kunnen niet geacht worden zeer gedetailleerde kennis te bezitten omtrent verschillende soorten uitheemse vossen. Dit zou geleid kunnen hebben tot het oproepen van verschillende processen van informatieverwerking: Het selecteren en aandachtig bestuderen van slotvullende informatie versus het activeren van concepten en het vormen van inferenties. Dit onderscheid kan geleid hebben tot verschillende mentale representaties van de informatie en dus tot verschillende recallpatronen. In de studie van Pichert en Anderson (1977) richtten proefpersonen die een huizenkoper-schema toepasten zich op informatie die relevant was voor dat schema, waardoor die informatie beter onthouden werd dan de overige informatie; proefpersonen die zich concentreerden op informatie die relevant was voor hun inbreker-schema blonken uit in het onthouden van deze informatie, hetgeen ten koste ging van de overige informatie. De proefpersonen in het onderzoek van Peeck et al. (1982) daarentegen activeerden kennis over een onderwerp waarmee ze slechts ten dele vertrouwd waren. Tijdens het daaropvolgende informatieverwerkingsproces konden zij zich richten op nieuwe, relevante informatie; door deze informatie te integreren in het oorspronkelijke kennisbestand kon dit uitgebreid worden, resulterend in het beter onthouden van informatie die niet eerder geactiveerd werd.

Deze beschouwing kan verklaren waarom zo tegenstrijdige hypothesen zoals de selectieve-aandachtshypothese en de cognitieve set-point hypothese naast elkaar kunnen bestaan en waarom voor beide evidentie gevonden kon worden. Het onderzoek dat in Hoofdstuk II tot en met VI van dit proefschrift gepresenteerd wordt had tot doel deze verklaring nader te onderzoeken.

In Hoofdstuk II werd een pilotstudie gerapporteerd, die een tweeledig doel had. Allereerst werd het geconstrueerde materiaal getoetst. Nagegaan werd of de te bestuderen tekst informatie bevatte die correspondeerde met de door de proefpersonen geactiveerde voorkennis. Ten tweede werd onderzocht of mobilisatie een geschikte techniek is om voorkennis te activeren die of in een schema of in een minder stabiele kennisstructuur gerepresenteerd is.

De proefpersonen moesten een tekst bestuderen, getiteld "De gevolgen van het EG-visserijbeleid voor Crando Douro" (zie Appendix I). Deze tekst bevatte drie informatiecategorieën: (a) EG-informatie waarin de werking en de gevolgen van het door de EG gevoerde visserijbeleid werd beschreven, (b) kwekerij-informatie waarin een mogelijke oplossing geschetst werd voor de problemen die dit beleid met zich meebracht, en (c) toerisme-informatie waarin gerefereerd werd aan de natuurlijke schoonheid van het dorpje Crando Douro ("couleur locale") en zijn eventuele toeristische mogelijkheden. 
Voorafgaande aan het bestuderen van de tekst mobiliseerde de helft van de proefpersonen mondeling alle kennis die ze bezaten omtrent het door de EG gevoerde visserijbeleid en zijn gevolgen, een thema dat tijdens het afnemen van de experimenten in het middelpunt van de belangstelling stond; deze experimentele conditie werd de EG-groep genoemd. Verondersteld werd dat de specifieke kennis die door de EG-groep werd geactiveerd beperkt en nog onstabiel was. De voorkennis van de EG-groep werd geacht overeen stemmen met de EG-informatie in de tekst. Een tweede groep, de toerismegroep, werd gevraagd mondeling algemene factoren te mobiliseren die voor een touroperator van belang zijn om te bepalen of een bepaalde locatie geschikt zou zijn als nieuwe vakantiebestemming. Aangenomen werd dat de algemene kennis van de toerismegroep zich tot een schema ontwikkeld had door vele ervaringen op dit gebied. Verondersteld werd dat de slots van dit toerismeschema gevuld zouden kunnen worden met toerisme-informatie in de tekst. Om na te gaan of de tekst inderdaad informatie bevatte die overeenstemde met de geactiveerde voorkennis van de twee groepen, moesten de proefpersonen tijdens het bestuderen van de tekst alle informatiedelen markeren die relevant waren ten opzichte van hun instructie. Nadat elke zin van de tekst op deze manier één keer bestudeerd was, volgde een afleidende taak. Tenslotte moesten de proefpersonen mondeling alles rapporteren wat ze zich konden herinneren van de tekst.

De voornaamste conclusies die deze pilotstudie opleverde waren dat (a) de EG-informatie in de tekst inderdaad correspondeerde met de voorkennis van de EG-groep, (b) de toerisme-informatie overeenstemde met de voorkennis van de toerismegroep, en (c) mobilisatie een geschikte techniek leek te zijn om kennis te activeren, waardoor óf een selectieproces óf een expansieproces opgeroepen wordt.

Zowel in de selectieve-aandachtshypothese als in de cognitieve set-point hypothese speelt allocatie van studietijd een belangrijke rol. Het activeren van voorkennis zou bepalend zijn voor de wijze van allocatie van studietijd tijdens daaropvolgende tekstverwerking. Uit de twee hypothesen kunnen echter verschillende voorspellingen afgeleid worden met betrekking tot de informatiecategorie waaraan de meeste studietijd gewijd wordt. Teneinde de voorspellingen te toetsen van de twee hypothesen, die in dit proefschrift centraal staan, dienen dus zowel de recallpatronen als de allocatiepatronen van studietijd geregistreerd te worden. In Hoofdstuk III werd aan deze eisen voldaan. In een drietal experimenten werden de voorspellingen van de twee hypotheses getoetst.

In Experiment 1 was de totale hoeveelheid tijd, beschikbaar voor de bestudering van de tekst gefixeerd $\left(8^{\prime} 30^{\prime \prime}\right)$ maar deze tijd kon echter vrij verdeeld worden over de verschillende delen van die tekst. Voordat de tekst op een 
computerscherm bestudeerd werd, activeerde de EG-groep kennis met betrekking tot het EG-visserijbeleid; de toerismegroep activeerde kennis omtrent wat belangrijk is voor toerisme; een derde groep, de controlegroep, mobiliseerde kennis over een ongerelateerd onderwerp. Voorspeld werd dat de EGgroep de predicties van de cognitieve set-point hypothese zou reflecteren; de toerismegroep daarentegen zou studietijd- en recallpatronen vertonen die in overeenstemming zouden zijn met de selectieve-aandachtshypothese.

Verondersteld werd dat de EG-groep weinig tijd zou besteden aan EGinformatie aangezien zij deze informatie al gedeeltelijk verwerkt had tijdens de mobilisatiefase, waardoor minder tijd nodig zou zijn om de set-point voor die informatie te bereiken. Ook werd voorspeld dat de EG-groep meer tijd zou besteden aan nieuwe, relevante kwekerij-informatie aangezien zij, vergeleken met de controlegroep, hiervoor meer tijd zou hebben. De recallpatronen zouden in overeenstemming zijn met de allocatiepatronen van studietijd. Verwacht werd daarentegen dat de toerismegroep zich selectief zou richten op informatie in de tekst die relevant was voor haar "perspectief". Voorspeld werd dat de toerismegroep, vergeleken met de andere groepen, meer tijd zou besteden aan de verwerking van toerisme-informatie en daardoor deze informatie beter zou onthouden.

De door de EG-groep getoonde patronen van recall en allocatie van studietijd, vergeleken met die van de controlegroep, bevestigden grotendeels de voorspellingen van de cognitieve set-point hypothese. De EG-informatie werd inderdaad langer bestudeerd door de controlegroep dan door de EGgroep, terwijl deze groep het merendeel van de beschikbare studietijd besteedde aan de nieuwe, relevante kwekerij-informatie. De gevonden recallpatronen kwamen overeen met deze verschillen in informatieverwerking. De twee groepen verschilden niet in het onthouden van EG-informatie; de EGgroep behaalde echter hogere scores voor kwekerij-informatie aangezien deze groep, ten opzichte van de controlegroep, voldoende tijd had om de cognitieve set-point voor deze informatiecategorie te bereiken. Het verwachte verschil in totale recallscore bleek echter niet significant te zijn.

De toerismegroep overtrof, zoals verwacht, zowel de EG-groep als de controlegroep in het onthouden van toerisme-informatie. Deze score overschreed de recallscores van de toerismegroep voor elk van de resterende informatiecategorieën, die alle belangrijker waren in de tekst zelf. Het merendeel van de beschikbare studietijd werd an toerisme-informatie besteed. Aangezien de toerismegroep slechts $69 \%$ van de totale studietijd benutte, lijkt het niet vergezocht te concluderen dat deze groep zich selectief richtte op relevante informatie, waarbij alle andere informatiecategorieèn grotendeels genegeerd werden.

In Experiment 2 van Hoofdstuk III waren de proefpersonen volledig vrij in de allocatie van bestuderingstijd. De cognitieve set-point hypothese voor- 
spelde geen recallverschillen tussen de EG-groep en de controlegroep. De EGgroep zou echter minder tijd benodigen om de set-point voor EG-informatie te bereiken dan de controlegroep, waardoor de EG-groep in totaal minder tijd nodig zou hebben voor de verwerking van de tekst. De toerismegroep zou zich richten op informatie die relevant was ten opzichte van de geactiveerde kennis. Hierdoor zou deze groep meer tijd besteden aan toerisme-informatie en minder tijd aan de overige informatie. Deze wijze van allocatie van studietijd zou leiden tot het beter onthouden van toerisme-informatie, maar tot in totaal lagere recallprestaties. De selectieve-aandachtshypothese voorspelde bovendien dat de toerismegroep in total minder tijd zou benodigen.

De EG-groep onthield in total evenveel van de tekst als de controlegroep, maar zij benodigde hiervoor minder tijd. Het verschil in studietijd was gelegen in de hoeveelheid tijd die aan de EG-informatie gewijd werd. De controlegroep besteedde hieraan significant meer tijd dan de EG-groep. Deze bevindingen impliceren dat de EG-groep minder tijd voor die categorie benodigde omdat ze tijdens de mobilisatiefase al in de gelegenheid was vergelijkbare informatie te verwerken. Tijdens de studiefase kon de EG-groep hierdoor eerder de set-point voor EG-informatie bereiken dan de controlegroep.

De patronen van recall en allocatie van studietijd, die de toerismegroep vertoonde in de volledig vrije conditie (Experiment 2) waren nagenoeg identiek met de in Experiment 1 geconstateerde patronen. Deze groep proefpersonen blonk wederom uit in het onthouden van toerisme-informatie, die langer verwerkt werd dan elk van de resterende informatiecategorieën.

In Experiment 3 van Hoofdstuk III werd elke zin van de tekst gedurende een vooraf vastgestelde tijd gepresenteerd. De cognitieve set-point hypothese voorspelde dat de EG-groep alleen in staat zou zijn te profiteren van haar verwerkingsvoordeel, voortvloeiend uit het mobiliseren van voorkennis, wanneer bestuderingstijd vrij gedistribueerd kon worden over de diverse informatiedelen van de tekst. In een gefixeerde conditie werden daarom geen verschillen verwacht tussen de EG-groep en de controlegroep. De hoeveelheid tijd die besteed werd aan informatie, corresponderend met de geactiveerde kennis kon niet gereduceerd worden door de EG-groep; evenmin kon zij meer tijd besteden aan nieuwe, relevante informatie. Het verwerkingsvoordeel van de EG-groep zou dus teniet gedaan worden daar alle informatiecategorieèn door de beide groepen even lang verwerkt zouden worden. Verwacht werd dat de toerismegroep zich weer zou richten op informatie die overeenstemde met de eerder geactiveerde kennis, in zoverre dit mogelijk zou zijn in deze gefixeerde conditie; dit zou wederom leiden tot hogere recallscores voor die informatie.

De totale recallprestaties van de EG-groep en de controlegroep verschilden inderdaad niet. De twee groepen vertoonden feitelijk identieke recallpatronen voor de vier informatiecategorieën. 
Het recallpatroon van de toerismegroep verschilde nauwelijks van de eerdere gevonden patronen. Klaarblijkelijk was de beschikbare studietijd voldoende om toerisme-informatie te selecteren en te verwerken, resulterend in het beter onthouden van die informatie. De EG-informatie, de kwekerijinformatie en de residu-informatie werden slecht onthouden ondanks het feit dat de zinnen van deze categorieën gedurende een bepaalde tijd werden gepresenteerd. Dit suggereert dat deze informatie niet geincorporeerd kon worden in het geactiveerde schema daar die informatie niet overeenstemde met slots van het schema.

De bevindingen van Hoofdstuk III bieden steun voor zowel de cognitieve set-point hypothese als de selectieve-aandachtshypothese. De EG-groep, die de voorspellingen van de cognitieve set-point hypothese weerspiegelde, trachtte klaarblijkelijk haar beperkte kennis uit te breiden door zich op nieuwe, relevante informatie te concentreren. Wanneer enkel locale studietijd vrij was (Experiment 1) resulteerde haar verwerkingsvoordeel, ontstaan tijdens het mobiliseren van voorkennis, in hogere recallscores dan de controlegroep. In een volledig vrije conditie (Experiment 2 ) leidde dit tot een reductie van de hoeveelheid studietijd om een totale recallscore te kunnen behalen, gelijk aan die van de controlegroep. Dit verwerkingsvoordeel werd teniet gedaan in een volledig gefixeerde conditie (Experiment 3 ). De resultaten van de toerismegroep ondersteunen de selectieve-aandachtshypothese. Zowel met betrekking tot allocatie van studietijd als voor recall overheerste de slotvullende toerisme-informatie in alle condities, hetgeen het optreden van selectieve aandacht impliceert. De werking van selectieve aandacht werd met name gedemonstreerd in Experiment 3. Ook in deze gefixeerde conditie werd de slotvullende toerisme-informatie het best onthouden, alhoewel de resterende informatiecategorieën, die elk belangrijker waren in de tekst zelf, gedurende een bepaalde tijd gepresenteerd werden. Het zogenaamde levelseffect (Britton et al., 1979; Hustinx, 1985; Meyer, 1975), hetgeen inhoudt dat belangrijke informatie in een tekst het best onthouden wordt, werd dus verworpen door de toerismegroepen.

Alhoewel de toerismegroepen niet in staat leken te zijn de invloed van het activeren van een schema te weerstaan, zouden alternatieve verklaringen kunnen bestaan voor hun in Hoofdstuk III beschreven resultaten. Tijdens de interpretatie van de bevindingen van deze groepen doemden namelijk twee problemen op; in Hoofdstuk IV en Hoofdstuk V werden enkele experimenten beschreven waarin getracht werd deze anomalieën op te lossen.

In Hoofdstuk IV werd de aanname getoetst dat de patronen van recall en allocatie van studietijd, vertoond door de toerismegroepen in Hoofdstuk. III het gevolg zouden kunnen zijn van een zwakke experimentele instructie. Teneinde de proefpersonen een leesdoel te verstrekken werd hen verteld dat een 
tekst belangrijke en minder belangrijke informatie bevat. Hen werd uitgelegd dat het onderzoek waarin zij participeerden tot doel had na te gaan of belangrijke informatie onderscheiden kan worden van onbelangrijke informatie tijdens het bestuderen van een tekst. Deze instructie zou een ongewenste invloed gehad kunnen hebben op de proefpersonen van de toerismegroepen; zij zouden "belangrijk" geïnterpreteerd kunnen hebben als "belangrijk met betrekking tot de eerder gemobiliseerde kennis". Deze proefpersonen zouden dus aangenomen kunnen hebben dat zij zich tijdens de studiefase moesten richten op informatie die correspondeerde met de geactiveerde voorkennis. Indien deze assumptie accuraat zou zijn, zouden de in Hoofdstuk III geconstateerde patronen van recall en allocatie van studietijd niet de selectieveaandachtshypothese ondersteunen; zij zouden slechts bevestigen dat de gehanteerde instructie een onbedoeld neveneffect had.

Drie experimenten werden beschreven die tot doel hadden te onderzoeken of het mobiliseren van een schema een selectieproces oproept tijdens het verwerken van een tekst of slechts een leesdoel verstrekt voor de te bestuderen tekst. Twee groepen proefpersonen namen aan elk experiment deel: (a) De expliciete instructiegroep die geïnstrueerd werd te trachten zoveel mogelijk van de tekst in zijn geheel te onthouden; en (b) de impliciete instructiegroep die de mogelijk ambigue instructie kreeg, zoals gehanteerd in de experimenten van Hoofdstuk III. Wanneer de expliciete instructiegroepen patronen van recall en allocatie van studietijd vertoonden die identiek zouden zijn aan die van de impliciete instructiegroepen, zouden de eerder gevonden effecten niet toegeschreven kunnen worden aan een gebrekkige instructie.

De gewijzigde instructie leek de expliciete instructiegroepen in bepaalde mate beinvloed te hebben. Wanneer de locale studietijd vrij was (Experiment 1 en Experiment 2) besteedden de expliciete instructiegroepen meer tijd aan informatiecategorieën die een hoge plaats innemen in de hiërarchische tekststructuur dan de impliciete instructiegroepen. Deze allocatiepatronen van studietijd leidden tot in totaal hogere recallscores, hetgeen toegeschreven kon worden aan het beter onthouden van EG-informatie en kwekerij-informatie. Zelfs in een volledig gefixeerde conditie (Experiment 3) werd dit recallpatroon gevonden. Echter, slotvullende toerisme-informatie werd in alle condities nog steeds het best onthouden. Aan deze informatie werd de meeste tijd besteed, waardoor de hoogste recallscore voor toerisme-informatie werd behaald.

Concluderend kan gesteld worden dat de expliciete instructiegroepen, ondanks de expliciete instructie om zoveel mogellijk te onthouden van de tekst in zijn geheel, grotendeels de eerder gevonden patronen van recall en allocatie van studietijd vertoonden: Selectie van slotvullende toerisme-informatie, resulterend in hogere recallscores voor die informatie dan voor elk van de resterende informatiecategorieën van de tekst. Deze patronen van recall en alloca- 
tie van studietijd demonstreren de sterke invloed van het activeren van een schema op het verwerken en onthouden van informatie.

In Hoofdstuk $V$ werd een tweede probleem nader onderzocht, dat zich voordeed tijdens het interpreteren van de resultaten van de tourismegroepen in Hoofdstuk III. De toerismegroep leek namelijk niet in dezelfde mate te profiteren van het activeren van voorkennis als de EG-groep. Verondersteld werd dat de toerismegroep, net als de EG-groep, een voorsprong zou hebben op de controlegroep als gevolg van voorkennisactivatie. De controlegroep behaalde echter in totaal hogere recallscores dan de toerismegroep. Aangenomen werd dat de werking van selectieve aandacht hiervoor verantwoordelijk was. De in totaal lagere recallscores van de toerismegroep zouden echter ook verklaard kunnen worden door de wijze waarop de originele tekst opgebouwd was. De tekst bestond uit drie delen, die elk tien zinnen en 22 proposities telden: De EG-informatie, de kwekerij-informatie en de toerisme-informatie. Geargumen-teerd kan echter worden dat de EG-informatie en de kwekerijinformatie in feite één categorie vormden. Alhoewel de EG-groep voorkennis activeerde die alleen met de EG-informatie correspondeerde, zou de kwekerijinformatie beschouwd kunnen worden als een logische voortzetting van die informatie. Hierdoor zou de tekst twee keer zoveell informatie bevatten die relevant was voor de EG-groep als voor de toerismegroep. De in totaal lagere recallscores van de toerismegroep zouden dus kunnen voortvloeien uit dit verschil in hoeveelheid relevante informatie in de tekst.

De originele tekst werd herschreven om deze assumptie te kunnen toetsen. In de nieuwe tekst (zie Appendix III) werd het aantal proposities uitgebreid die voor de toerismegroep relevant zouden zijn, waardoor de tekst evenveel relevante informatie bevatte voor de toerismegroep als voor de EG-groep onder de aanname dat de EG-informatie en de kwekerij-informatie door de EG-groep als één categorie gezien werden. De nieuwe tekst werd aangeboden in een conditie waarin bestuderingstijd volledig vrij was. Onder de veronderstelling dat niet een verschil in verwerkingsstrategie maar enkel het verschil in relevante informatie verantwoordelijk zou zijn voor de bevindingen van Hoofdstuk III, werd verwacht dat de EG-groep en de toerismegroep in dezelfde mate zouden profiteren van voorkennisactivatie. De controlegroep zou dus in total meer tijd benodigen om een totale recallscore te behalen die de scores van de EG-groep en de toerismegroep zou evenaren.

De totale recallscore van de toerismegroep was echter nog altijd lager dan die van de EG-groep en de controlegroep, ondanks de relatieve toename in het onthouden van toerisme-informatie. De beide laatstgenoemde groepen proefpersonen blonken uit in het onthouden van EG- en kwekerij-informatie, terwijl de toerismegroep de hoogste scores voor toerisme-informatie behaalde. De controlegroep benodigde, zoals verwacht, in total meer tijd voor de be- 
studering van de tekst dan zowel de EG-groep als de toerismegroep. Opvallend was dat de toerismegroep, net als de EG-groep en de controlegroep, de meeste tijd besteedde aan EG-en kwekerij-informatie; de recallscores voor die informatie waren echter nog steeds laag. De toerismegroep leek dus getracht te hebben de expliciete instructie te volgen om zoveel mogelijk van de totale tekst te onthouden, hetgeen weerspiegeld werd door haar allocatiepatroon van studietijd. De EG- en de kwekerij-informatie werden echter slecht onthouden, ondanks de grote hoeveelheid tijd die aan deze informatie werd besteed; dit kan waarschijnlijk geweten worden aan het feit dat deze informatie geen slots van het geactiveerde schema vulde.

Teneinde de generaliseerbaarheid van de cognitieve set-point hypothese te toetsen werd in Hoofdstuk VI een poging ondernomen om de bevindingen van Hoofdstuk. II en Hoofdstuk III, die steun boden voor deze hypothese, uit te breiden naar itemlijsten. Drie experimenten werden beschreven waarin beschikbare bestuderingstijd op dezelfde wijze gemanipuleerd werd als in Hoofdstuk III. De proefpersonen werd, in plaats van een tekst, een itemlijst aangeboden, bestaande uit 16 namen van Amerikaanse presidenten en 16 namen van Amerikaanse staten. Aan elk experiment namen drie groepen proefpersonen deel. De proefpersonen mobiliseerden óf zoveel mogelijk namen van Amerikaanse presidenten (presidentengroep) óf van Amerikaanse staten (statengroep) óf van componisten (controlegroep). Verwacht werd dat de twee experimentele groepen, de presidentengroep en de statengroep, zouden profiteren van het mobiliseren van relevante voorkennis. Afhankelijk van de manipulatie van studietijd zouden de faciliterende effecten, voortvloeiend uit voorkennisactivatie, gelegen zijn in de totale recallscores of in de totale hoeveelheid tijd die nodig zou zijn voor de bestudering van de lijst.

Wanneer de totale studietijd gefixeerd was die echter vrij verdeeld kon worden over de 32 items (Experiment 2) behaalden de experimentele groepen inderdaad hogere recallscores dan de controlegroep. Zowel de presidentengroep als de statengroep benodigden minder tijd om items van de gemobiliseerde categorie te verwerken, waardoor relatief meer verwerkingstijd resteerde voor de andere, niet-geactiveerde categorie. Hierdoor waren de experimentele groepen in staat om de cognitieve set-point te bereiken voor meer items van de niet-geactiveerde categorie dan de controlegroep, hetgeen leidde tot in totaal hogere recallscores.

Wanneer de proefpersonen daarentegen vrij waren in het alloceren van bestuderingstijd (Experiment 1) kon de controlegroep haar achterstand wegwerken door meer tijd te besteden aan het bestuderen van de lijst. Geen recallverschillen werden gevonden tussen de beide experimentele groepen en de controlegroep. De controlegroep benodigde in totaal wel aanzienlijk meer tijd om recallprestaties te kunnen leveren, vergelijkbaar met die van de experimentele groepen daar die groepen, als gevolg van het mobiliseren van voor- 
kennis, minder tijd benodigden om de set-point te bereiken voor items van de gemobiliseerde categorie.

De experimentele groepen behaalden ook hogere recallscores dan de controlegroep in een volledig gefixeerde conditie (Experiment 3 ), hetgeen in strijd was met de voorspellingen van de cognitieve set-point hypothese. Voorspeld werd dat de experimentele groepen alleen zouden kunnen profiteren van het mobiliseren van voorkennis wanneer de allocatie van studietijd beïnloed zou kunnen worden. Minder tijd zou nodig zijn voor het verwerken van gemobiliseerde informatie, waardoor meer tijd zou resteren voor nietgeactiveerde informatie. Dit verwerkingsvoordeel zou teniet gedaan worden in een gefixeerde conditie, waardoor geen recallverschillen zouden kunnen optreden. In dit experiment behaalden de experimentele groepen echter hogere recallscores dan de controlegroep, hetgeen toegeschreven kon worden aan het beter onthouden van items van de gemobiliseerde categorie. Dit wederom werd geweten aan spreidende activatie, die tijdens mobilisatie zou hebben plaatsgevonden. Spreidende activatie zou kunnen optreden van gemobiliseerde items naar items van de toegewezen categorie die niet gemobiliseerd werden, waardoor deze items toegankelijker werden. Bijvoorbeeld, het nietgemobiliseerde item "Johnson" zou spreidende activatie ontvangen kunnen hebben van het gemobiliseerde item "Kennedy". Wanneer spreidende activatie inderdaad optreedt tijdens het mobiliseren van items van een bepaalde categorie zou verwacht kunnen worden dat niet-gemobiliseerde items van diezelfde categorie ook zouden profiteren van voorkennisactivatie, zij het in mindere mate dan gemobiliseerde items van die categorie. Additionele analyses ondersteunden deze assumptie.

Concluderend kan gesteld worden dat de cognitieve set-point hypothese een verklaring kan bieden voor de verwerking van zowel gemobiliseerde als niet-gemobiliseerde items van de geactiveerde categorie, mits deze theorie uitgebreid wordt met de assumptie dat spreidende activatie kan optreden tijdens het mobiliseren van voorkennis. Opvallend is dat er geen effecten van spreidende activatie optraden bij het verwerken van teksten (Hoofdstuk III), alhoewel dit een essentiële rol speelt in het constructie-integratiemodel van tekstverwerking (Kintsch, 1988).

\section{DISCUSSIE EN CONCLUSIES}

De eerste conclusie die getrokken kan worden uit de gerapporteerde bevindingen is dat de resultaten aanzienlijke steun bieden voor de idee dat tijdens het verwerken van informatie een cognitieve standaard voor comprehensie gehanteerd wordt, waaraan afgemeten kan worden of voldoende tijd is besteed teneinde deze standaard te bereiken. Aangetoond werd dat het mobiliseren van voorkennis onder geschikte condities kan resulteren in een af- 
name van de hoeveelheid tijd die besteed wordt aan het bestuderen van vergelijkbare informatie tijdens daaropwolgende tekstverwerking. Meer studietijd werd daarentegen besteed aan informatie waarmee het kennisbestand uitgebreid zou kunnen worden. De verschillende allocatiepatronen van studietijd van de experimentele (EG-)groep versus de controlegroep in Hoofdstuk III zijn congruent met de idee dat informatieverwerking onderhevig is aan een cognitieve set-point, die op de gehele verwerkingscyclus, inclusief de mobilisatiefase, betrekking heeft. Deze conclusies worden algemeen bevestigd en verruimd door de resultaten van Hoofdstuk VI.

Een tweede conclusie die het gerapporteerde onderzoek opleverde is dat de twee hypothesen die in dit proefschrift onderzocht werden, de cognitieve setpoint hypothese en de selectieve-aandachtshypothese, elkaar niet per definitie hoeven uit te sluiten. De beide hypothesen zouden verschillende fasen kunnen reflecteren in het proces van kennisverwerving, zoals voorgesteld door Schmidt en Boshuizen (1992).

De cognitieve set-point hypothese zou van toepassing kunnen zijn wanneer voorkennis wordt geactiveerd, waarvan aangenomen kan worden dat deze beperkt en nog instabiel is. De aard van de geactiveerde kennis zou een expansieproces oproepen tijdens het eropvolgende informatieverwerkingsproces. Nieuwe, relevante informatie zou grondig bestudeerd worden teneinde het gelimiteerde kennisbestand te kunnen uitbreiden. Dit zou wederom leiden tot de door de cognitieve set-point hypothese voorspelde recallpatronen: Het beter onthouden van informatie die vooraf niet geactiveerd werd.

De selectieve-aandachtshypothese zou daarentegen van toepassing zijn wanneer kennis geactiveerd wordt, waarvan aangenomen kan worden dat deze zich tot een tamelijk stabiele kennisstructuur ontwikkeld heeft door vele ervaringen in een bepaald domein. Het activeren van zo'n schema zou een selectieproces oproepen tijdens het verwerken van een tekst. Informatie die zou corresponderen mel slots van het geactiveerde schema zou geselecteerd worden, hetgeen zou leiden tot het recallpatroon zoals voorspeld door de selectieve-aandachtshypothese: Het beter onthouden van informatie die correspondeert met de geactiveerde voorkennis.

Alhoewel de door de EG-groepen en toerismegroepen vertoonde patronen van recall en allocatie van studietijd in overeenstemming zijn met de eerder beschreven theorie van kennisverwerving, mag de lezer echter niet uit het oog verliezen dat we in dit proefschrift niet in staat zijn geweest directe evidentie te leveren voor de aard van de cognitieve structuren die in dit proces van kennisverwerving een voorname plaats innemen.

Een derde conclusie die afgeleid kan worden uit de gepresenteerde resultaten is dat tijdens de mobilisatiefase meer geactiveerd wordt dan de kennis die 
feitelijk geproduceerd werd door de proefpersonen. In het laatste experiment van Hoofdstuk VI werden faciliterende effecten van voorkennisactivatie geconstateerd in een volledig gefixeerdle conditie. De experimentele groepen overtroffen de controlegroep in het totaal aantal onthouden items, hetgeen toegeschreven kon worden aan het beter onthouden van items van de gemobiliseerde categorie. De hogere recallscores die gevonden werden in deze gefixeerde conditie werden geweten aan het optreden van spreidende activatie tijdens mobilisatie. Niet-gemobiliseerde items binnen de geactiveerde categorie zouden tijdens mobilisatie spreidende activatie kunnen ontvangen van gemobilliseerde items, waardoor deze niet-gemobiliseerde items toegankelijker zouden worden. Dit zou wederom verklaren waarom voor deze nietgemobiliseerde items hogere recallscores behaald konden worden. Additionele analyses ondersteunden deze assumptie.

De vraag of spreidende activatie ook optreedt bij het verwerken van teksten blijft onbeantwoord aangezien in Hoofdstuk III, waarin het studiemateriaal bestond uit een tekst, geen faciliterende effecten gevonden werden in een volledig gefixeerde conditie. Spreidende activatie speelt echter volgens het constructie-integratiemodel van Kintsch (1988) een belangrijke rol bij het verwerken van teksten.

Een vierde conclusie heeft betrekking op de generaliseerbaarheid van het generation-effect (McDaniel et al., 1990; Slamecka \& Graf 1978), dat een alternatieve verklaring zou kunnen bieden voor het recallpatroon dat in het derde experiment van Hoofdstuk VI gevonden werd. Het generation-effect betreft het fenomeen dat items die door een proefpersoon zelf gegenereerd worden beter onthouden worden dan items die de proefpersoon door een experimentator aangeboden wordt. Wanneer deze hypothese van toepassing zou zijn op het derde experiment in Hoofdstuk VI zou men verwachten dat niet alleen items van de gemobiliseerde categorie beter onthouden zouden worden dan items van de niet-gemobiliseerde categorie, maar - en dit is veel belangrijker dat ook gemobiliseerde items binnen een categorie beter onthouden worden dan niet-gemobiliseerde items van diezelfde categorie. Een additionele analyse toonde echter juist het tegendeel aan. De hogere recallscores van de experimentele groepen voor de gemobiliseerde categorie konden volledig worden toegeschreven aan het beter onthouden van niet-gegenereerde items binnen de geactiveerde categorie. Deze afwijkende resultaten zouden toegeschreven kunnen worden aan verschillen in experimentele manipulaties; de gerapporteerde bevindingen zouden echter de generaliseerbaarheid van het generation-effect limiteren tot het paradigma waarin items gegenereerd versus bestudeerd worden. 



\section{REFERENCES}

Alba, J.W., \& Hasher, L. (1983). Is memory schematic? Psychological Bulletin, 93, 203231.

Alba, J.W., Alexander, S.G., Hasher, L., \& Caniglia, K. (1981). Notes, comments, and new findings; The role of context in the encoding of information. Journal of Experimental Psychology: Human Learning and Memory, 7, 283-292.

Albrecht, J.E., \& O'Brien, E.J. (1991). Effects of centrality on retrieval of text-based concepts. Journal of Experimental Psychology: Learning, Memory, and Cognition, 17, 932-939.

Anderson, J.R. (1983). The Architecture of Cognition. Cambridge: Harvard University Press.

Anderson, J.R. (1987). Skill acquisition: Compilation of weak-method problem solutions. Psychological Review, 94, 192-210.

Anderson, J.R. (1990). Meaning-based knowledge representations. In Cognitive Psychology and its Implications. (pp. 112-145). New York: Freeman.

Anderson, R.C. (1977). The notion of schemata and the educational enterprise: General discussion of the conference. In R.C. Anderson, R.J. Spiro, \& W.E. Montague (Eds.), Schooling and the Acquisition of Knowledge. (pp. 415-431). Hillsdale, NJ: Erlbaum.

Anderson, R.C. (1978). Schema-directed processes in language comprehension. In A. Lesgold, J. Pellegrino, S. Fokkema, \& R. Glaser (Eds.), Cognitioe Psychology and Instruction. (pp. 67-82). New York: Plenum Press.

Anderson, R.C. (1982). Allocation of attention during reading. In A. Flammer, \& W. Kintsch (Eds.), Discourse Processing. (pp. 292-305). Amsterdam: North-Holland.

Anderson, R.C., \& Pearson, P.D. (1984). A schema-theoretic view of basic processes in reading comprehension. In P.D. Pearson (Ed.), Handbook of Reading Research. (pp. 255-291). New York: Longman. 
Anderson, R.C. Pichert, J.W. (1978). Recall of previously unrecallable information following a shift in perspective. Journal of Verbal Learning and Wethol Betavior, 17 . $1-12$.

Anderson, $\mathrm{R}, \mathrm{C}_{2}$, Picher, I.W. \& Shirey, L.L. (1983). Effects of the reader's schema at different point in time. Journal of Educational Psychology, 75, 271-278.

Anderson, $\bar{R} \bar{C}$, Reynolds, R.E., Schaller, D. \&. Goetz, E.T. (1977). Frameworks for comprehending discourse. American Educational Research foumal, 14, 367-381.

Andre, T. (1979). Does answering higher-level questions while reading facilitate productive learning? Retiew of Educational Research $49,280-318$.

Ausubel, D.P. (1960). The use of adwance organizers in the learning and retention of meaningful verbal material. Jourtal of Educational Psychology, 51, 267-272.

Ausubel, D.P. (1963). The Psychology of Meaning ful Verbal Leaming. New York. Grune and Stratton.

Ausubel, D.P. (1968). Educational Psychology: A Cognitive Vew. New York: Holt, Rinehart, and Winston,

Ausubel, D.P. \& Youssef, M. (1963). Role of discriminability in meaningtul parallel leaming. Journal of Educational Psychology, 54, 331-336.

Barsalou, L.W. (1991). Deriving categories to achieve goals. In G.H. Bower (Ed.), The Psychology of Learting and Motwation. Adrances in Research and Theory. Volume 27. (pp. 1-64). New York Academic Press.

Berman, J.S., Read, S.J, \& Kenny, D.A. (1983). Processing inconsistent social information. Joumal of Personality and Social Psychology, 45, 1211-1224.

Beveridge, M. \& Parkins, E. (1987). Visual representation in analogical problem solving. Memory \& Cognition, 15, 230-237.

Boshuizen, H.P.A. (1989). De Ontwikkeling van Medische Expertise IOn the Development of Medical Expertise]. Unpublished dissertation. Maastricht: University of Limburg.

Bower, G.H., Black, J.B., \& Tumer, T.J. (1979). Scripts in memory for text. Cognitive Psychology, 11, 177-220.

Bransford, I.D. (1979). Human Cognition. Belmont, CA: Wadsworth.

Bransford, J.D., \& Franks, J.. (1976). Toward a framework for understanding learning. In G.H. Bower (Ed.), The Psychology of Learning and Motivation, Volume 10. (pp. 94-127). New York: Academic Press.

Bransford, J.D., \& Johnson, M.K. (1972). Contextual prerequisites for understanding: Some investigations of comprehension and recall. Journal of Verbal Learning and Verbal Behavior, 11, 717-726.

Brewer, W.F. \& Nakamura, G.V. (1984). The nature and functions of schemas. In R.S. Wyer, Jr., \& T.K. Srull (Eds.), Hawdbook of Social Cognition. (pp. 119-160). Hillsdale, NJ: Erlbaum.

Britton, B.K., Meyer, B.J.F., Simpson, R., Holdredge, T.S., \& Curry, C. (1979). Effects of the organization of text on memory: Tests of two implications of a selective attention hypothesis. Journal of Experimental PSychology: Human Learning and Memory, 5, 496-506.

Chi, M.T.H., Feltovich, P.J., \& Glaser, R. (1981). Categorization and representation of physics problems by experts and novices. Cognitive Science, 5, 121-152. 
Chiesi, H.L., Spilich, G.J., \& Voss, J.F. (1979). Acquisition of dornain-related information in relation to high and low domain knowledge. Journal of Verbal Learning and Verbal Behavior, 18, 257-273.

Cohen, C.E. (1981). Person categories and social perception: Testing some boundaries of the processing effects of prior knowledge. Journal of Personality and Social Psychology, 40, 441-452.

Corkill, A.J., Glover, J.A., Bruning, R.H., \& Krug, D. (1988). Advance organizers: Retrieval context hypotheses. Journal of Educational Psychology, 80, 304-311.

Dellarosa, O. (1986). A computer simulation of children's arithmetic word problem solving. Behavior Research Methods, Instruments, \& Computers, 18, 147-154.

Dooling, D.J., \& Lachman, R. (1971). Effects of comprehension on retention of prose. Journal of Experimental Psychology, 88, 216-222.

Figler, M.H., Weinstein, A.R., Sollers III, J.J., \& Devan, B.D. (1992). Pleasure travel (tourist) motivation: A factor analytic approach. Bulletin of the Psychonomic Society, 30, 113-116.

Gagné, R.M. (1974). Essentials of Learning for Instruction. Hinsdale, IL: Dryden Press.

Gardner, E.T., \& Schumacher, G.M. (1977). Effects of contextual organization on prose retention. Journal of Educational Psychology, 69, 146-151.

Gick, M.L., \& Holyoak, K.J. (1980). Analogical problem solving. Cognitive Psychology, 12, 306-355.

Gick, M.L., \& Holyoak, K.J. (1983). Schema induction and analogical transfer. Cognitive Psychology. 15, 1-38.

Glover, J.A., Ronning, R.R., \& Bruning, R.H. (1990). Cognitive Psychology for Teachers. (pp. 102-106). New York: MacMillan.

Goetz, E.T., Schallert, D.L., Reynolds, R.E., \& Radin, D.I. (1983). Reading in perspective: What real cops and pretend burglars look for in a story. Journal of Educational PSychology, 75, 500-510.

Grabe, M.D. (1979). Reader imposed structure and prose retention. Contemporary Educational Psychology, 4, 162-171.

Graesser, A.C., Gordon, S.E., \& Sawyer, J.D. (1979). Recognition memory for typical and atypical actions in scripted activities; Tests of a script pointer+tag hypothesis. Journal of Verbal Learning and Verbal Behawior, 18, 319-332.

Grimes, J.E. (1975). The Thread of Discourse. The Hague: Mouton.

Haviland, S., \& Clark, H.H. (1974). What's new? Acquiring new information as a process in comprehension. Journal of Verbal Learning and Verbal Behwoior, 13, 512-521.

Hirshman, E, \& Bjork, R.A. (1988). The generation effect: Support for a two-factor theory. Journal of Experimental Psychology: Learming, Monory, and Cognition, 14, 484494.

Hobus, P.P.M., Hofstra, M.L., Boshuizen, H.P.A., \& Schmidt, H.G. (1988). De context van de klacht als diagnosticum [Complaint context as diagnosticum]. Huisarts en Wetenschap, 31, 261-267.

Holyoak, K.J. (1990). Problem solving. In D.N. Osherson, \& E.E. Smith (Eds.), Thinking. An Invitation to Cognitive Science, Volume 3. (pp. 117-147). Cambridge: M.I.T.

Hout Wolters, B.H.A.M. van (1986). Markeren van Kerngedeelten in Studieteksten [Marking Central Parts in Study Texts]. Lisse: Swets \& Zeitlinger. 
Hustinx, L.G.M.M. (1985). Selectieve Verwerking van Informatie uit Tekst [Selective text processing]. Unpublished manuscript. Nijmegen: Katholieke Universiteit Nijmegen.

Johnson, W., \& Kieras, D. (1983). Representation-saving effects of prior knowledge in memory for simple technical prose. Memory \& Cognition, 11, 456-466.

Kardash, C.M., Royer, J.M., \& Greene, B.A. (1988). Effects of schemata on both encoding and retrieval from prose. Journal of Educational Psychology, 80, 324-329.

Keenan, J.M., \& Kintsch, W. (1974). Memory for information inferred during reading. In W. Kintsch (Ed.), The Representation of Meaning in Memory. (pp. 153-166). Hillsdale, NJ: Erlbaum.

Kintsch, W. (1980). Learning from text; Levels of comprehension, or: Why anyone would read a story anyway. Poetics, 9, 87-98.

Kintsch, W. (1988). The role of knowledge in discourse comprehension: A construction-integration model. Psychological Review, 95, 163-182.

Koedinger, K.R., \& Anderson, J.R. (1990). Abstract planning and perceptual chunks: Elements of expertise in geometry. Cognitive Science, 14, 511-550.

Lambiotte, J.G., \& Dansereau, D.F. (1990, April). Knowledge Maps and Prior Knowledge: Interactitze Effects on Recall. Paper presented at the annual meeting of the American Educational Research Association. Boston, MA.

Lee-Sammons, W.H., \& Whitney, P. (1991). Reading perspectives and memory for text: An individual differences analysis. Journal of Experimental Psychology: Learning, Memory, and Cognition, 17, 1074-1081.

Lintsen, T. (1984). Het Perspectief op de Tekst [Perspective on Text]. Unpublished manuscript. Nijmegen: Katholieke Universiteit Nijmegen.

Machiels-Bongaerts, M. (1993). Mobilizing Prior Knowledge in Text Processing. Unpublished dissertation. Maastricht: University of Limburg.

Machiels-Bongaerts, M., \& Boshuizen, H.P.A. (1989). Effectiever Leren door het Mobiliseren van Voorkennis: Alleen een Kwestie van Tijd? [Learning More Effectively as a Result of Mobilizing Prior Knowledge: Just a Matter of Time?] Onderzoek van Onderwijs, 40. Maastricht: University of Limburg.

Machiels-Bongaerts, M., Schmidt, H.G., \& Boshuizen, H.P.A. (1990, April). The Effect of Prior Knowledge Activation on Text Recall: An Investigation of Two Conflicting Hypotheses. Paper presented at the annual meeting of the American Educational Research Association. Boston, MA.

Machiels-Bongaerts, M., Schmidt, H.G., \& Boshuizen, H.P.A. (1991, April). The Effects of Prior Knowledge Activation on Free Recall and Study Time Allocation. Paper presented at the annual meeting of the American Educational Research Association. Chicago, IL.

Machiels-Bongaerts, M., Schmidt, H.G., \& Boshuizen, H.P.A. (1993, April). Cognitive Set-Points in Text Processing: Studies of Free Recall and Allocation of Study Time. Paper presented at the annual meeting of the American Educational Research Association. Atlanta, GA.

Machiels-Bongaerts, M., Schmidt, H.G., \& Boshuizen, H.P.A. (1993). Effects of mobilizing prior knowledge on information processing: Studies of free recall and allocation of study time. British Journal of Psychology, 4, 481-498. 
Machiels-Bongaerts, M., Schmidt, H.G., \& Boshuizen, H.P.A. (submitted). Selective Attention versus Cognitive Set-Point Hypothesis in Text Processing: Studies on Free Recall and Allocation of Study Time.

Mayer, R.E. (1976). Some conditions of meaningful learning for computer programming: Advance organizers and subject control of frame order. Journal of Educational Psychology, 68, 143-150.

Mayer, R.E. (1979). Can advance organizers influence meaningful learning? Review of Educational Research, 49, 371-383.

Mayer, R.E. (1980). Elaboration techniques that increase the meaningfulness of technical text: An experimental test of the learning strategy hypothesis. Journal of Educational Psychology, 72, 770-784.

Mayer, R.E. (1985). Structural analysis of science prose: Can we increase problemsolving performance? In B.K. Britton, \& J.B. Black, (Eds.), Understanding Expository Text. (pp. 65-87). Hillsdale, NJ: Erlbaum.

Mayer, R.E., \& Bromage, B.K. (1980). Different recall protocols for technical texts due to advance organizers. Journal of Educational Psychology, 72, 209-225.

McDaniel, M.A., Riegler, G.L., \& Waddill, P.J. (1990). Generation effects in free recall: Further support for a three-factor theory. Journal of Experimental Psychology: Learning, Memory, and Cognition, 16, 789-798.

Meyer, B.J.F. (1975). The Organization of Prose and its Effects on Memory. Amsterdam: North-Holland.

Meyer, B.J.F., Brandt, D., \& Bluth, G.J. (1980). Use of top-level structure in text: Key for reading comprehension in ninth-grade students. Reading Research Quarterly, $16,72-103$.

Meyer, B.J.F., \& McConkie, G.W. (1973). What is recalled after hearing a passage? Journal of Educational Psychology, 65, 109-117.

Meyer, B.J.F., \& Rice, G.E. (1984). The structure of text. In P.D. Pearson (Ed.), Handbook of Reading Research. (pp. 319-351). New York: Longman.

Minsky, M. (1975). A framework for representing knowledge. In P.M. Winston (Ed.), The Psychology of Computer Vision. (pp. 211-277). New York: MoGraw-Hill.

Nairne, J.S., \& Widner, R.L. (1987). Generation effects with nonwords: The role of test appropriateness. Journal of Experimental Psychology: Learning. Memory, and Cognition, $13,164-171$.

Niederhauser, D., \& Reynolds, R.E. (1991, April). The Influence of Metaphor on Prose Learning: Reconstructive versus Processing Explanations. Paper presented at the annual meeting of the American Educational Research Association. Chicago, IL.

Norman, D.A., Rumelhart, D.E., \& the LNR Research Group (1975). Explorations in Cognition. San Francisco: Freeman.

Nungester, R.J., \& Duchastel, P.C. (1982). Testing versus review: Effects on retention. Journal of Educational Psychology, 74, 18-22.

Paris, S.G. (1988). Models and metaphors of learning strategies. In C.E. Weinstein, E.T. Goetz \& P.A. Alexander (Eds.), Learning and Study Strategies. (pp. 299-321). New York: Academic Press.

Peeck, J. (1982). Effects of mobilization of prior knowledge on free recall. Journal of Experimental Psychology: Learning, Mernory, and Cognition, 8, 608-612. 
Peeck, J., Van den Bosch, A.B., \& Kreupeling, W.J. (1982). The effect of mobilizing prior knowledge on learning from text. Journal of Educational Psychology, 74,771777.

Perfetto, G.A., Bransford, J.D., Franks, J.J. (1983). Constraints on access in problem solving context. Memory $\mathcal{E}$ Cognition, 11, 24-31.

Pezdek, K, Whetstone, T., Reynolds, K., Askari, N., \& Dougherty, T. (1989). Memory for real-world scenes: The role of consistencey with schema expectation. Joumal of Experimental Psychology: Leaming, Memory, and Cognition, 15, 587-595.

Pichert, J.W., \& Anderson, R.C. (1977). Taking different perspectives on a story. Journal of Educational Psychology, 69, 309-315.

Rabinowitz J.C., \& Craik, F.I.M. (1986). Specific enhancement effects associated with word generation. Journal of Memory and Language, 25, 226-237.

Raney, G.E. (1993). Monitoring changes in cognitive load during reading: An eventrelated brain potential and reaction time analysis. Journal of Experimental Psycholosy: Learning, Memory, and Cognition, 19, 51-69.

Reynolds, R.E., \& Anderson, R.C. (1982). Influence of questions on the allocation of attention during reading. Journal of Educational Psychology, 74, 623-632.

Reynolds, R.E., \& Shirey, L.L. (1988). The role of attention in studying and learning. In C.E. Weinstein, E.T. Goetz, \& P.A. Alexander (Eds.), Learning and Study Strategies. (pp. 77-100). New York: Academic Press.

Reynolds, R.E., Standiford, S.N., \& Anderson, R.C. (1979). Distribution of reading time when questions are asked about a restricted category of text information. Journal of Educational Psychology, 71, 183-190.

Rickards, J.P., \& Denning, P.R. (1979). Depressive effects of underlining and adjunct questions on children's recall of text. Instructional Science, 8, 81-90.

Robertson, W.C. (1990). Detection of cognitive structure with protocol data: Predicting performance on physics transfer problems. Cognitive Science, 14, 253-280.

Rothkopf, E.Z., \& Billington, M.J. (1979). Goal-guided learning from text: Inferring a descriptive processing model from inspection times and eye movements. Journal of Educational Psychology, 71,310-327.

Rumelhart, D.E., \& Norman, D.A. (1978). Accretion, tuning, and restructuring: Three modes of learning. In J.W. Cotton, \& R.L. Klatzky (Eds.), Semantic Factors in Cognition. (pp. 37-53). Hillsdale, NJ: Erlbaum.

Rumelhart, D.E., \& Ortony, A. (1977). The representation of knowledge in memory. In R.C. Anderson, R.J. Spiro, \& W.E. Montague (Eds.), Schooling and the Acquisition of Knowoledge. (pp. 99-135). Hillsdale, NJ: Erlbaum.

Sanford, A.J., \& Garrod, S. (1981). Understanding Written Language. New York: Wiley.

Schank, R.C., \& Abelson, R.P. (1977). Scripts, Plans, Goals and Understanding. Hillsdale, NJ: Erlbaum.

Schmidt, H.G. (1982a). Activatie van Voorkennis, Intrinsieke Motivatie en de Verwerking tant Tekst [Prior Knowledge Activation, Intrinsic Motivation and Text Processing]. Apeldoorn: Van Walraven.

Schmidt, H.G. (1982b). Activation and restructuring of prior knowledge and their effects on text processing. In A. Flammer, \& W. Kintsch (Eds.), Discourse Processing. (pp. 325-338). Amsterdam: North-Holland. 
Schmidt, H.G. (1983a). Activatie van voorkennis en een "verschuivings" effect bij het onthouden van tekst [Prior knowledge activation and a "shift" in text recall]. Tijdschrift voor Taal-en Tekstwetenschap, 3, 84-93.

Schmidt, H.G. (1983b). Activatie van Voorkennis en Tekstwerwerking [Prior Knowledge Activation and Text Processing]. Request for funding submitted to the Institute for Educational Research in the Netherlands (SVO).

Schmidt, H.G. (1984). Activatie van voorkennis en tekstverwerking [Prior knowledge activation and text processing]. Nederlands Tijdschrift voor de PSychologie, 39, 335347.

Schmidt, H.G., \& Boshuizen, H.P.A. (1992). Encapsulation of biomedical knowledge. In D.A. Evans, \& V.L. Patel (Eds.), Advanced Models of Cognition for Medical Trainting and Practice. Berlin: Springer.

Schmidt, H.G., \& Gijselaers, W.H. (1990, April). Causal Modelling of Problem-based Learning. Paper presented at the annual meeting of the American Educational Research Association. Boston, MA.

Schraw, G., \& Wade, S. (1991, April). Selective Learning Strategies for Relewant and Important Text Information. Paper presented at the annual meeting of the American Educational Research Association. Chicago, iL.

Shuell, TJ. (1990). Phases of meaningful learning. Review of Educational Research, 60, 531-547.

Simons, P.R.J. (1984). Instructing with analogies. Journal of Educational Psychology, 76 , 513-527.

Slamecka, N.J., \& Graf, P. (1978). The generation effect: Delineation of a phenomenon. Journal of Experimental Psychology: Human Learning and Memory, 4, 592604.

Spilich, G.J., Vesonder, G.T., Chiesi, H.L., \& Voss, J.F. (1979). Text processing of domain-related information for individuals with high and low domain knowledge. Journal of Verbal Learning and Verbal Behavior, 18, 275-290.

Thorndyke, P.W. (1977). Cognitive structures in comprehension and memory of narrative discourse. Cognitive Psychology, 9, 77-110.

Vonk, W. (1986, September). Readers Perspective and Selective Processing. Paper presented at the Conference les Text et leur Traitement. Poitier, France.

Vosniadou, S., \& Brewer, W.F. (1987). Theories of knowledge restructuring in development. Review of Educational Research, 57, 51-67.

Vosniadou, S., \& Schommer, M. (1988). Explanatory analogies can help children acquire information from expository text. Journal of Educational Psychology, 80, 524536 .

Voss, J.F., Blais, J., Means, M.L., Greene, T.R., \& Aswesh, E. (1986). Informal reasoning and subject matter knowledge in the solving of economics problems by naive and novice individuals. Cognition and Instruction, 3, 269-302.

Voss, J.F., Tyler, S.W., \& Bisanz, G.L. (1982). Prose comprehension and memory. In C.R. Puff (Ed.), Handbook of Research Methods in Human Memory and Cognition. (pp. 349-393). New York: Academic Press.

Willems, J.M.H.M. (1987). Studietaken als Instructiemiddel [Study tasks as Instructional Aid]. Unpublished dissertation. Nijmegen: Katholieke Universiteit Nijmegen. 


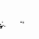




\section{THE CONSEQUENCES OF THE EEC FISHERY POLICIES FOR CRANDO DOURO ${ }^{1}$}

Since 1986, the EEC fishery policies are also in force for Portugal, which has led to an unemployment rise in many small fishing-villages. Due to the fish catch limitations the income dropped, which increased unemployment.

This holds, for instance, for Crando Douro with less than 14,000 inhabitants, located at the mouth of the river Douro; since many generations, this village is largely dependent on fishing. It is not only because of its mild climate - the average temperature in the summer amounts to 25 degrees and in the winter to 16 degrees - that this picturesque village is suited for this trade, but due to lack of education there practically is no alternative profession for the inhabitants.

The unemployment has enormously increased as a result of the EEC meatsurements. In 1986, the total amount of catch allowed for Crando Douro added up to 60,000 tons only, forcing the fishing-industries to cut back their concerns and to fire a large number of fishermen.

There are practically no prospects for the population since there is hardly any industrialization in the environment.

But although the existing situation offers little perspective, the inhabitants of Crando Douro are too dignified to accept their faith. It does not suit their mentality to live on unemployment benefits and to spend their gained spare time taking walks in the forest or lying on the beach.

As a consequence of the increase in unemployment, people are already inclined to move to the city, which will irrevocably lead to an ageing of villages like

1 The study texts applied in the experiments described in this dissertation could be constructed thanks to information provided by the Dutch Ministry of Agriculture and Fisheries and by the Portuguese Embassy, both situated in The Hague, the Netherlands; furthermore, we would like to thank Jeannet Kalk for correcting the translation of the original Dutch text. 
Crando Douro. Especially youngsters are ready to move to the big city. In the romantic Crando Douro only old people and women stay behind, still mastering the old crafts of producing the well-known pottery and needle-point works.

Although the EEC fishery policies have caused many problems, it is evident even for the inhabitants of Crando Douro that regulation by a co-ordinate institution was necessary. In this way overfishing can be avoided; in other words, the extent of the fish population diminishes due to a too intensive catch, which could lead to many fishing-industries going bankrupt.

The information above shows that the EEC measurements do not solely serve to preserve the extent of the fish population, but also to protect the fisheryindustries of the EEC nations. For instance, fishing-industries of non-members are no longer allowed to fish within a 200-miles zone, which should reduce the competition drastically.

Since 1977 , one of the EEC's tasks is to give a definite decree of the allowed amount of catch and to distribute these so-called "quota" over the member states. These quota or Total Allowed Catch, in short $\mathrm{TAC}_{x}$ are minutely specified by the EEC regarding fish breeds and catch areas, and always apply to restricted fishing seasons. Based on its research, the International Council for the Exploration of the Sea gives the EEC recommendations with respect to the tolerable maximum fish quota.

The various EEC guide-lines and regulations are supervized by the General Inspection Service of the Ministry of Agriculture and Fisheries. The General Inspection Service severely penalizes illegal fishing; in other words, to continue fishing even if the national quotum, accorded by the EEC, has already been caught. The illegally caught fish is confiscated and the fishermen warned, besides being penalized severely, that a repetition of this offence will lead to an arrest.

The question remains whether the fishermen of Crando Douro and the surrounding area, who have already been confronted with the measurements of the General Inspection Service, will obey the EEC regulations in the future. They want to support themselves even in this hopeless situation.

There are two potential solutions for Crando Douro's problems.

The first solution refers to initiate industrialization. In the near future, the Portuguese government tries to attract a number of foreign industries, which should settle at the west coast. The government of Portugal, the poorest country in Europe, wants to persuade these multinationals by means of the low value of the escudo and the huge market of cheap workmen.

The second solution is to start a fish-farm, for which the power-station at the Douro would be well-suited since the temperature of the cooling water is approximately eight degrees higher than the sea's. Fishes which are cold-blooded, as everybody may know, digest their food considerably faster in water with a tropical temperature, which increases growth substantially.

Under normal circumstances, it takes ten months for a fish to grow ten centers, whereas in warm water it takes them half that time. As a result of this dynamic increase in growth, the production, and thus sales, could almost be doubled while the costs would remain relatively low. 
In order to establish an optimal growth process, a constant water temperature and an ideal oxygen level are the most essential prerequisites. The temperature can be ruled to one degree exactly by mixing cooling water and regular water; the oxygen level can be controlled by means of simple equipment. An unnegligible side-effect of the constant water temperature is that the probability of disease germs to develop in the basins would be considerably reduced.

An example of this renewed method of breeding is located in Genk, Belgium, where this modern technique has been successfully applied since several months. Besides the well-known trouts and carps, especially tropical Tilapias are cultivated in the Belgian basins, which appear to be well-suited for this way of fish breeding. The Tilapia, which is also often referred to as "the fish of the future $^{\prime \prime}$, comes from Africa and is considered as an exclusive delicacy in the coastal region of the South of France.

Apart from the advantages which hold for the alternative solution described earlier as well as for the solution proposed by the government, a number of disadvantages can be pointed out which are of great importance.

With respect to the first disadvantage, which is the inadequate infrastructure, the state is willing to meet the multinationals halfway by approving an extension of the road-system. This financial contribution has been justified by the Portuguese government with the argumentation that this investment could in the long run be compensated for by reduction of social expenses, which would be the result of the employment created. At this very moment, however, this concession would affect the already weak financial position of Portugal.

Naturally, the inferior condition of the infrastructure is also an obstacle for the second approach of the unemployment problem in this area. However, in this case a cheaper solution could be passed, namely transport by water since the largest fish market is situated in France.

The tradition of fishing in Crando Douro would be preserved, thanks to this solution. The ancient character of this village with its market stalls, restaurants serving local dishes, pavements and "estalagens" (little inns) could be saved after all.

In the meantime, an extension of the future perspectives could be realized, while the centuries old traditions would not disappear. The people are faithful to tradition, which is shown by the fact that they hold on to the traditional bullfights in which the bull is not killed in the arena, contrary to the Spanish bullfights.

Another ponderous aspect concerns the environmental pollution that comes along with industrialization. If the chemical industries dumped their waste materials in the sea, this would form an increasing threat to the already diminishing fish stock.

Not only would the chance of a fish stock restoration decline, but the EEC fishery policies, causing so many problems in Crando Douro and the surrounding areas, would also be pointless. Water sports like swimming, deep-sea diving, surfing and sailing, for which the Portuguese coast is ideal, could no longer be practiced due to the pollution. 
In short, industrialization does not seem to be the right answer neither for Crando Douro nor for Portugal.

If the Portuguese government still chooses this allernative, the population of Crando Douro has an extra reason to strike up one of their elegies. The population's grief is expressed in the "saudades", songs so characteristic for this region and often heard in the small pubs in the harbour - there are no discotheques in Crando Douro.

Finally, they can take a pilgrimage to the neighbouring Fatima, where they can pray to God for a miracle in the medieval church with its unique quarrels. 
APPENDIX II

\section{HIERARCHICAL TEXT STRUCTURE 1}

The text "The consequences of the EEC fishery policies for Crando Douro" (see Appendix I) contained 57 sentences, consisting of 100 propositions. The EEC information, the fish-farm information and the tourism information were each described in ten sentences, which could be divided into 22 propositions. The residual information consisted of 27 (short) sentences, which contained 34 propositions.

Meyer's (1975) method was applied to establish the relations between propositions of a text. It should be pointed out that Meyer's system, based on Grimes' (1975) model to describe the organization of a text, is more refined than the one applied in the studies reported in this thesis. Like Schmidt (1982a), a less detailed classification was chosen to simplify the pratical application.

The mean level of the EEC information in the text hierarchy was 4.23 (ranging from level 2 to 6). The mean numbers of words, syllables, and characters per sentence were 27,52 , and 178 , respectively. ${ }^{1}$ Exactly the same notions apply to the fish-farm information. The mean level of the tourism information in the hierarchical text structure was 6.63 (ranging from level 3 to 11). The mean numbers of words, syllables, and characters for this information category were 28, 51, and 179 , respectively. The mean level of the residual information in the text hierarchy was 5.17. The sentences of this information category were shorter than those of the remaining categories; the mean number of words per sentences was 16 for the residual information.

The hierarchical structure of the text applied in the studies of Chapters II to IV is presented on the next page.

\footnotetext{
${ }^{1}$ It should be pointed out that these notions refer to the original text version.
} 


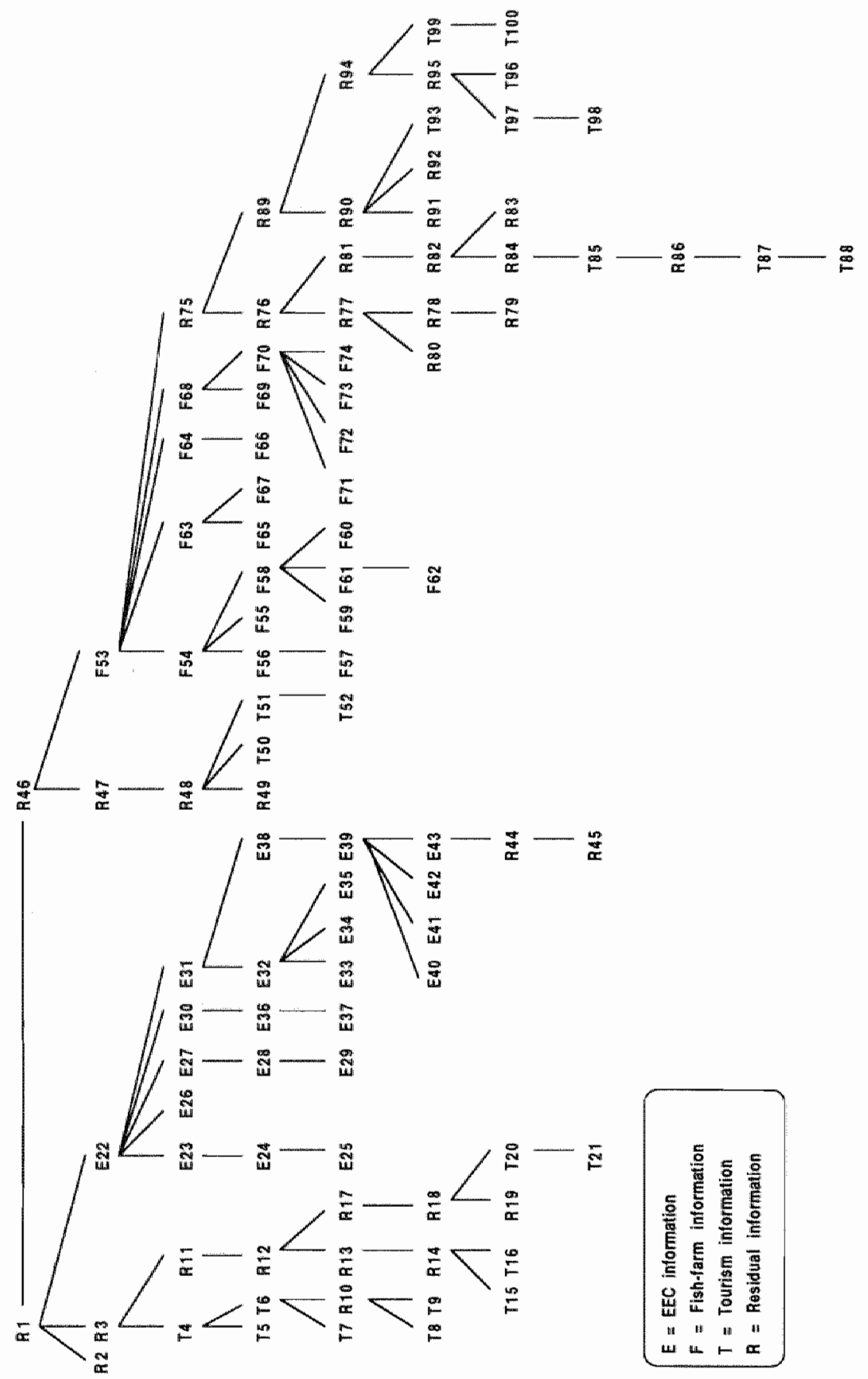




\section{CRANDO DOURO}

Crando Douro is the name of a village with less than 14,000 inhabitants, located at the mouth of the river Douro in south-west Portugal. Since many generations, this village is largely dependent on fishing since there is hardly any industrialization in this area. Furthermore, Crando Douro is suited for this trade because of its mild climate - the average temperature in the summer amounts to 25 degrees and in the winter to 16 degrees.

Since 1986, the EEC fishery policies are also in force for Portugal, which has caused many problems in Crando Douro. Due to the EEC measurements the income dropped, which increased unemployment in small fishing-villages like Crando Douro.

But although the existing situation offers little perspective, the inhabitants of Crando Douro are too dignified to accept their faith. It does not suit their mentaility to live on unemployment benefits and to spend their gained spare time taking walks in the forest or lying on the beach.

As a consequence of the increase in unemployment, people are already inclined to move to the city. Especially youngsters are ready to move to the big city, in which the environmental pollution is worse, the city center is less attractive, and the unemployment rate is at least as high. In the old-fashioned Crando Douro only old people and women will stay behind, still mastering the old crafts of producing the well-known pottery and needle-point works.

The people are faithful to tradition, which is shown by the fact that they hold on to the traditional bull-fights in which the bull is not killed in the arena, contrary to the Spanish bull-fights. The inhabitants of Crando Douro also hold on to the folkore dances, like the boltanella, which is demonstrated in the market-place during the summer. 
Although the EEC fishery policies have caused many problems, it is evident even for the inhabitants of Crando Douro that regulation by a co-ordinate institution was necessary. Overfishing can be avoided in this way; in other words, due to a too intensive catch, the extent of the fish population diminishes, which could lead to many fishing-industries going bankrupt.

The information above shows that the EEC measurements do not solely serve to preserve the extent of the fish population, but also to protect the fisheryindustries of the EEC nations. For instance, fishing-industries of non-members are no longer allowed to fish within a 200-miles zone, which should reduce the competition drastically.

Since 1977, one of the EEC's tasks is to give a definite decree of the allowed amount of catch and to distribute these so-called "quota" over the member states. These quota or Total Allowed Catch, in short TAC, are minutely specified by the EEC regarding fish breeds and catch areas, and always apply to restricted fishing seasons. Based on its research, the International Council for the Exploration of the Sea gives the EEC recommendations with respect to the tolerable maximum fish quota.

The various EEC guide-lines and regulations are supervized by the General Inspection Service of the Ministry of Agriculture and Fisheries. The General Inspection Service severely penalizes illegal fishing; in other words, to continue fishing even if the national quotum, accorded by the EEC, has already been caught. The illegally caught fish is confiscated and the fishermen warned, besides being penalized severely, that a repetition of this offence will lead to an arrest.

The question remains whether the fishermen of Crando Douro and the surrounding area, who have already been confronted with the measurements of the General Inspection Service, will obey the EEC regulations in the future. They want to support themselves even in this hopeless situation.

The EEC fishery policies and especially their consequences could stir up quite some commotion in Crando Douro. Normally, Crando Douro is a peaceful village, where one can relax on a bench at the banks of the Douro, enjoying the virgin forests. Climbing the Dolgi hill provides a panoramic view of the surroundings of Crando Douro, which is not dominated yet by huge hotels and apartments. Recently, a camping site is located next to the picturesque harbour, but it can only accommodate 100 tents and caravans; it is equiped with a playground, tennis and golf lanes and a swimming pool. The inhabitants of Crando Douro, however, prefer a swim in the sea water, which has a temperature of 20 degrees; from the center of Crando Douro, this takes a walk of ten minutes at the most.

There are two potential solutions for Crando Douro's problems.

The first solution refers to initiate industrialization. In the near future, the Portuguese government tries to attract a number of foreign industries, which should settle at the west coast. The government of Portugal, the poorest country in Europe, wants to persuade these multinationals by means of the low value of the escudo and the huge market of cheap workmen. 
The second solution is to start a fish-farm, for which the power-station at the Douro would be well-suited since the temperature of the cooling water is approximately eight degrees higher than the sea's. Fishes which are cold-blooded, as everybody may know, digest their food considerably faster in water with a tropical temperature, which increases growth substantially.

Under normal circumstances, it takes ten months for a fish to grow ten centers, whereas in warm water it takes them half that time. As a result of this $d y$ namic increase in growth, the production, and thus sales, could almost be doubled while the costs would remain relatively low.

In order to establish an optimal growth process, a constant water temperature and an ideal oxygen level are the most essential prerequisites. The temperature can be ruled to one degree exactly by mixing cooling water and regular water; the oxygen level can be controlled by means of simple equipment. An unnegligible side-effect of the constant water temperature is that the probability of disease germs to develop in the basins would be considerably reduced.

An example of this renewed method of breeding is located in Genk, Belgium, where this modern technique has been successfully applied since several months. Besides the well-known trouts and carps, especially tropical Tilapias are cultivated in the Belgian basins, which appear to be well-suited for this way of fish breeding. The Tilapia, which is also often referred to as "the fish of the future", comes from Africa and is considered as an exclusive delicacy in the coastal region of the South of France.

Apart from the advantages which hold for the alternative solution described earlier as well as for the solution proposed by the government, a number of disadvantages can be pointed out which are of great importance.

With respect to the first disadvantage, which is the inadequate infrastructure, the state is willing to meet the multinationals halfway by apporving an extension of the road-system. This financial contribution has been justified by the Portuguese government with the argumentation that this investment could in the long run be compensated for by reduction of social expenses, which would be the result of the employment created. At this very moment, however, this concession would affect the already weak financial position of Portugal.

Naturally, the inferior condition of the infrastructure is also an obstacle for the second approach of the unemployment problem in this area. However, in this case a cheaper solution could be passed, namely transport by water since the largest fish market is situated in France. The tradition of fishing in Crando Douro would be preserved, thanks to this solution. The ancient character of this village with its market stalls, pavements, "estalagens" (little inns) and romantic restaurants could be saved after all. In the cosy restaurants the menu is naturally dominated by all kinds of local dishes with fish; the guests are allowed to go into the kitchen in order to select their choices. This custom shows the hospitality of the inhabitants of Crando Douro, who don't have to fear this peek into the kitchen since the standards of hygiene are quite high.

Another ponderous aspect concerns the environmental poluution that comes along with industrialization. If the chemical industries dumped their 
waste materials in the sea, this would form an increasing threat to the already diminishing fish stock.

Not only would the chance of a fish stock restoration decline, but the EEC fishery policies, causing so many problems in Crando Douro and the surrounding areas, would also be pointless. Due to the pollution, water sports like swimming, deep-sea diving, surfing and sailing, for which the Portuguese coast is ideal, could no longer be practiced.

In short, industrialization does not seem to be the right answer neither for Crando Douro nor for Portugal.

If the Portuguese government still chooses this alternative, the population of Crando Douro has an extra reason to strike up one of their elegies. The population's grief is expressed in the "saudades", songs so characteristic for this region and often heard in the small pubs in the harbour - there are no discotheques in Crando Douro.

Finally, they can take a pilgrimage to the neighbouring Fatima, where they can pray to God for a miracle in the medieval church with its unique quarrels. 


\section{HIERARCHICAL TEXT STRUCTURE 2}

On the next page the proposition tree of the text "Crando Douro" is depicted (see Appendix III), which was applied in the study presented in Chapter V. Again, Meyer's (1975) method was used, albeit less refined, to describe the text's hierarchical structure. The most important information is placed on high levels in this tree, whereas less important information resides at lower levels in this structure.

The text consisted of 61 sentences, which could be divided into 115 propositions. The $\mathbb{E E C}$ information and the fish-farm information each contained ten sentences, divided into 22 propositions. The tourism information consisted of 20 sentences, which could be divided into 44 propositions. The residual information consisted of 21 short sentences, containing 27 propositions.

The EEC information's mean level in the text hierarchy was 5.64 (ranging from level 3 to level 8), The mean number of words, syllables, and characters per sentence were 27,52 and 178, respectively. Exactly the same notions apply to the fish-farm information. The mean level of the tourism information was 7.34 (ranging from level 1 to level 15). The mean number of words, syllables, and characters of this information category were 28,52 and 177 , respectively. The mean level of the residual information in the text's hierarchical structure was 5.04 (ranging from level 2 to level 9).

In the study presented in Chapter $V$, the EEC information and the fish-farm information were combined to the so-called "non-tourism information"; this information category equaled the tourism information in numbers of sentences and propositions (i.e., 20 sentences and 44 propositions).

1 It should be pointed out explicitely that these notions refer to the original Dutch wersion of the text. 


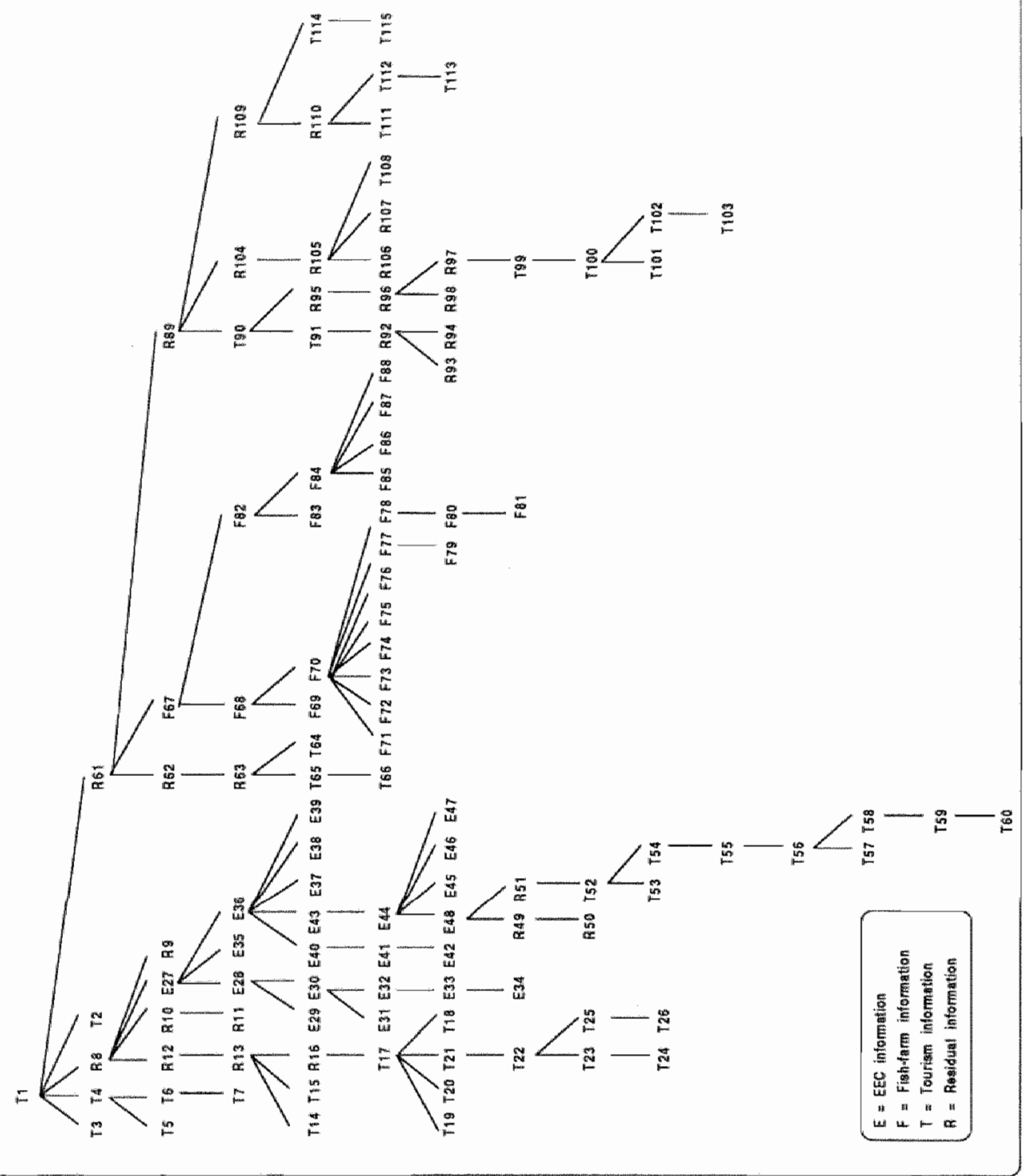




\section{CURRICULUM VITAE}

Maureen Machiels-Bongaerts was born on April 6, 1962 in Echt. She finished (Atheneum $\alpha$ ) at the Bisschoppelijk College Echt in 1980. She studied Dutch literature and linguistics at the University of Nijmegen. In 1987 she graduated in psycholinguistics, one of the specializations in linguistics. Her graduation year was spent at the Max-Planck Institut für Psycholinguistiek in Nijmegen, where she carried out research on the impact of context on ambiguities in text processing, reported in her essay. In that same year, she also became qualified to teach.

Since 1988 she is a member of the Department of Educational Development and Research of the University of Limburg in Maastricht, where she participated in the research project "Prior knowledge activation and text processing" of the Faculty of Medicine. In 1993 this assistant researcher (AIO) job was exchanged for a position as University Teacher (Universitair Docent) at the Faculty of Health Sciences of the University of Limburg. 